\title{
Unravelling epigenetic mechanisms of CAF-chemotherapy resistance in mammary carcinoma
}

\author{
Dissertation \\ for the award of the degree \\ "Doctor rerum naturalium" \\ of the Georg-August-Universität Göttingen \\ within the doctoral program Molecular Medicine
}

Submitted by

Iga Mieczkowska

born in Lublin, Poland

Göttingen, 2019 


\section{Thesis Supervisor:}

Dr. Florian Wegwitz

\section{Thesis Committee:}

Dr. Florian Wegwitz

Clinic for General, Visceral and Pediatric Surgery

University Medical Center Göttingen

Prof. Dr. Matthias Dobbelstein

Institute of Molecular Oncology

University Medical Center Göttingen

Dr. Nico Posnien

Department of Developmental Biology

University Medical Center Göttingen

\section{Members of the Examination Board:}

Prof. Dr. Heidi Hahn

Molecular Developmental Genetics

University of Göttingen

Prof. Dr. Frauke Alves

Molecular Imaging in Oncology

The Max Planck Institute of Experimental Medicine and UMG

Dr. Roland Dosch

Department of Developmental Biochemistry

University Medical Center Göttingen

Date of oral examination: 16.12 .2019 


\section{Affidavit}

I hereby declare that the $\mathrm{PhD}$ thesis entitled "Unravelling epigenetic mechanisms of CAF-chemotherapy resistance in mammary carcinoma" has been written independently and with no other sources and aids than quoted.

Iga Mieczkowska

November, 2019

Göttingen 


\section{Table of contents}

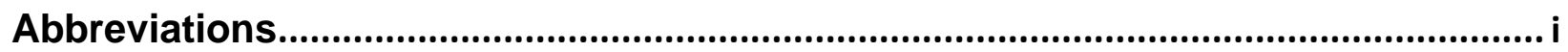

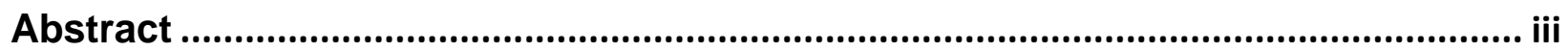

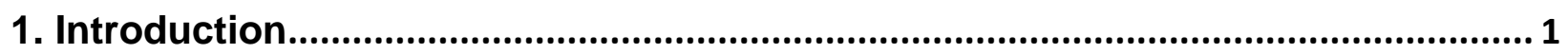

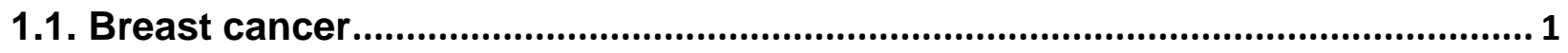

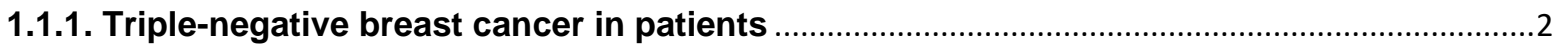

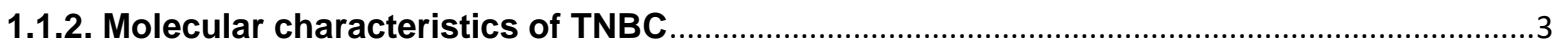

1.2. Chemotherapy resistance, EMT and stemness …............................................. 5

1.2.1. The WAP-T mouse model to study resistance to chemotherapy, in vivo and in vitro........9

1.3. Epigenetic modifications ........................................................................................... 11

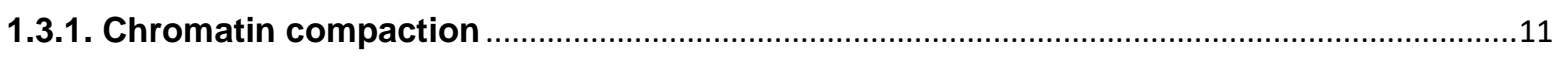

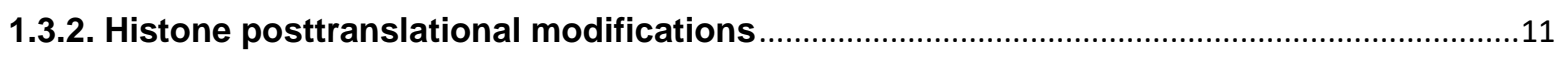

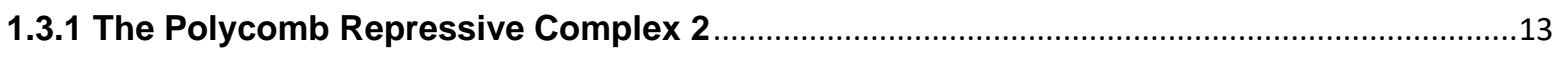

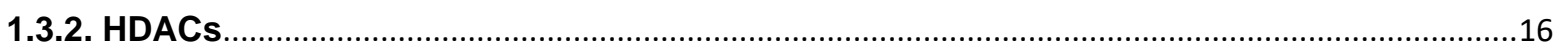

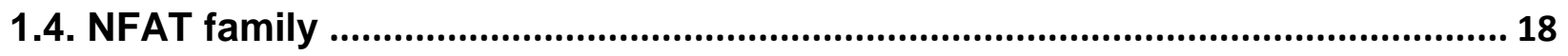

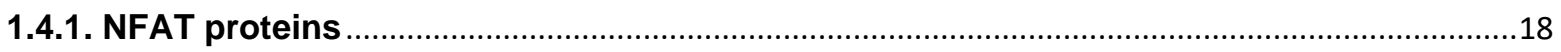

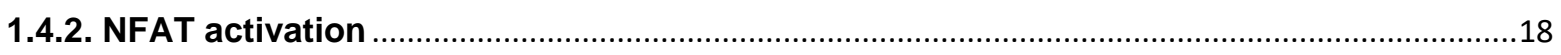

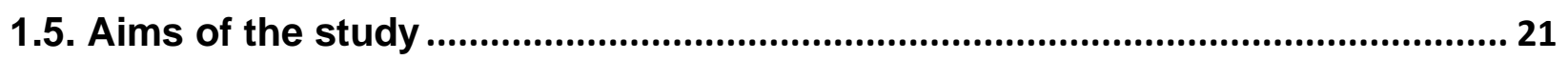

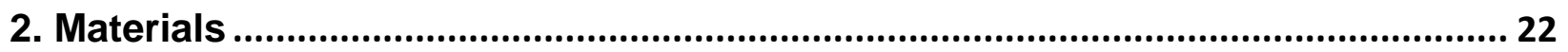

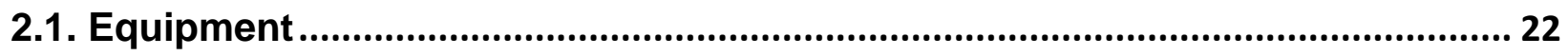

2.2. Consumable materials................................................................................................ 24

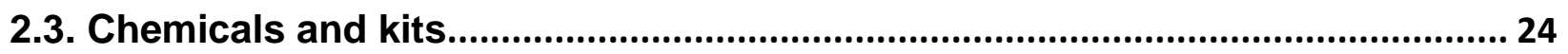

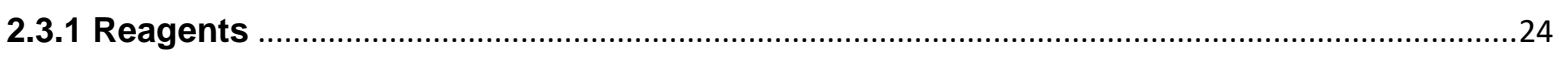

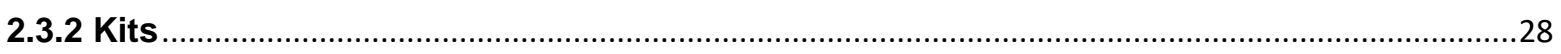

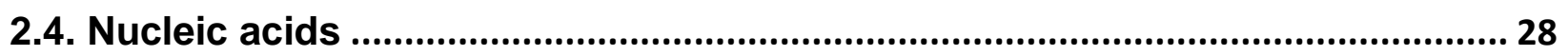

2.4.1. RT-PCR primers

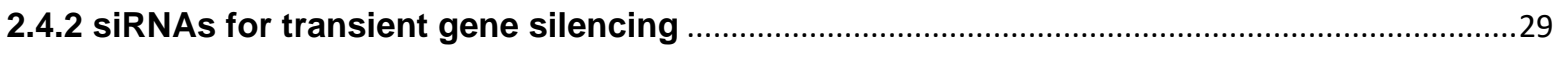

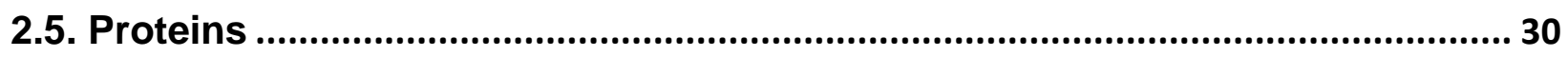

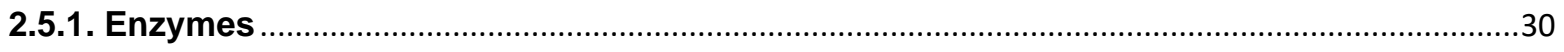

2.5.2. Antibodies

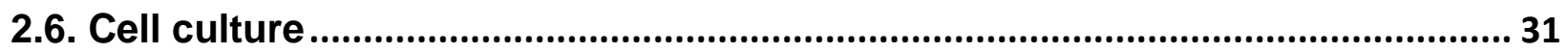

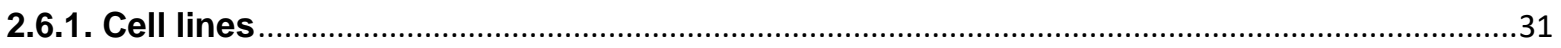




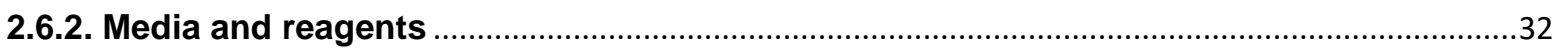

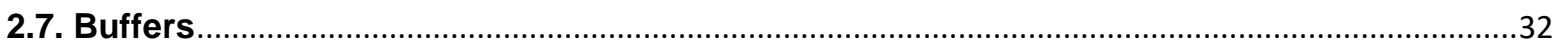

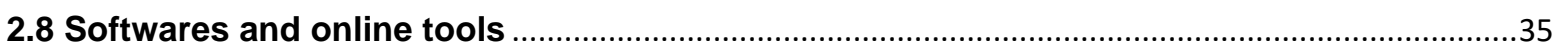

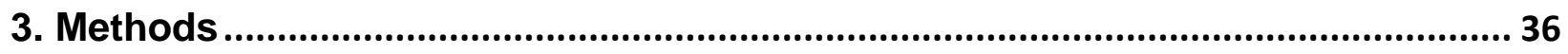

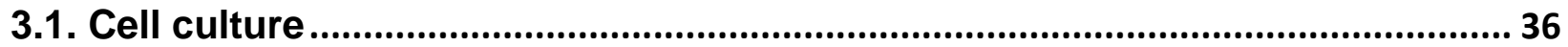

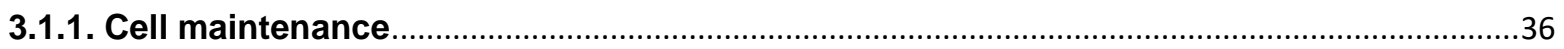

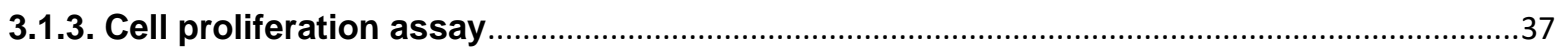

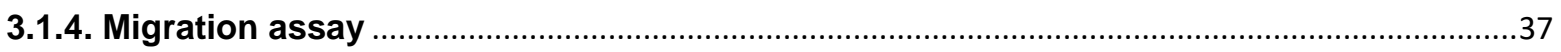

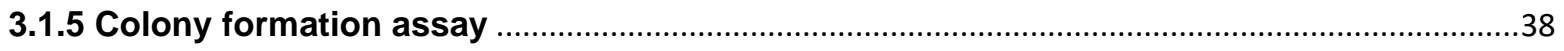

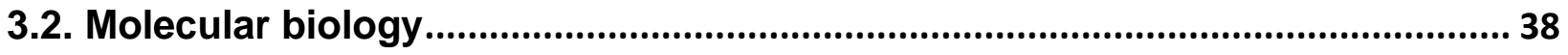

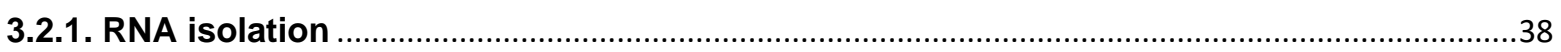

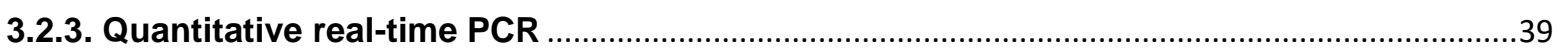

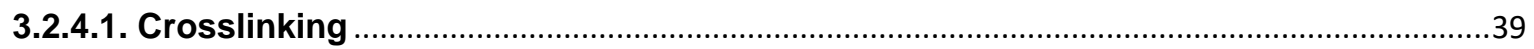

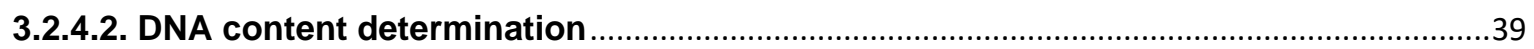

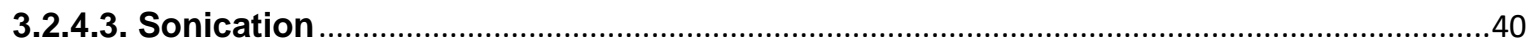

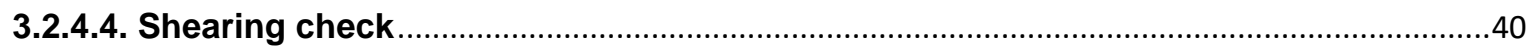

3.2.4.5. Pre-clearing and chromatin immunoprecipitation ...................................................40

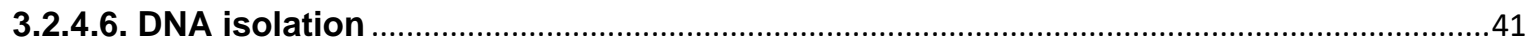

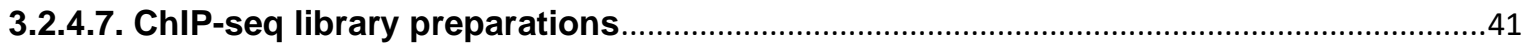

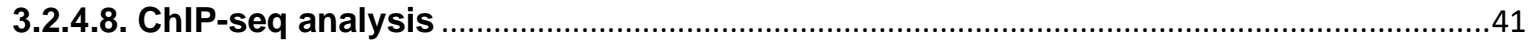

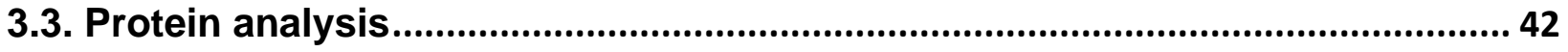

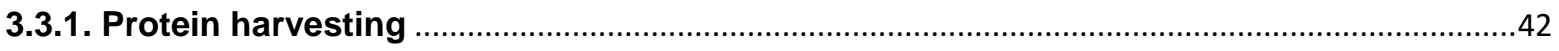

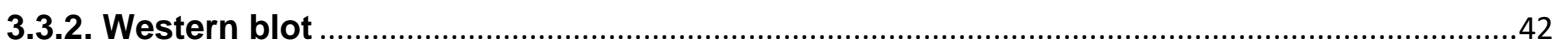

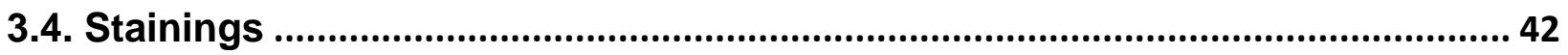

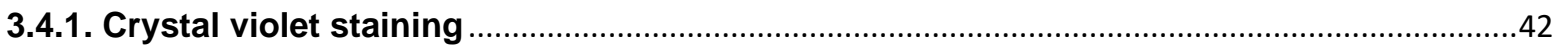

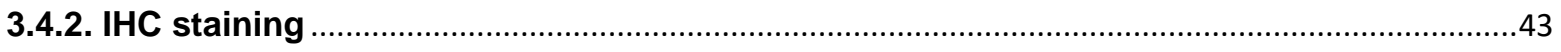

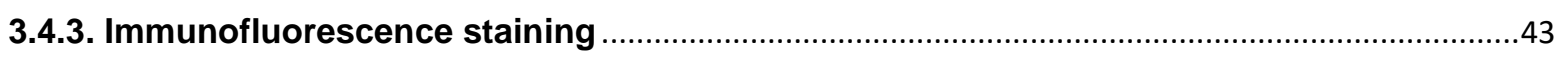

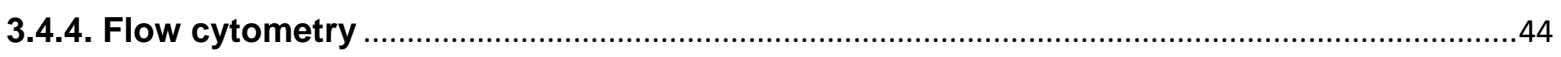

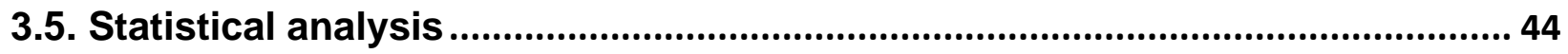

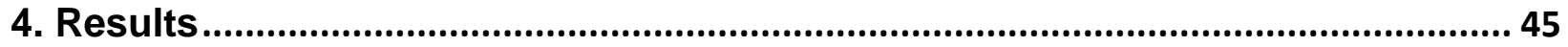

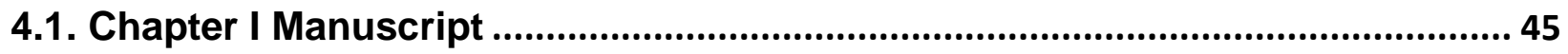

Reduction of PRC2/EZH2 activity can promote better survival of TNBC cancer

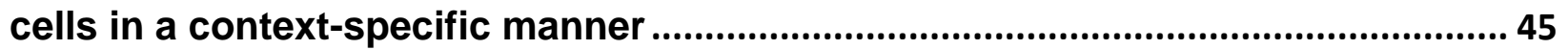

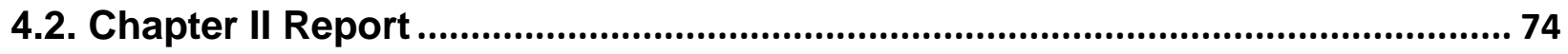


Therapeutical potential of HDACs in chemotherapy resistance in triple-negative

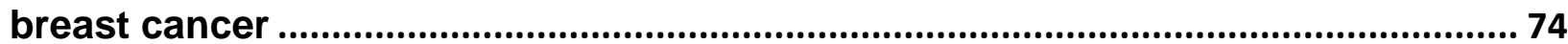

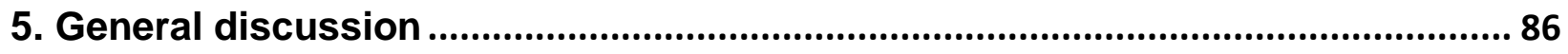

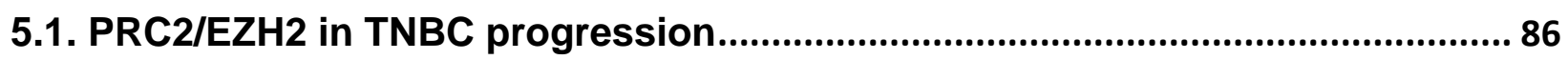

5.2. NFATC1 as a potential PRC2/EZH2-dependent driver of TNBC progression .. 88

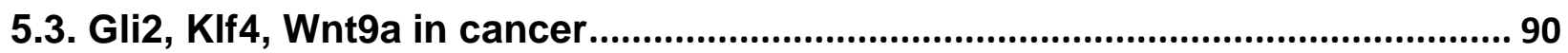

5.4. NFATc1 and HDAC inhibition in TNBC in the clinic .......................................... 91

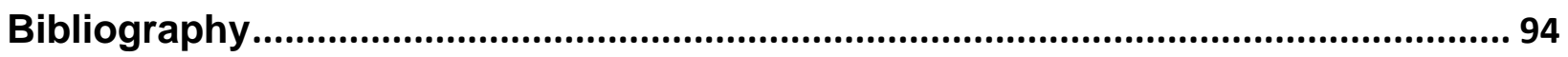




\section{Abbreviations}

\begin{tabular}{|c|c|}
\hline $5-\mathrm{FU}$ & 5-Fluorouracil \\
\hline BL1 & Basal-like 1 \\
\hline BL2 & Basal-like 2 \\
\hline BSA & Bovine serum albumin \\
\hline${ }^{\circ} \mathrm{C}$ & Degree Celsius \\
\hline $\mathrm{Ca}^{2+}$ & Calcium ions \\
\hline CAF & Cyclophosphamide, Doxorubicin (Adriamycin), 5-Fluorouracil \\
\hline Cat. no. & Catalog number \\
\hline cDNA & Complementary DNA \\
\hline ChIP & Chromatin immunoprecipitation \\
\hline ChIP-Seq & ChIP followed by high-throughput sequencing \\
\hline CsA & Cyclosporine A \\
\hline CSC & Cancer stem cell \\
\hline DAPI & 4',6-diamidino-2-phenylindole \\
\hline DEPC & Diethylpyrocarbonate \\
\hline DMEM & Dulbecco's modified eagle's medium \\
\hline DMSO & Dimethyl sulfoxide DNA deoxyribonucleic acid \\
\hline dNTP & Deoxyribonucleotide \\
\hline DTT & Dithiothreitol \\
\hline EDTA & Ethylenediaminetetraacetic acid \\
\hline EMT & Epithelial to mesenchymal transition \\
\hline $\mathrm{EtOH}$ & Ethanol \\
\hline $\mathrm{EZH} 2$ & Enhancer of zeste 2 \\
\hline FACS & Fluorescence-activated cell sorting \\
\hline FDA & Food and Drug Administration \\
\hline FDR & False discovery rate \\
\hline GSEA & Gene set enrichment analysis \\
\hline$H \& E$ & Hematoxylin and eosin \\
\hline $\mathrm{H} 3$ & Histone 3 \\
\hline HAT & Histone acetyltransferase \\
\hline HDAC & Histone deacetyltransferase \\
\hline HDACi & HDAC inhibitor \\
\hline H3K27ac & Histone 3 acetylated at position lysine 27 \\
\hline H3K27me3 & Histone 3 trimethylated at position lysine 27 \\
\hline IAA & lodacetamide \\
\hline
\end{tabular}




\begin{tabular}{|c|c|}
\hline $\mathrm{kDa}$ & kilo Dalton \\
\hline MEM & Minimum essential media \\
\hline ML & Mesenchymal-like \\
\hline MSL & Mesenchymal stem-like \\
\hline mRNA & messenger RNA \\
\hline NEM & N-ethylmaleimide \\
\hline NFAT & Nuclear factor of activated T-cells \\
\hline NGS & Next generation sequencing \\
\hline NP-40 & Nonidet P40 \\
\hline PBS & Phosphate buffered saline \\
\hline PBS-T & Phosphate buffered saline with tween-20 \\
\hline PcG & Polycomb group proteins \\
\hline PCR & Polymerase chain reaction \\
\hline PRC2 & Polycomb repressive complex 2 \\
\hline PTM & Post-translational modification \\
\hline RBBP7 & Retinoblastoma-binding protein 7 \\
\hline RNA & Ribonucleic acid \\
\hline RNA-seq & RNA sequencing \\
\hline Rpm & Rounds per minute \\
\hline RT & Room temperature \\
\hline RT-PCR/qPCR & Reverse transcription PCR \\
\hline SDS & Sodium dodecylsulfate \\
\hline SDS-PAGE & Sodium dodecylsulfate polyacrylamide gel electrophoresis \\
\hline siRNA & Small interfering ribonucleic acid \\
\hline SUZ12 & Suppressor of zeste 12 protein homolog \\
\hline siRNA & Small interfering RNA \\
\hline TEMED & Tetramethylethylenediamine \\
\hline TF & Transcription factor \\
\hline Tris & Tris(hydroxymethyl)aminomethane \\
\hline TSS & Transcriptional start site \\
\hline TNBC & Triple negative breast cancer \\
\hline VIM & Vimentin \\
\hline WAP & Whey acidic protein \\
\hline WB & Western blot \\
\hline Wnt & Wingless and Int- 1 \\
\hline WT & Wild type \\
\hline
\end{tabular}




\section{Abstract}

Triple negative breast cancer (TNBC) is one of the most challenging cancers to treat as, despite the initial response to chemotherapy, relapse occurs frequently. In this project, we focused on the mechanisms that allow TNBC cell survival under chemotherapy treatment. Phenotypic changes in TNBC cells such as gain of mesenchymal transition and stemness during genotoxic stress adaptation have been reported. Based on our data, CAF (Cyclophosphamide, Doxorubicin and 5Fluorouracil)-chemotherapy treatment in TNBC cells leads to downregulation of the Polycomb Repressive Complex containing the methyl transferase EZH2 (PRC2/EZH2) and upregulation of Histone Deacetylases (HDAC4, -7 and -8).

$E Z H 2$ is responsible for the gene silencing through $\mathrm{H} 3 \mathrm{~K} 27 \mathrm{me} 3$ catalysis and is known as an invasiveness marker. It appears that EZH2 can have also antitumorigenic function. We identified, among others, Nuclear factor of activated T cells (NFATC1), as a gene regulated by EZH2 loss upon chemotherapy treatment in TNBC cells, indicated epigenetic switch. NFATc1 showed H3K27me3 loss and H3K27ac gain upon chemotherapy, where H3K27ac works antagonistically to the repressive mark, H3K27me3. NFATc1 has been associated with drug resistance and its loss impairs proliferation, migration and mesenchymal properties in TNBC cells in vitro. Our results strongly indicate a negative regulation of EZH2 on NFATc1 expression, in vitro and in vivo. Additionally, in TNBC patient data, we observed that low EZH2 and high NFATc1 expression correlate with poor survival. Therefore, in patients showing low EZH2 expression in cancer, NFATc1 inhibition may represent an alternative treatment option.

Furthermore, our preliminary studies on HDACs suggest that HDAC8 supports TNBC invasiveness, affecting cell growth and modulating EMT. Interestingly, selective HDAC8 inhibition sensitizes TNBC cells to chemotherapy. This provides a potential mechanism linking epigenetic adaptation and cancer state during TNBC chemotherapy resistance.

In summary, this work demonstrates a previously unknown PRC2/EZH2 function in TNBC, where its downregulation contributes to NFAT pathway changes 
driving cancer progression. Hence, NFATc1 can be considered as potential PRC2/EZH2-dependent driver of TNBC invasiveness. Additionally, we identified HDAC8 and NFATC1 as an interesting targets for further investigation in potential antiTNBC therapeutic approach. 


\section{Introduction}

\subsection{Breast cancer}

According to the World Health Organization, breast cancer is the most common and most frequent diagnosed cancer among women causing a high cancer-related death rate (World Health Organisation, 2018). Breast cancer survival rates vary worldwide, where the highest cancer-related mortality occurs in developing countries (Ferlay et al., 2010). Despite scientific and diagnostic advancements leading to improved patient life quality, breast cancer remains a major cause of mortality (Lukong, 2017; World Health Organisation, 2018). Breast tumors are very complex and heterogeneous with a high diversity in gene expression patterns and pathological features. Based on the high-throughput transcriptomic analysis, breast tumors are classified into major molecular subtypes (Perou et al., 2000). These are Luminal A, Luminal B, human epidermal growth factor receptor 2 (HER2)-positive and triplenegative breast cancer (TNBC) (Figure 1) (Perou et al., 2000; Vallejos et al., 2010). The TNBCs account for 15-20\% of breast tumors. HER2-positive account for 10$15 \%$, Luminal B for $20 \%$ and Luminal A for $40 \%$ of breast cancer cases (MetzgerFilho et al., 2013). Breast cancer can be further categorized into subset, which based on three common immunohistochemical and targetable breast cancer biomarkers, the hormone receptors: estrogen receptor (ER), progesterone receptor (PR) and HER2.

The treatment of breast cancer varies according to the subtype. TNBC, as its name suggests, does not express any of these receptors. It is not responsive to target therapies against hormone receptors and HER2 receptor, therefore TNBC has the worst prognosis among breast cancer patients. The treatment of breast cancer varies according to the subtype. Local therapies include surgical resection and/or radiation (Matsen \& Neumayer, 2013). Patients with non-metastatic, hormone receptor-positive breast cancer (Luminal $A / B$ ) receive hormone therapy, also called endocrine therapy (Wong et al., 2012). To minimize the possibility of metastasis, surgery may be followed by adjuvant therapy, as additional to primary treatment (Dhankhar et al., 2010). Patients presenting metastasis are treated with chemotherapy alone or in combination 
with targeted therapies (Chacón López-Muñiz et al., 2019; Mayer \& Burstein, 2007). Patients with HER2-positive cancers can receive trastuzumab/lapatinib treatment to block HER2 in combination with chemotherapy. Overall, Luminal A/B and HER2positive present better treatment options. On the other hand, TNBC, as its name suggests, is not responsive to target therapies against hormone receptors and the HER2 receptor, therefore non-resectable TNBC patients are treated with chemotherapy (Prat, Pineda, et al., 2015).

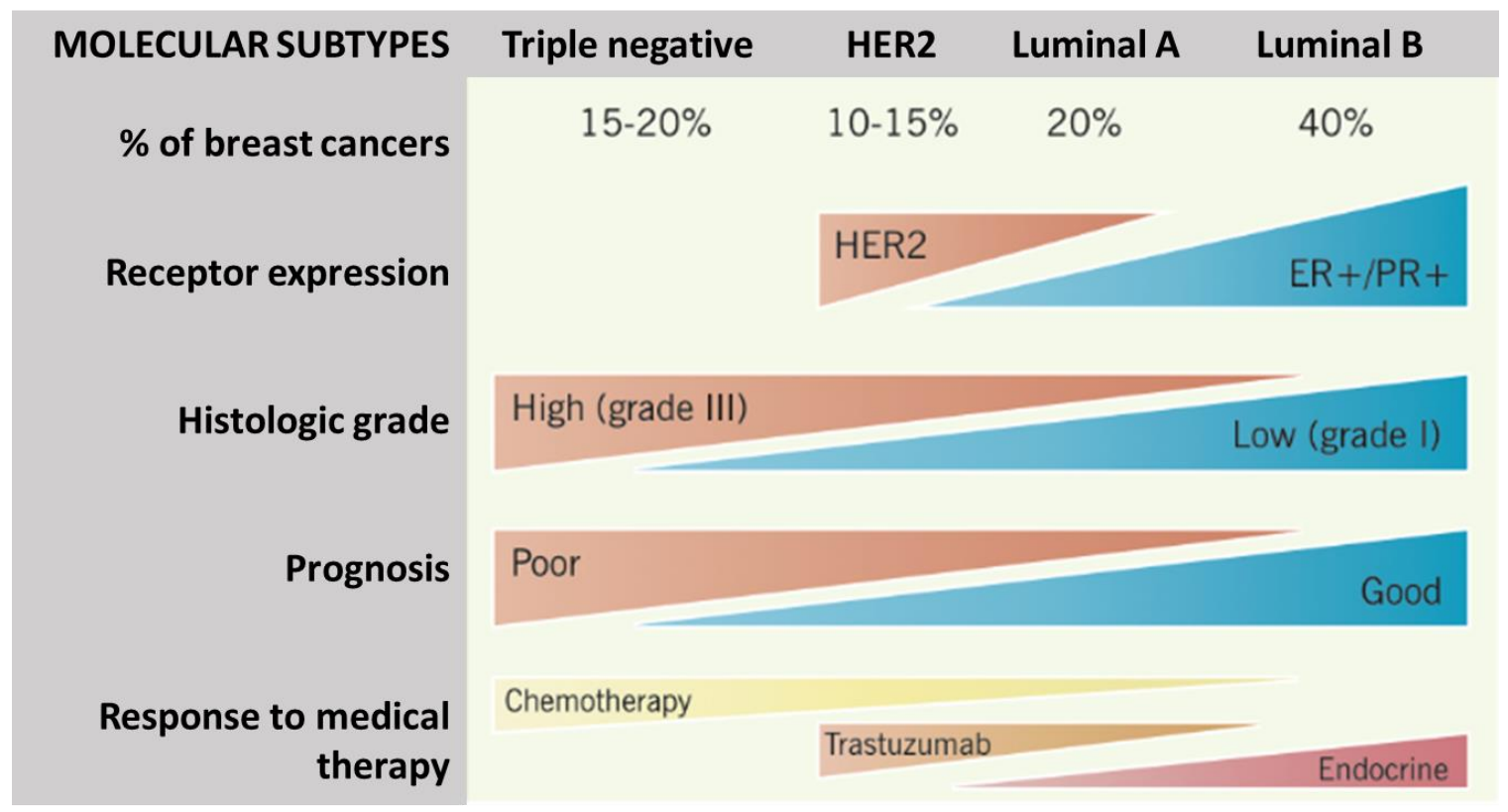

Figure 1: Breast cancer molecular subtypes presenting cancer occurrence, receptor expression, histologic grade (I-III), prognosis indicating chance of patient survival (poor- good) and therapy response indicating treatment option (chemotherapy, trastuzumab, endocrine therapy). (modified from Wong et al., 2012).

\subsubsection{Triple-negative breast cancer in patients}

TNBC shows high incidence in young ( $<40$ years old) African-American or Hispanic patients. These are often diagnosed at late cancer stage with a tendency to metastasize to lungs and brain (Morris et al., 2007; Sihto et al., 2011; Trivers et al., 2009). TNBC is the most aggressive breast cancer subtype correlating with poorer prognosis and a higher level of recurrence, mitotic rate, grade and tumor size than 
non-TNBC subtypes (Figures 1 and 2) (Gonçalves et al., 2018; Hirukawa et al., 2018). Additionally, it was shown that TNBC patients run a higher risk of having early metastasis after neoadjuvant chemotherapy than non-TNBC patients (Liedtke et al., 2008).

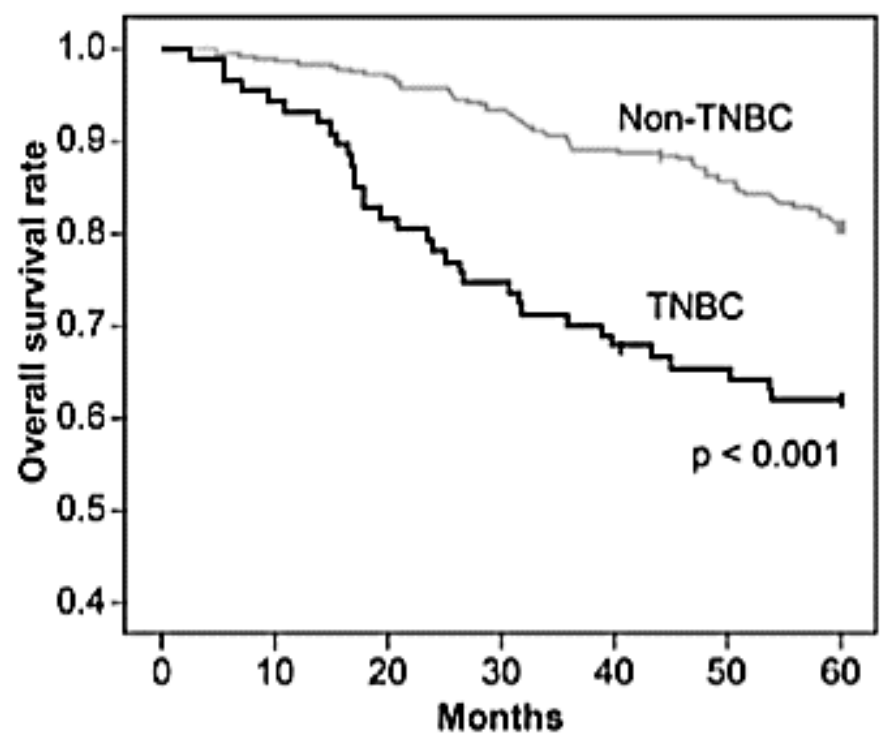

Figure 2: 5-year overall survival in TNBC and non-TNBC patients, $62.1 \%$ TNBC, $80.8 \%$ for nonTNBC cases ( $(P<0$.001) (Gonçalves et al., 2018).

\subsubsection{Molecular characteristics of TNBC}

TNBCs are defined by the lack of ER, PR and HER2, (Perou et al., 2000; Toft \& Cryns, 2010). Additionally, $80 \%$ of TNBCs harbor TP53 mutations, a percentage that is much less in other subtypes. Other features of this subtype include loss of RB1, $B R C A 1$ inactivation and high levels of $A K T 3$ and MYC (Koboldt et al., 2012). TNBC is a very heterogeneous disease and is classified into the following subgroups: basallike 1 and 2 (BL1 and BL2), immunomodulatory (IM), mesenchymal (M), mesenchymal-like (MSL) and luminal androgen receptor-positive (LAR) (Lehmann et al., 2011).

Figure 3 provides an overview about the most enriched pathway signature for each TNBC subtype with potential inhibitors. For instance, BL1 is associated with cell 
cycle, proliferation, DNA damage response pathways. Therefore, for instance the inhibitor of Poly (ADP-ribose) polymerase (PARP), which plays a role in DNA damage response, was one of the proposed drugs for this subgroup (Robson, 2011). It is known that $B R C A 1$ mutation, among others, associates with cancer progression and is highly correlated with the basal-like TNBC subgroup (Haffty et al., 2019). BRCA is responsible for the repair of double-stranded DNA breaks via homologous recombination (HR). BRCA1 mutations cause DNA repair errors leading to genomic instability and carcinogenesis (Buisson et al., 2010). It was shown that BRCA1deficient tumors seem to be sensitive to PARP inhibitors alone and in combination with cisplatin (Rottenberg et al., 2008). Using PARP inhibitors in BRCA-deficient cells leads to DNA damage and cancer cell death (Davar, 2012).

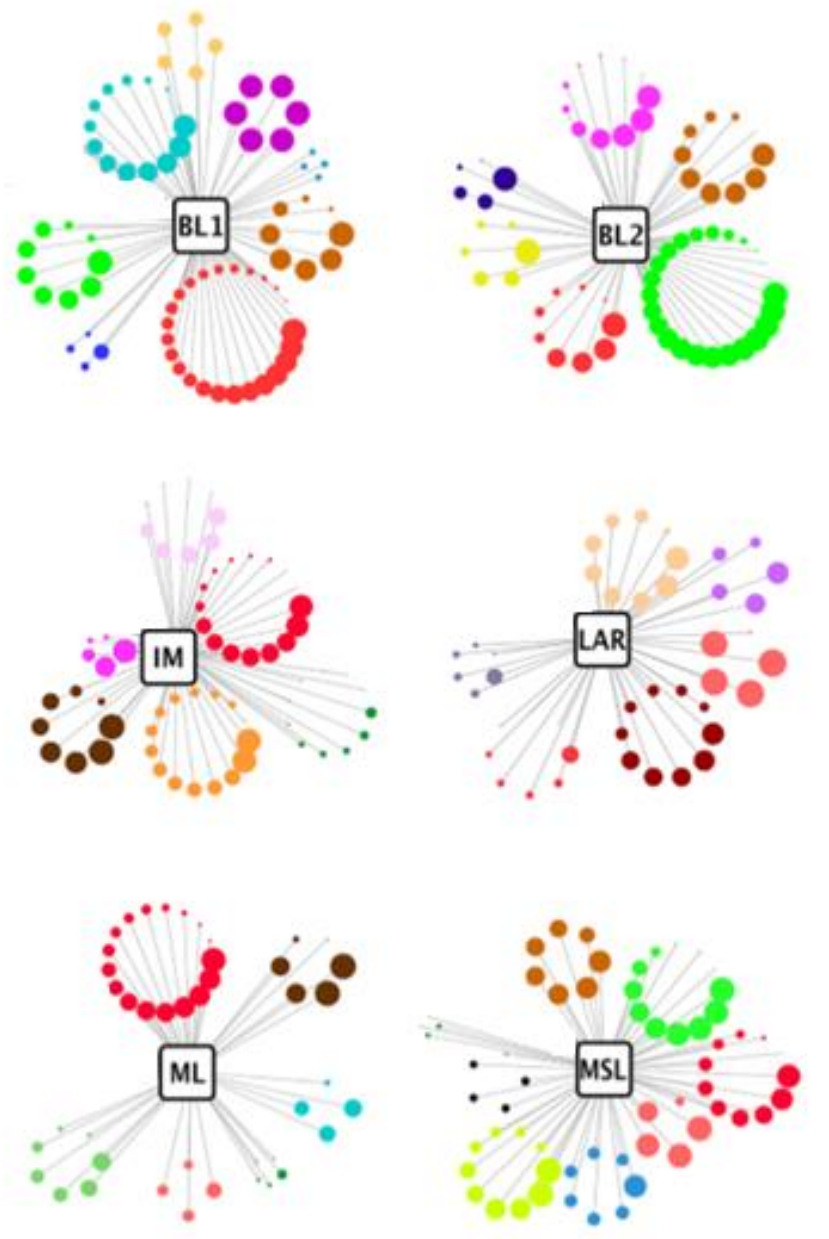

Adrenergic Antagonists

Alpha-2 Adrenergic Agonists

Androgen Receptor Antagonists

Angiotensin 2 Receptor Antagonists

Atypical Antipsychotics

Calcium Channel Antagonists

CDK Inhibitors

Cyclooxygenase Inhibitors

Endothelin Receptor Interactions

Histamine Receptor Interactions

HMG-CoA Reductase Inhibitors

Integrin Receptor Interactions

Interleukin Receptor Interactions

Janus Kinase Inhibitors

PARP Inhibitors

P13K Inhibitors

Potassium Channel Interactions

PPAR Activity

Protein Kinase Inhibitors

Receptor Tyrosine Kinase Inhibitors

TNF Receptor Blocking Activity

Tyrosine Kinase Inhibitors

VEGFR Inhibitors 
Figure 3: Drug classes in TNBC subtypes such as basal-like 1 and 2 (BL1 and BL2), immunomodulatory (IM), mesenchymal (M), mesenchymal-like (MSL) and luminal androgen receptor (LAR). Each dot represents one drug with its size corresponding to its rank position. Drug classes were derived into FDA New Drug Application (NDA) and GenEx-TNBC drug target profiles (Wathieu et al., 2017).

BL2 is associated with glycolysis, gluconeogenesis, angiogenesis and growth factor signaling pathways like EGF, NGF, MET and Wnt/ß-catenin (Shaw et al., 2010). The immunomodulatory subgroup displays a robust immune cell response through immune activation and immunological infiltration. The LAR subgroup partially shares the gene expression profile with TNBC, but also overlaps with HER-2 positive cancer (Wathieu et al., 2017). In the mesenchymal-like and mesenchymal stem-like TNBC subgroups, characteristic pathways correspond to the epithelial to mesenchymal transition (EMT), Wnt/ $\beta$-catenin, TGF- $\beta$ and PI3K/AKT/mTOR pathways (Massihnia et al., 2016). Taken together, TNBC subgroups are variable in gene expression signatures and respond differentially to particular chemotherapy agents dosage, combinations and treatment cycles, such that TNBC treatment remains largely unsuccessful (Oleg Gluz et al., 2008).

\subsection{Chemotherapy resistance, EMT and stemness}

In addition to surgery and radiation, chemotherapy is a common therapeutic option for many cancers. Many TNBC patients are better responders to chemotherapy in comparison to non-TNBC patients. It was reported that patients treated with adjuvant chemotherapy showed a $52 \%$ increase of survival than those treated with neoadjuvant chemotherapy (Echeverria et al., 2019). Consequently, apart from surgery, targeted therapy followed by chemotherapy can be a potential strategy to combat TNBC. The most common drugs used for chemotherapy treatments are intercalating agents, anthracyclines (epirubicin or doxorubicin), alkyling agents cross-linking within DNA resulting in mutations (cylophosphamide) and antimetabolites like the pyrimidine antagonist 5-fluorouracil (5-FU) or platinium-based agents (Kashiwagi et al., 2011; Sikov et al., 2015). TNBC resistance to chemotherapy is a major challenge in the clinic 
and there is a need to develop targeted treatments in addition to the conventional therapies described (William, 2008).

Resistance can emerge due to genetic alterations, where specific mutations can make cells insensitive to drug treatments. For example, loss of function TP53 allows cells to evade cell death and continue proliferation (Luqmani, 2005). Environment mediated-drug resistance also plays a pivotal role during treatment, fo example, cancer stem cells (CSCs) can scavenge chemotherapy by expressing major ABC transporters (Dean, Fojo, \& Bates, 2005).

Cancer stem cells are defined by three features such as their capacity to differentiate according to their hierarchical state, their self-renewal property to maintain stem cell population and their homeostasis between differentiation and selfrenewal, according to environmental stimuli (Dalerba, Cho, \& Clarke, 2007). The cancer stem cell model suggests that hierarchically organized cells display distinct tumorigenic and metastatic capacities generating tumor cell heterogeneity (Bonnet \& Dick, 1997; Shackleton et al., 2009). CSCs display stem-like features, slow growth rate and are involved in primary or acquired chemotherapy or radiotherapy resistance (Lajtha, 1967; Takebe et al., 2015). Additionally, aldehyde dehydrogenase (ALDH) activity is reported as a cell feature strongly correlated with self-renewal capacity (Marcato et al., 2011).

Recent studies on patient-derived xenografts (PDX) with TNBC showed the survival of residual cancer cells upon doxorubicin/cyclophosphamide treatments due to activated signaling pathways protecting cancer cells (Echeverria et al., 2019; Hutchinson et al., 2018). PDX models resistant to neoadjuvant chemotherapy treatment revealed that residual cancer cells displayed unique histological and transcriptomic features in comparison to untreated tumors. Due to the reversible nature of drug-tolerance, tumors can overcome chemotherapy without clonal selection (Echeverria et al., 2019). Cancer cells are able to adapt to a changing environment and stress insults by gene expression reprogramming. One way in which this can occur is that cells undergo epithelial to mesenchymal transition (EMT). This process is reversible and mesenchymal cells have the capacity to transition back to the epithelial state (MET), reflecting the plasticity of cancer cells (Figure 4). Mesenchymal 
cells with higher expression of VIMENTIN (VIM), SNAI1, N-CADHERIN $(N-C D H)$ have weak cell-to-cell contact and a greater invasion and migration potential. In contrast, epithelial cells show prominent expression of E-CADHERIN (E-CDH) or EPITHELIAL CELL ADHESION MOLECULE (EPCAM) (Berx et al., 1995). SNAl1, a zing-finger protein, is a transcriptional repressor that regulates $E-C D H$ in breast cancer, among others (Blanco et al., 2002; Paznekas et al., 1999). During carcinogenesis, E-CDH can be repressed via epigenetic modulation or a loss of function mutation (Hennig et al., 1995). EMT can be induced via HIF-1 $\alpha$, Wnt/ $\beta$-catenin, c-MET and TGF- $\beta$ (Bladt et al., 1995; Teng et al., 2014; Zhao et al., 2011). EMT was extensively described in the literature, showing that it is not sufficient for spontaneous breast cancer metastasis but is one of the mechanisms supporting cancer progression (Lou et al., 2008; Thiery, 2002). Interestingly, studies on sarcomatoid carcinoma consisting of mixed phenotypes of breast carcinoma and sarcoma, showed that two cancer cell types do not derive from stem cells of epithelial and mesenchymal origin but only from one epithelial cell. It could suggest that EMT was involved in cancer cell plasticity (Thiery, 2002). It was shown that the EMT program is associated with aggressiveness and metastasis in basal-like carcinoma (Ye et al., 2017). The EMT process can induce stem cell differentiation with reduced proliferation and the ability for therapeutic resistance. Standard chemotherapies lead to EMT activation where cancer cells can quickly adapt and overcome hostile environments promoting tumor cell survival and migration (Smith \& Bhowmick, 2016). 
A.

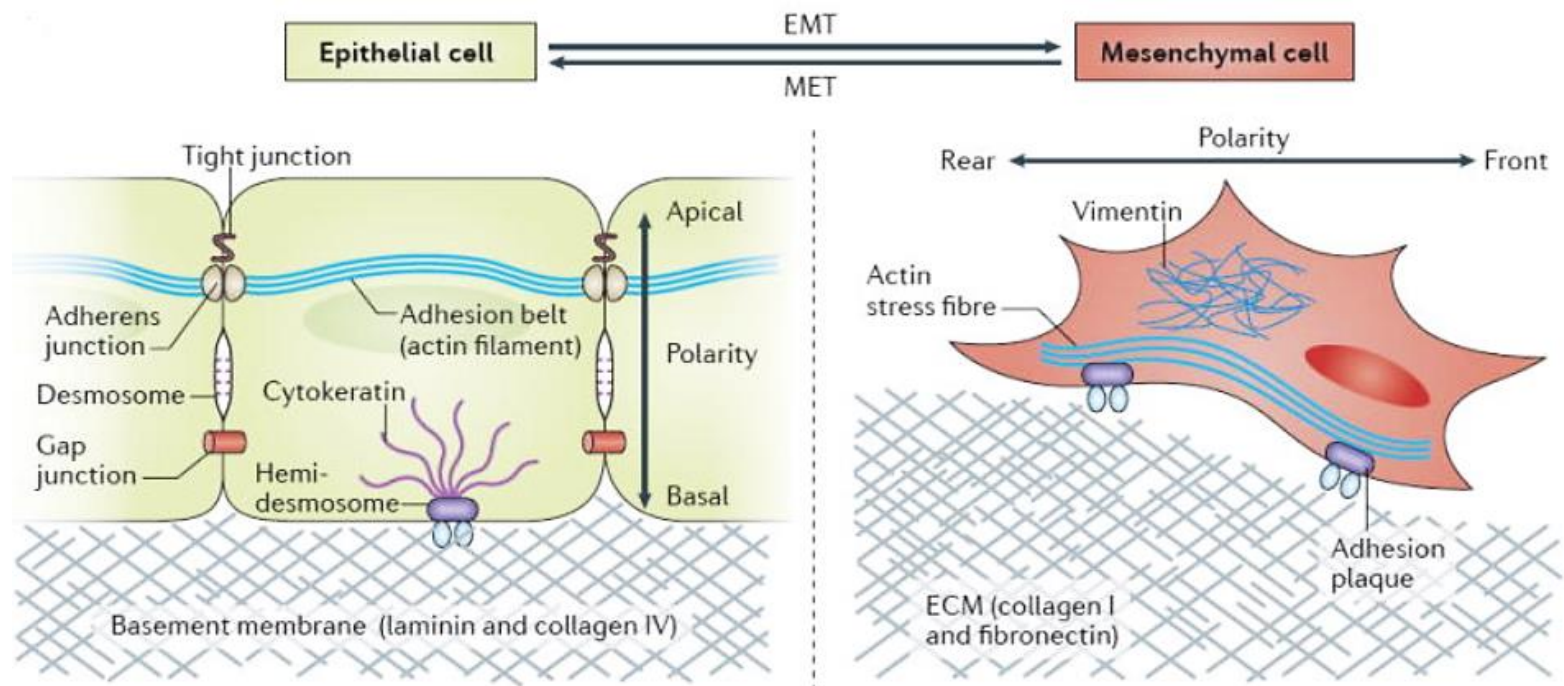

B.

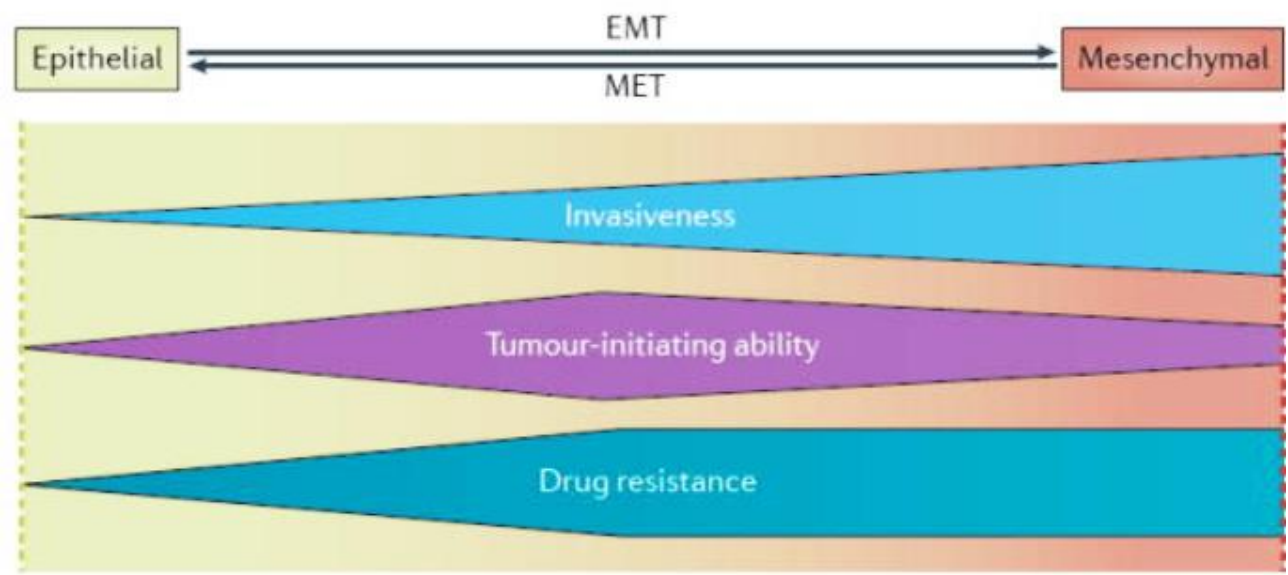

Figure 4: Epithelial to mesenchymal transition (EMT) and reversed mesenchymal to epithelial transition (MET). (A) Scheme of phenotypical and physiological changes during EMT including distinction in cell junction, polarity, cytoskeletal and protein rearrangement. (B) Invasive, tumorigenic and resistance potential within EMT/ MET process (Modified from Shibue \& Weinberg, 2017). 


\subsubsection{The WAP-T mouse model to study resistance to chemotherapy, in vivo and in vitro.}

The WAP-T mouse model was engineered to investigate mammary carcinoma cell properties, (Schulze-Garg et al., 2000). Immune-competent WAP-T mice mimic the clinical situation of basal-like TNBC subtypes in humans (Wegwitz et al., 2010). Mammary carcinogenesis in these mice is driven by the activation of a transgene, the simian virus 40 (SV40), under the control of the whey acidic protein (WAP) promoter. WAP is important for lactation in mammary epithelial cells, thus resulting in the expression of SV40 only in mammary cells (Hennighausen et al., 1990). SV40 large $T$ antigen and small $t$ antigen bind and suppress the tumor suppressors p53 and pRb inducing tumorigenic transformation (Kao et al., 1993).

To investigate the behavior of TNBC cells, a parental G-2 (pG-2) cell line was used. pG-2 (in the publications indicated as G-2 cells) are WAP-T mice-derived cells, displaying high heterogeneity, epithelial-mesenchymal plasticity (EMP) and stem-like features. Hence, this system is considered as a self-proliferating mammary carcinoma system (Maenz et al., 2015; Wegwitz et al., 2010). It was shown that treatment with selected cytotoxic agents like Cyclophosphamide, Doxorubicin (Adriamycin) and 5Fluorouracil (CAF) also kills the vast majority of these cells. However, a small remaining pool of cells representing cells with a more aggressive and invasive phenotype was observed (Figure 5). This is in accordance with previous findings, where tumors formed from the transplantation of $\mathrm{pG}-2$ cells into WAP-T mice, responded positively to CAF-chemotherapy. However, one round of CAF treatment was not sufficient to eliminate all cancer cells. Further analysis showed a great number of mesenchymal cells among disseminated tumor cells in comparison to untreated mice (Jannasch et al., 2015). Therefore, cancer cell plasticity represents a mechanism that can promote chemotherapy resistance. Interestingly, as described previously, cell plasticity can be mediated by reprogrammed gene transcription, which in part is regulated by epigenetic alterations. The tight regulation of epigenetic and transcriptional processes in the development of therapy resistance is essential and described in more detail in the next sections. 
A.
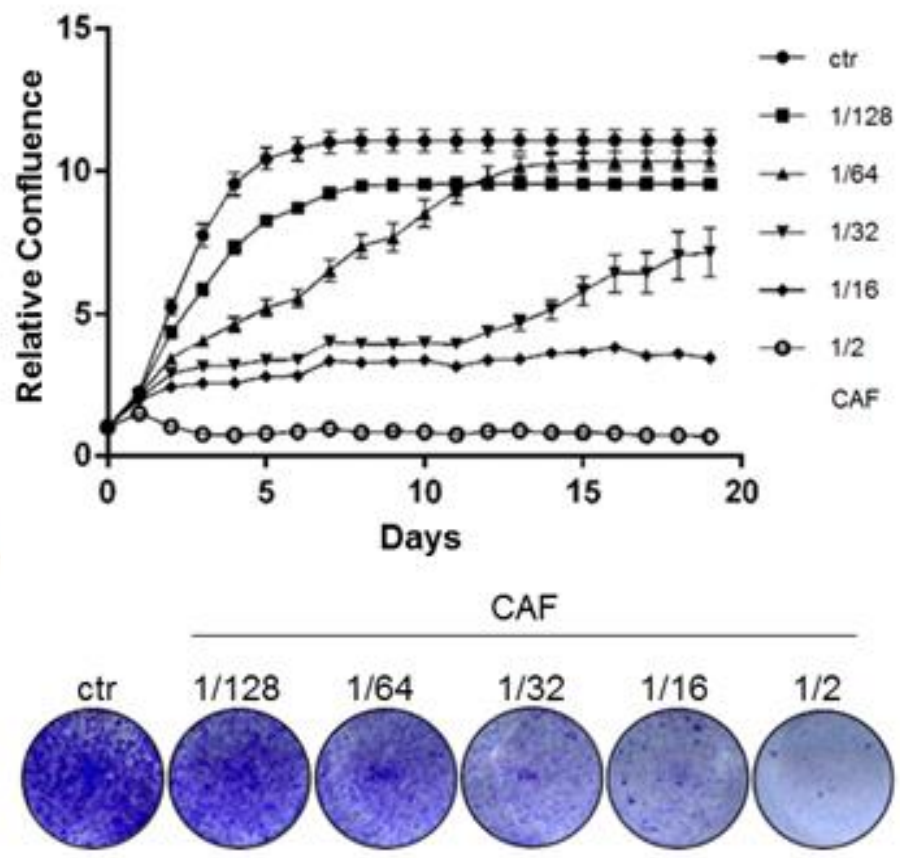

Figure 5: Optimization of CAF-chemotherapy treatment in pG-2 cells. (A) Proliferation curves of pG-2 cells treated with different chemotherapy concentrations: 1/2, 1/16, 1/32, 1/64, 1/128, where 1 represents concentrations $10 \mu \mathrm{g} / \mathrm{ml}$ Cyclophosphamide, $0.5 \mu \mathrm{g} / \mathrm{ml}$ Doxorubicin and $10 \mu \mathrm{g} / \mathrm{ml} 5$ Fluorouracil (CAF). Cells confluence was measured everyday using the Celigo ${ }^{\circledR}$ cell cytometer and normalized to results at day 0 . (B) Crystal violet staining of pG-2 cells at the end of the experiment followed by CAF-chemotherapy treatment (Schmidt, 2016). 


\subsection{Epigenetic modifications}

\subsubsection{Chromatin compaction}

Within the nucleus of eukaryotic cells, DNA is tightly coiled around histone octamers, composed of $\mathrm{H} 2 \mathrm{~A}, \mathrm{H} 2 \mathrm{~B}, \mathrm{H} 3$ and $\mathrm{H} 4$, resulting in a DNA-nuclear protein complex called chromatin (Arents, 1991; Luger, 1997). Chromatin can be organized in a more open structure, called euchromatin or highly condensed one, called heterochromatin (Figure 6) (Jenuwein \& Allis, 2001). Although high DNA compaction allows storing huge amount of genomic information in the nucleus, the access of factors regulating chromatin dynamics is limited due to the compaction. The structure and dynamics of chromatin is associated with gene expression regulation which determines the biological state of a given cell. Epigenetics involves mechanisms altering transcription of genes without changing the DNA sequence, regulating developmental paths initiating tissue-specific gene expression and therefore being crucial in cell fate determination (Reik, 2007; Waddington, 1957).

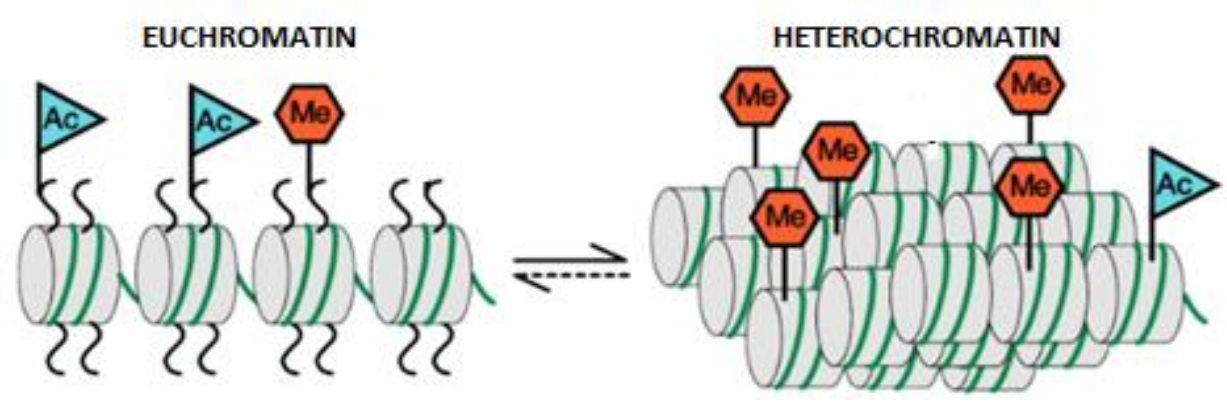

Figure 6: Model of euchromatin and heterochromatin with histone tail modifications; acetylation (Ac) and methylation $(\mathrm{Me})$ (modified from Jenuwein \& Allis, 2001).

\subsubsection{Histone posttranslational modifications}

Histone tails harbor several post-translational modifications (PTMs), such as acetylation, methylation, phosphorylation, sumoylation and ubiquitylation. The $\mathrm{N}$ terminal histone tails harbor a positive charge with a tendency to link to negatively charged DNA. Adding an acetyl group (acetylation) neutralizes the charge resulting in chromatin relaxation (Figure 5) allowing the euchromatin structure to become more accessible for transcription factors inducing transcription. The opposite effect occurs 
upon adding a methyl group leading to decreased recruitment of DNA binding proteins that promote transcription (Jenuwein \& Allis, 2001).

Proteins known as "writers", "erasers" and "readers" are responsible for adding, removing and recognizing particular post-translational histone modifications, respectively (Figure 7) (Audia \& Campbell, 2016). Epigenetic writers can be histone acetyltransferases (HATs) or histone methyltranferases (HMTs). Protein arginine methyltransferases (PRMTs) and protein lysine methyltransferases (KMTs) have the capacity to transfer a methyl group from the cofactor S-adenosylmethionine, called SAM to the arginine or lysine residues, respectively (Simõ-Riudalbas \& Esteller, 2015). Histone acetyltransferase can be reversed via histone deacetylases (HDACs). Histone methylation can be removed through the histone demethylases UTX-1 or JMJD3 (Agger et al., 2007). The bromodomain and extraterminal (BET) family including BRD2, BRD3, BRD4 and BRDT can bind to acetylated lysines on histone tails and further regulate the fate of the chromatin, playing roles in cell growth, chromatin remodeling and DNA damage (Dawson et al., 2011; Simõ-Riudalbas \& Esteller, 2015). Histone PTMs can occur on the global or locus-specific level. For instance, H3K27me mark can encompass distal enhancers, proximal promoters (enrichment around the transcription start site (TSS) and gene bodies (Figure 7) (Young et al., 2011). A particular epigenetic mark occupancy can modulate transcription, via fine-tuning RNA polymerase II (RNAPII) recruitment to the promoter and/or enhancer region. In this case, $\mathrm{H} 3 \mathrm{~K} 27 \mathrm{me} 3$ on enhancers and promoters limits RNAPII progression hindering transcription (Chopra et al., 2011).

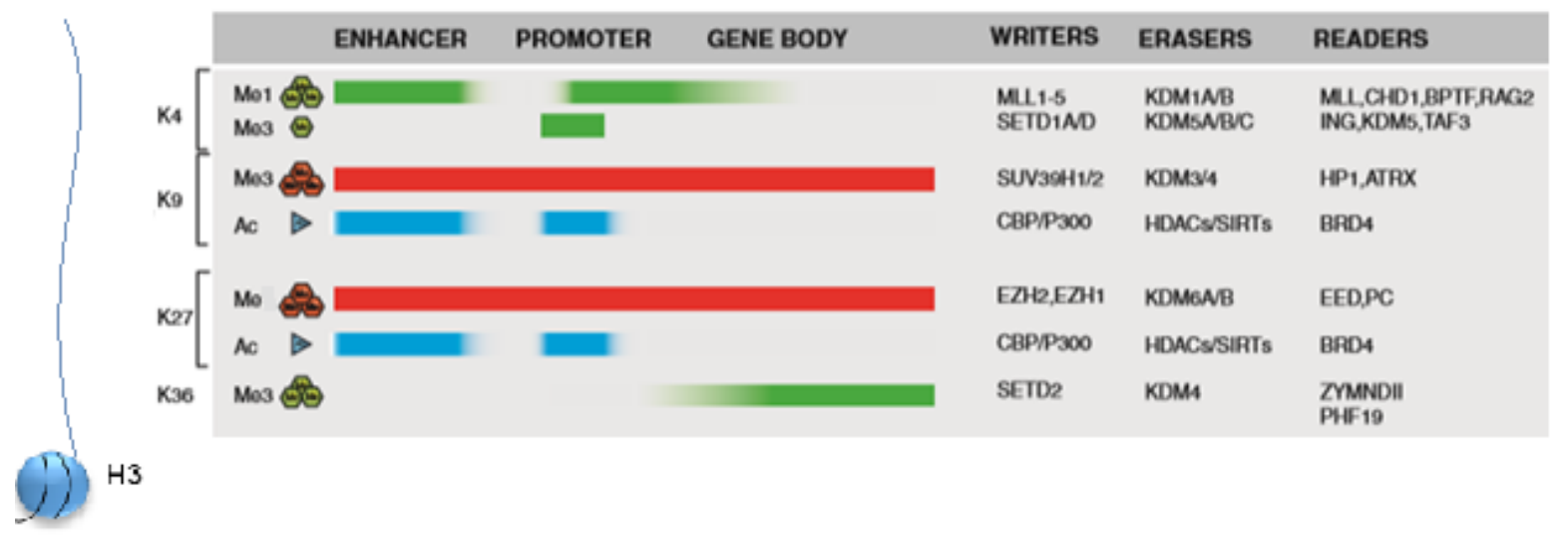


Figure 7: Histone writers, erasers, and readers. Posttranslational modifications (PTMs) on Histone 3 $(\mathrm{H} 3)$ tail (left) along the gene loci (enhancer, promoter, gene body). Histone marks are indicated in green (methylation) and blue (acetylation) for active genes and in red shading for silent genes. Examples of writers, erasers and readers are indicated on the right side (modified from Audia \& Campbell, 2016).

The most studied epigenetic marks with opposing functions are trimethylation of histone 3 at the position of lysine 27 (H3K27me3) and its acetylation (H3K27ac) (Figure 8). H3K27 methylation, in general, is responsible for maintaining the repression of specific target genes, while acetylation promotes chromatin relaxation and transcription activation. Therefore, $\mathrm{H} 3 \mathrm{~K} 27 \mathrm{me} 3$ and H3K27ac are considered as a repressive and active mark, respectively. These chromatin modifications are involved in the molecular regulation of gene expression patterns and can determine cell fate (Katoh et al., 2018).

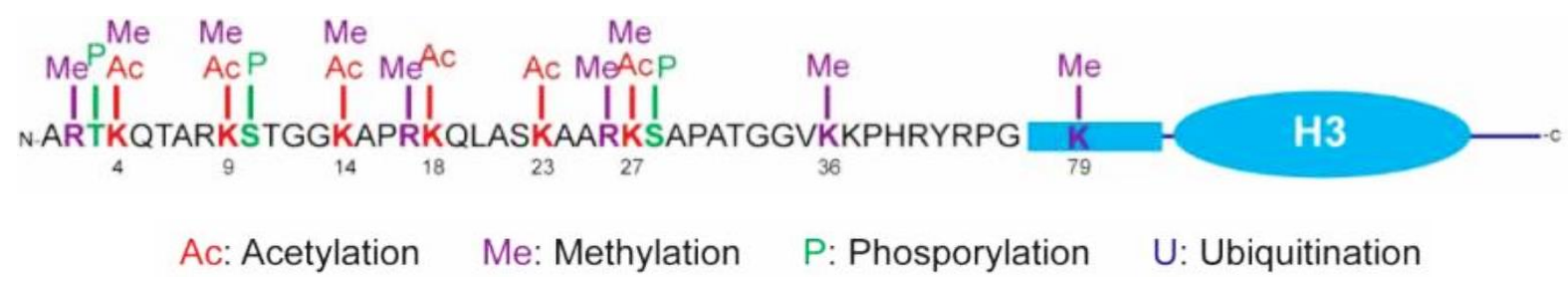

Figure 8: Chromatin modifications on histone 3 tail. Acetylation (Ac), methylation (Me), phosphorylation $(\mathrm{P})$ and ubiquitination $(\mathrm{U})$ occur mostly in $\mathrm{N}$-terminal $\mathrm{K}$ and $\mathrm{R}$ rich tails (modified from Parsons, 2014).

\subsubsection{The Polycomb Repressive Complex 2}

One of the most important transcriptional modulators are polycomb group proteins ( $P c G$ ), playing a role in cell proliferation and differentiation (Antonysamy et al., 2013). Two main PcG complexes have been described, Polycomb repressive complex 1 (PRC1) and 2 (PRC2). PRC2 represses genes by catalyzing the trimethylation of histone 3 at lysine 27. The Chromobox protein (CBX) subunit of PRC1 recognizes H3K27me3 and another member of the PRC1, E3 ubiquitin-protein ligase (RING1A/B), ubiquitinates histone $H 2 A$ on $K 119$ residue (H2AK119) maintaining a repressed chromatin state (H2AK119ub) (Di Croce \& Helin, 2013). 
PRC2 takes part in many biological processes, from differentiation to stem-cell plasticity, including repression of numerous developmental regulators in embryonic stem cells (Boyer et al., 2006; Raphaël Margueron \& Reinberg, 2011). Apart from EZH1/2, PRC2 consists of Suppressor of zeste 12 protein homolog (SUZ12), Embryonic ectoderm development protein (EED) and Retinoblastoma-binding protein (RbAp46/48, also known as RBBP4/7). Enhancer of zeste 1 and $2(E Z H 1 / 2)$ are protein homologs forming similar PRC2 complexes (PRC1/EZH1, and PRC2/EZH2). However, the PRC2/EZH1 complex seems to have a lower enzymatic activity and, in contrast to $\mathrm{EZH} 2$, loss of $\mathrm{EZH} 1$ does not result in a global loss of di- or trimethylation of H3K27 (H3K27me2/3). Previous studies have shown that both EZH1 and EZH2 repress transcription in vitro (Raphael Margueron et al., 2008). Additionally, Ezh1 is ubiquitously expressed, whereas Ezh2 expression is tightly associated with proliferating cells (Raphael Margueron et al., 2008). EZH2 is an essential PRC2 member, requiring other PRC2 binding partners SUZ12, RBBP7 and EED to be active (Denisenko et al., 1998; Liu et al., 2015; Pasini et al., 2004).

The canonical PRC2/EZH2 pathway leads to gene silencing that can be diverse among organisms and tissues. (Cao \& Zhang, 2004; Gan et al., 2018). PRC2 proteins silence target genes upon binding to specific genomic regions called polycomb responsive elements (PREs) (Liu et al., 2015). EZH2 also methylates non-histone proteins such as STAT3, GATA4, RORa, resulting in their regulation (Figure 9) (He et al., 2012; E. Kim et al., 2013; Lee et al., 2012). Interestingly, apart from its transcriptional repressive function, in certain context, EZH2 can also display transactivating functions positively influencing NF-KB, Wnt/ $\beta$-catenin and ERa-driven signaling, in a PRC2-indendependent manner (Jung, H. et al., 2013; K. H. Kim \& Roberts, 2016). EZH2 can cooperate with the androgen receptor (AR) and the estrogen receptor $(E R \alpha)$, promoting prostate and breast cancer progression, respectively (Shi et al., 2007; Xu et al., 2012). 


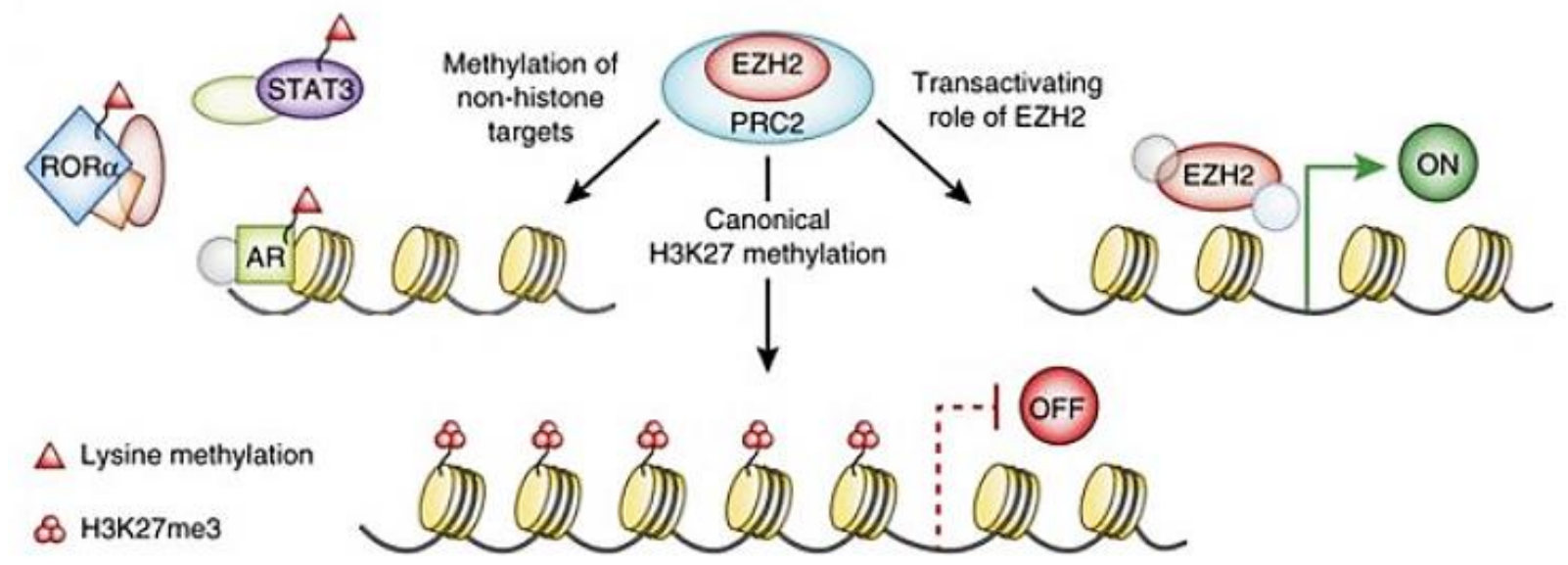

Figure 9: $\mathrm{EZH} 2$ transcriptional activity. $\mathrm{PRC2} / \mathrm{EZH} 2$ gene silencing via $\mathrm{H} 3 \mathrm{~K} 27 \mathrm{me} 3, \mathrm{EZH} 2$ methylation of non-histone proteins and PRC2-independent EZH2 transcriptional activation (modified from K. H. Kim \& Roberts, 2016).

Aberrant PRC2/EZH2 activity is known to have oncogenic activity in a number of tumors (C. Chang \& Hung, 2011). EZH2 is very frequently overexpressed in diverse tumors entities like pancreatic, prostate, breast and bladder cancer ( $\mathrm{Y}$. Chen et al., 2010; Kleer et al., 2003; Raman et al., 2005; Varambally et al., 2002). A few studies suggest a pro-tumorigenic EZH2 activity in TNBC. Chien et al. suggest that EZH2 promotes TNBC invasiveness via TIMP2/MMP-2 and -9 regulation (Chien et al., 2018) Another study on MDA-MB-231, a TNBC cell line, revealed that co-treatment with EZH2 and HDAC inhibitors leads to tumor cell death.

However, a growing number of studies reported an anti-tumorigenic function of $\mathrm{PRC2/EZH2}$. In medulloblastoma, it was shown that the inactivation of EZH2 can promotes Myc-driven cancer (Vo et al., 2017). It was also reported that PRC2 loss promotes chemoresistance with reduction of apoptosis in $\mathrm{T}$ cell acute lymphoblastic leukemia (Ariës et al., 2018). The role of EZH2 remains elusive in distinct TNBC subgroups. EZH2 could be an oncogene or tumor suppressor in TNBC and can regulate key genes in a context-dependent manner in different cancers. 


\subsubsection{HDACs}

H3K27 can be methylated as mentioned before but also acetylated through histone acetyltransferases (HATs) transfer an acetyl group from a donor molecule, acetyl coenzyme $A$ (acetyl-CoA), to the $\varepsilon$-amino group of lysine residues of the substrate (Vogelauer et al., 2012). Histone acetylation can be reversed by histone deacetylases (HDACs). HDACs can be classified into class I (HDAC1, -2, -3, -8) class Ila (HDAC4, -5, -7, -9), class Ilb (HDAC6, -10), class III (sirtuins) and class IV (HDAC11) (Gregoretti et al., 2004).

Aberrant HDAC gene expression is frequently associated with cancer development (reviewed in Y. Li \& Seto, 2016). HDACs are overexpressed in various tumors including gastric, breast and prostate cancer, which have higher levels of HDAC1 and colorectal cancer, which upregulate HDAC2 and HDAC3 (Kawai et al., 2003; Mariadason, 2008; Weichert et al., 2008). HDAC inhibitors (HDACi) represent interesting molecules to reverse cancer progression, as they can change the acetylation status of histone and non-histone proteins (Figure 10). HDAC inhibition can alter gene expression inducing apoptosis through Fas, DR5, TRAIL and caspases (Rosato, 2005). These inhibitors have also been shown to impose cell cycle arrest at G0/G1 or G2/M checkpoints and to reduce angiogenesis and metastasis. HDACs are able to target cytoplasmic proteins, such as heat shock protein 90 (HSP90), DNA repair factor Ku70, $\alpha$-tubulin and $\beta$-catenin (Krämer et al., 2014; Ma et al., 2009). Additionally, it was shown that inhibition of HDACs can suppress TGF- $\beta 1$-induced EMT and chemotherapy resistance through SMAD4 inhibition (T. Sakamoto et al., 2016). 


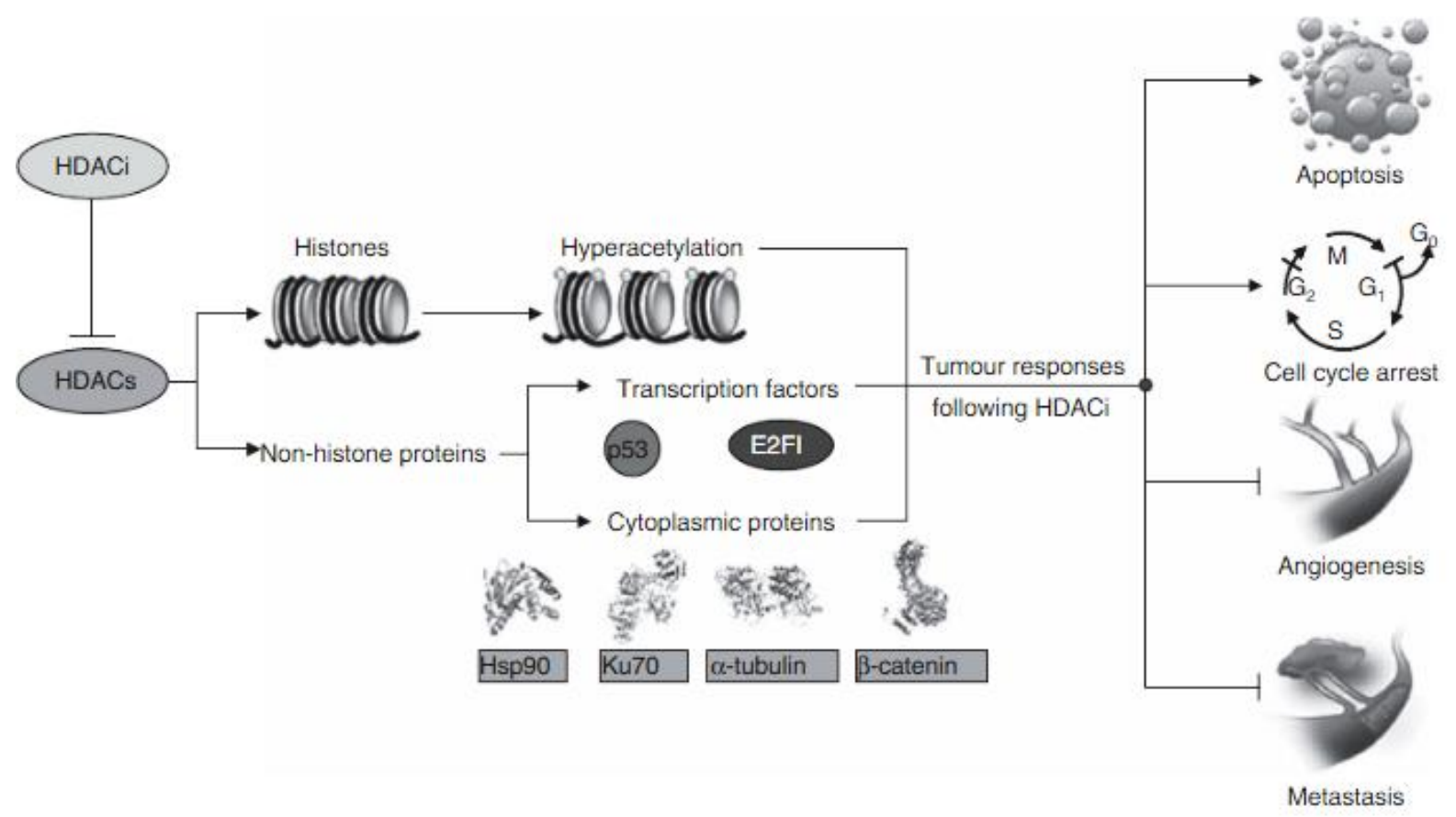

Figure 10: Cellular effects of HDAC inhibitors in cancer. Inhibition of chromatin repression via HDACi can affect apoptotic, cell cycle, angiogenic and metastatic changes in cancer cells through abrogation of acetylation/ deacetylation balance in chromatin and non-histone proteins (modified from Ma et al., 2009)

One of the most well-known and FDA approved drugs in T-cell lymphoma are Vorinostat (SAHA) and Panobinostat (LBH589) which are pan-HDAC inhibitors, targeting class I and II HDACs (Marks \& Breslow, 2007; Ververis et al., 2013). In breast cancer, HDAC inhibitors can be used as monotherapy or in combination with other drugs, such as lapatinib (anti-HER2), tamoxifen (anti-ER), olaparib (PARPi) or cisplatin (Hasan et al., 2018; Min et al., 2015; Solomon et al., 2015).

Despite many promising in vitro and in vivo studies, results from preclinical trials failed when HDACi was used as a single agent (Slingerland et al., 2014). HDAC inhibitors have shown promising results in combination with other anticancer therapies. According to studies on MDA-MB-231, a TNBC cell line, SAHA treatment alone promotes EMT transition via HDAC8/FOXA1 signaling (Oehme et al., 2009). Instead, SAHA in combination with IR radiation or cisplatin can decrease tumor growth, induce apoptosis and cell cycle arrest (Chiu et al., 2013). Furthermore, the 
use of a combination of DNA methyltransferases (DNMT) and HDAC inhibitors to dampen TNBC aggressiveness was proposed recently (Su et al., 2018).

\subsection{NFAT family}

The Nuclear factor of activated T cells (NFAT) family is composed of transcription factors, which induce gene expression during immune responses (Rao et al., 1997). The first report on NFATc2 described it as a DNA-binding protein binding to the interleukin-2 (IL-2) promoter in T cells (Shaw et al., 1988). It is known that NFATs exist also in other immunoregulatory cells such as B cells, NK cells, macrophages, etc. (Rao et al., 1997). Recently, particular research efforts have been made to unravel the function of NFATs factors in cancer (Robbs et al., 2008).

\subsubsection{NFAT proteins}

The NFAT family consists of five members: NFAT1 (NFATC2), NFAT2 (Nfatc1), NFAT3 (NFATc4), NFAT4 (NFATc3) and NFAT5 (Rao et al., 1997) (Table I 1). NFAT1-4 have a regulatory region called NFAT homology region (NHR) including the transactivation domain (TAD) and a calcineurin docking site (CDS). Moreover, there is a highly conserved DNA-binding domain called Rel-homology domain (RHD) and a carboxy-terminal domain (Luo et al., 1996; Müller \& Rao, 2010). Within conserved serine-rich regions (SRR), there are 14 docking sites for phosphorylation of NFATs by CK1, GSK3 and DYRK (Hogan et al., 2003). The N-terminal domain contains two nuclear localization sequences (NLS1, NLS2) and one nucleus export signal (NES) controlling subcellular transport and localization (Beals et al., 1997).

\subsubsection{NFAT activation}

Except for NFAT5 that is activated by osmotic stress, NFAT1-4 are calcium responsive proteins. (Luo et al., 1996). NFATs become activated after cytosolic $\mathrm{Ca}^{2+}$ influx, which is a consequence of $G$ protein coupled receptor signaling or ER stress (Prakriya et al., 2006). Upon physiological stimulation and decrease of $\mathrm{Ca}^{2+}$ levels in the $E R$, the plasma membrane-located CRAC channel is activated to restore calcium ions to normal levels, thus increasing cytosolic calcium levels. Cytoplasmic calcium 
binds to and induces conformational changes of the protein calmodulin. The latter can then bind and activate calcineurin, a calcium-dependent phosphatase that is able to dephosphorylate NFATs, rendering them active. The phosphorylation status of NFATs determines their activity and intracellular localization where highly phosphorylated NFATs remain in the cytoplasm and calcineurin-dependent dephosphorylated NFATs are translocated to the nucleus (Hogan et al., 2003).

Table 1. NFATc1 family members and their regulation (adapted from Macian, 2005).

\begin{tabular}{llll}
$\begin{array}{l}\text { NFAT family } \\
\text { member }\end{array}$ & Alternative names & $\begin{array}{l}\text { Regulation } \\
\text { NFAT1 }\end{array}$ NFATc2 and NFATp & $\begin{array}{l}\text { Expression in the } \\
\text { immune system }\end{array}$ \\
NFAT2 & NFATc1 and NFATc & $\begin{array}{l}\text { Calcineurin } \\
\text { Calcium- }\end{array}$ & Yes \\
calcineurin & Yes \\
NFAT3 & NFATc4 & $\begin{array}{l}\text { Calcium- } \\
\text { calcineurin }\end{array}$ & No \\
NFAT4 & NFATc3 and NFATx & $\begin{array}{l}\text { Calcium- } \\
\text { calcineurin }\end{array}$ & Yes \\
\hline NFAT5 & TonEBP and OREBP & Osmotic stress & Yes \\
\hline NFAT, nuclear factor of activated T cells; TonEBP, tonicity-responsive enhancer-binding protein.
\end{tabular}

The NFAT signaling pathway is reported to be related to cancer progression (Mancini \& Toker, 2009). The calcineurin inhibitor, cyclosporine A (CsA) can reduce NFAT activity by binding to cyclophlin $A$ and creating a complex that prevents calcineurin from interacting with calmodulin even in the presence of calcium (Flanagan et al., 1991). Studies demonstrate that cyclosporine $A$ is well tolerated by patients in advanced solid malignancies in I/IB clinical trials. They present CsA as calcineurin inhibitor suppressing $\mathrm{Wnt} / \mathrm{Ca}^{2+} / \mathrm{NFAT}$ pathway (Krishnamurthy et al., 2018). CsA could also be combined with chemotherapy treatment of drug-resistant solid tumors (Stiff \& Marrow, 1995). Another, more selective NFAT inhibitor is VIVIT and its therapeutic potential was observed in cardiovascular disorders and chronic lymphocytic leukemia resulting in the reduction of disease progression (Le Roy et al., 2012; Yu et al., 2007). The catalytic inhibition of sarcoplasmic reticulum ATPase (SERCA2) by thapsigargin makes it possible to investigate the role of NFATs in vitro and in vivo by promoting the activation of the latter (Prasad \& Inesi, 2009). 


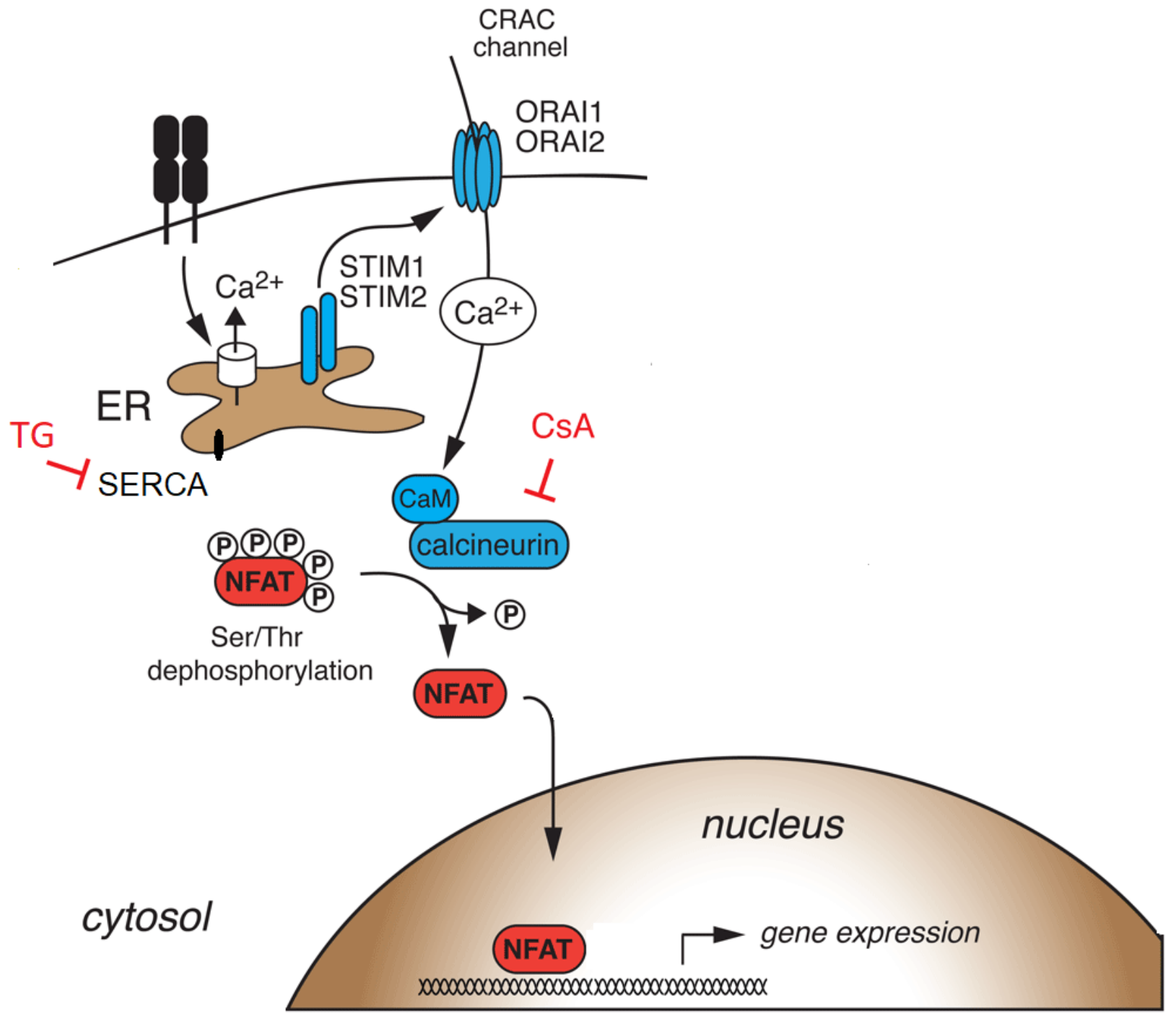

Figure 11: NFAT activation. Calcium/calcineurin, in the presence of $\mathrm{Ca}^{2+}$ ions, can dephosphorylate NFATs resulting in their translocation to the nucleus. CsA and Thapsigargin inhibit and activate NFATs, respectively (modified from Vaeth \& Feske, 2018). 


\subsection{Aims of the study}

Triple-negative breast cancer (TNBC) is the most challenging breast cancer subtype in the clinic routine. Due to lack of hormone therapy, conventional chemotherapy remains the mainstay in TNBC treatment. However, some cancer cells can be insusceptible to cytotoxic drugs leading to cancer recurrence. Remaining tumor cells adapt to hostile conditions by changing their gene expression and phenotype. Shedding light on the mechanisms driving chemotherapy-resistance is of utmost importance to understand TNBC progression.

Epigenetic regulatory pathways are fast and therefore likely to be implicated in overcoming chemotherapy that is to be associated with switches in gene expression pattern and acquisition of more aggressive features. The initial aim of this study was therefore to identify altered epigenetic mechanisms upon survival of tumor cells to cytotoxic drugs. Genome wide transcriptome (mRNA-seq) and histone mark occupancy (ChIP-seq) analyses were designed to identify up- or down-regulated epigenetic factors and to unravel the potential gene expression programs under control of these epigenetic processes. After subsequent validation of the findings via IHC staining on tumor material and via publically available databases mining, we aimed to assess the potential of interfering with the newly identified epigenetic mechanisms of survival to chemotherapy via in vitro functional assays.

Taken together, the present study ultimately aimed to provide new insights about mechanisms underlying chemotherapy resistance that could subsequently serve as a basis for the development of more efficient anti-TNBC therapeutic strategies. 


\section{Materials}

\subsection{Equipment}

2100 Bioanalyzer

Agarose gel chamber
Agilent Technology, Santa Clara, USA Harnischmacher Labortechnik, Kassel, Germany

Balance $440-35 \mathrm{~N}$

Biological Safety Cabinet "Safe 2020"

Celigo ${ }^{\circledR}$ S Cell Imaging CytometerNexcelom Bioscience LLC, Lawrence, USA

Centrifuge (Megafuge 1.OR)

Centrifuge (5417R)

Centrifuge (Heraeus Fresco 21)

Counting chamber (Neubauer)

DynaMag-2

DynaMag-96 Side Magnet

Eclipse TS100

Electrophoresis \& Electrotransfer Unit

Freezer $-150{ }^{\circ} \mathrm{C}$ (MDF-C2156VAN)

Freezer $-20^{\circ} \mathrm{C}$

Freezer $-80{ }^{\circ} \mathrm{C}$ "Hera freeze"

Gel iX Imager

HERAcell 150i CO2 Incubator

Imager Western Blot

Inverse Microscope "Axiovert 40 CFL"

Isotemp ${ }^{\circledR}$ water bath

Magnet stirrer "MR3001"

Microcentrifuge C1413-VWR230

Microscope Axio Scope.A1 with
Thermo Fisher Scientific, Waltham, USA

Eppendorf, Hamburg, Germany

Thermo Fisher Scientific, Waltham, USA

Brand GmbH \& Co. KG, Wertheim, Germany

LifeTechnology, Carlsbad, USA

LifeTechnology, Carlsbad, USA

Nikon, Tokio, Japan

GE Healthcare Europe $\mathrm{GmbH}$, München, Germany

Panasonic, Kadoma, Japan

Liebherr $\mathrm{GmbH}$, Biberach, Germany

Thermo Fisher Scientific, Waltham, USA

Intas Science Imaging $\mathrm{GmbH}$, Göttingen, Germany

Thermo Fisher Scientific, Waltham, USA

Bio-Rad Laboratories, Hercules, USA

Carl Zeiss Microlmaging GmbH, Göttingen, Germany

Thermo Fisher Scientific, Waltham, USA

Heidolph GmbH \& Co. KG, Schwabach, Germany

VWR, Radnor, USA

Carl Zeiss Microlmaging GmbH, Göttingen, 
an AxioCam MRc

Microscope Axiovert 100

Microwave

Mini Trans-Blot ${ }^{\mathrm{TM}}$ Cell

Mini-PROTEAN Tetra Cell

Mr. Frosty ${ }^{\circledR}$ Cryo Freezing Container

Nano Drop ${ }^{\circledR}$ ND-1000

Optical Reaction Module CFX96 ${ }^{\mathrm{TM}}$

$\mathrm{pH}$ meter inoLab ${ }^{\circledR}$

Pipette Aid ${ }^{\circledR}$ portable XP

Pipettes "Research" Series

Power supply Power Pack P25T

PowerPac ${ }^{\mathrm{TM}}$ Basic Power Supply

PowerPac ${ }^{\mathrm{TM}}$ HC Power Supply

Qubit $^{\circledR}$ 2.0 Fluorometer

Photo Scanner Epson V700

Refrigerator

Shaker "Rocky"

Test Tube Rotator

Thermal Cycler $\mathrm{T} 100^{\mathrm{TM}}$

ThermoMixer $\mathrm{C}$

Vortex-Genie 2T
Germany

Carl Zeiss Microlmaging GmbH, Göttingen, Germany

Clatronic International $\mathrm{GmbH}$, Kempen, Germany

Bio-Rad Laboratories, Hercules, USA

Bio-Rad Laboratories, Hercules, USA

Thermo Fisher Scientific, Waltham, USA

Peqlab Biotechnology $\mathrm{GmbH}$, Erlangen, Germany

Bio-Rad Laboratories, Hercules, USA

WTW GmbH, Weilheim, Germany

Drummond Scientific Co., Broomall, USA

Eppendorf, Hamburg, Germany

Biometra $\mathrm{GmbH}$, Göttingen, Germany

Bio-Rad Laboratories, Hercules, USA

Bio-Rad Laboratories, Hercules, USA

Invitrogen $\mathrm{GmbH}$, Karlsruhe, Germany

Seiko Epson, Suwa, Japan

Liebherr $\mathrm{GmbH}$, Biberach, Germany

Schütt Labortechnik GmbH, Göttingen, Germany

Schütt Labortechnik GmbH, Göttingen, Germany

Bio-Rad Laboratories, Hercules, USA

Eppendorf, Wessling-Berzdorf, Germany

Electro Scientific Industr. Inc., Portland, USA 


\subsection{Consumable materials}

96-well Multiplate ${ }^{\circledR}$ PCR plate, white Cell scraper $(16 \mathrm{~cm}, 25 \mathrm{~cm})$

Cell culture dishes $(10 \mathrm{~cm}, 14.5 \mathrm{~cm})$

Cellstar 6-,12-well cell culture plates

Costar 24- well cell culture plates
Bio-Rad Laboratories, Hercules, USA Sarstedt AG \& Co., Nümbrecht, Germany Greiner Bio-One $\mathrm{GmbH}$, Frickenhausen, Germany

Greiner Bio-One GmbH, Frickenhausen, Germany

Corning Incorporated, New York, USA

\subsection{Chemicals and kits}

\subsubsection{Reagents}

Acetic acid

Carl Roth GmbH \& Co. KG, Karlsruhe, Germany

Agarose

Albumin Fraction $\mathrm{V}(\mathrm{BSA})$

GeneOn $\mathrm{GmbH}$, Ludwigshafen, Germany Carl Roth GmbH \& Co. KG, Karlsruhe, Germany

Ammonium persulfate (APS)

Carl Roth GmbH \& Co. KG, Karlsruhe, Germany

Ammonium sulfate (NH4)2SO4

Carl Roth GmbH \& Co. KG, Karlsruhe, Germany

Aprotinin Carl Roth GmbH \& Co. KG, Karlsruhe, Germany

Bromophenol blue Sigma-Aldrich Co., St. Louis, USA

Calcium Chloride $\left(\mathrm{CaCl}_{2}\right)$ Carl Roth GmbH \& Co. KG, Karlsruhe, Germany

Colorless co-precipitant Ambion, Altham, USA

Co-precipitant Pink Bioline, Luckenwalde, Germany

Crystal violet Merck Millipore, Darmstadt, Germany Cyclophosphamide Pharmacy, University Medicine Göttingen 
Cyclosporine A

Diethylpyrocarbonate (DEPC)

Dimethyl sulfoxide (DMSO)

di-Sodium hydrogen phosphate

DMEM

dNTPs

Doxorubicin

Ethanol absolute

EDTA

EPZ-6438 (S7128)

Fetal Bovine Serum (FBS)

Fluorouracil

Formaldehyde

Gene RulerTM DNA-Ladder

Glycerol

Glycine

Guava EasyCyte plus

USA

HD Green ${ }^{\circledR}$ DNA stain

Hydrochloric acid $(\mathrm{HCl})$

lodacetamide
Biozol, Eching, Germany

Carl Roth GmbH \& Co. KG, Karlsruhe, Germany

Carl Roth GmbH \& Co. KG, Karlsruhe, Germany

Carl Roth GmbH \& Co. KG, Karlsruhe, Germany

GlutaMax GIBCO, Invitrogen $\mathrm{GmbH}$, Darmstadt, Germany Jena Bioscience GmbH, Jena, Germany Pharmacy, University Medicine Göttingen Merck Millipore, Darmstadt, Germany Carl Roth GmbH \& Co. KG, Karlsruhe, Germany

Selleckchem, Houston, USA

Thermo Scientific HyClone, Logan, USA

Pharmacy, University Medicine Göttingen

Sigma-Aldrich Co., St. Louis, USA

Fermentas $\mathrm{GmbH}$, St. Leon-Rot, Germany

Carl Roth GmbH \& Co. KG,

Karlsruhe,Germany

Carl Roth GmbH \& Co. KG, Karlsruhe, Germany

Guava Technologies Inc., San Francisco,

Intas Science Imaging $\mathrm{GmbH}$, Göttingen, Germany

Carl Roth GmbH \& Co. KG, Karlsruhe, Germany

Sigma-Aldrich Co., St. Louis, USA 


\begin{tabular}{|c|c|}
\hline Isopropanol & $\begin{array}{l}\text { Carl Roth GmbH \& Co. KG, Karlsruhe, } \\
\text { Germany }\end{array}$ \\
\hline Leupeptin & $\begin{array}{l}\text { Carl Roth GmbH \& Co. KG, Karlsruhe, } \\
\text { Germany }\end{array}$ \\
\hline Lithium chloride (LiCl), 8M & Sigma-Aldrich Co., St. Louis, USA \\
\hline Magnesium chloride (MgCl2) & $\begin{array}{l}\text { Carl Roth GmbH \& Co. KG, Karlsruhe, } \\
\text { Germany }\end{array}$ \\
\hline Methanol & $\begin{array}{l}\text { Carl Roth GmbH \& Co. KG, Karlsruhe, } \\
\text { Germany }\end{array}$ \\
\hline N-ethylmaleimide (NEM) & Sigma-Aldrich Co., St. Louis, USA \\
\hline Nickel chloride (NiCl2) & Sigma-Aldrich Co., St. Louis, USA \\
\hline $\mathrm{N}$-Lauryl sarcosine & Sigma-Aldrich Co., St. Louis, USA \\
\hline Nonidet ${ }^{\mathrm{TM}} \mathrm{P} 40$ (NP-40) & Sigma-Aldrich Co., St. Louis, USA \\
\hline Opti-MEM & $\begin{array}{l}\text { GIBCO Invitrogen GmbH, Darmstadt, } \\
\text { Germany }\end{array}$ \\
\hline \multicolumn{2}{|c|}{ PageRuler ${ }^{\mathrm{TM}}$ Prestained Protein Ladder Fermentas $\mathrm{GmbH}$, St. Leon-Rot, Germany } \\
\hline PBS tablets & $\begin{array}{l}\text { GIBCO Invitrogen GmbH, Darmstadt } \\
\text { Germany }\end{array}$ \\
\hline Pefabloc SC Protease Inhibitor & $\begin{array}{l}\text { Carl Roth GmbH \& Co. KG, Karlsruhe, } \\
\text { Germany }\end{array}$ \\
\hline Penicillin-Streptomycin solution & Sigma-Aldrich Co., St. Louis, USA \\
\hline PMSF & $\begin{array}{l}\text { Calbiochem, VWR International GmbH, } \\
\text { Darmstadt, Germany }\end{array}$ \\
\hline Proteinase $\mathrm{K}$ & Invitrogen $\mathrm{GmbH}$, Karlsruhe , Germany \\
\hline Protein-A Sepharose & CL-4B GE Healthcare, Uppsala, Sweden \\
\hline Reverse Transcriptase (M-MuLV) & New England Biolabs, Frankfurt am Main, \\
\hline \multicolumn{2}{|l|}{ Germany } \\
\hline Roti Phenol/Chloroform/Isoamyl & $\begin{array}{l}\text { Carl Roth GmbH \& Co. KG, Karlsruhe, } \\
\text { Germany }\end{array}$ \\
\hline RNase A & $\begin{array}{l}\text { Macherey-Nagel GmbH \& Co. KG, Düren, } \\
\text { Germany }\end{array}$ \\
\hline
\end{tabular}


Rotiphorese Gel 30

Rotipuran Chloroform

RPMI 1640

Sepharose ${ }^{\mathrm{TM}} \mathrm{CL}-4 \mathrm{~B}$

Skim milk powder

Sodium acetate

Sodium azide

Sodium chloride $(\mathrm{NaCl})$

Sodium deoxycholate

Sodium dodecylsulfate (SDS)

Sodium fluoride $(\mathrm{NaF})$

Sodium hydroxide $(\mathrm{NaOH})$

$\beta$-Glycerolphosphate (BGP)

SYBR Green

Taq DNA Polymerase

TEMED

TMP195

TMP269

Tris

Triton X-100

TRIzol Reagent
Carl Roth GmbH \& Co. KG, Karlsruhe, Germany

Carl Roth GmbH \& Co. KG, Karlsruhe, Germany

Life Technologies, Carlsbad, USA

GE Healthcare, Uppsala, Sweden

Carl Roth GmbH \& Co. KG, Karlsruhe, Germany

Carl Roth GmbH \& Co. KG, Karlsruhe, Germany

AppliChem GmbH, Darmstadt, Germany

Carl Roth GmbH \& Co. KG, Karlsruhe, Germany

AppliChem GmbH, Darmstadt, Germany

Carl Roth $\mathrm{GmbH}$ \& Co. KG, Karlsruhe, Germany

AppliChem GmbH, Darmstadt, Germany

Carl Roth GmbH \& Co. KG, Karlsruhe, Germany

Sigma-Aldrich Co., St. Louis, USA

Roche Diagnostics $\mathrm{GmbH}$, Mannheim, Germany

Prime Tech, Minsk, Belarus

Carl Roth GmbH \& Co. KG, Karlsruhe, Germany

Biomol, Hamburg, Germany

Biomol, Hamburg, Germany

Carl Roth GmbH \& Co. KG, Karlsruhe, Germany

AppliChem GmbH, Darmstadt, Germany

Invitrogen $\mathrm{GmbH}$, Karlsruhe, Germany 
Trypsin-EDTA (0.05\%)

Tween-20

$\alpha, \alpha-T r e h a l o s e ~ D i h y d r a t e$
GIBCO, Invitrogen GmbH, Darmstadt, Germany

Carl Roth GmbH \& Co. KG, Karlsruhe, Germany

AppliChem GmbH, Darmstadt, Germany

\subsubsection{Kits}

Bioanalyzer DNA High sensitivity kit Immobilon Western HRP Substrate innuPREP RNA Mini Kit 2.0

KAPA Hyper Prep kit

NEXTflex ${ }^{\mathrm{TM}}$ Rapid Illumina Directional

RNA-Seq Library Prep Kit

Qubit dsDNA HS assay

Bioanalyzer DNA High sensitivity kit SuperSignal ${ }^{\mathrm{TM}}$ West Femto Maximum

Sensitivity Substrate
Agilent Technologies, Santa Clara, USA

Millipore, Billerica, USA

Analytik Jena AG, Jena, Germany

Roche, Pleasanton, USA

Bio Scientific Corporation, Austin, USA Invitrogen $\mathrm{GmbH}$, Karlsruhe, Germany Agilent Technologies, Santa Clara, USA

Thermo Fisher Scientific, Waltham, USA

\subsection{Nucleic acids}

\subsubsection{RT-PCR primers}

\begin{tabular}{|l|l|l|}
\hline \multirow{2}{*}{ Gene } & Primer sequence (5'-3') & Reference \\
\hline \multirow{2}{*}{ Actin_ChIP } & GAGTCTACACGCTAGGCGTAA & F. Wegwitz \\
\cline { 2 - 3 } & CTCGTGGCTAGTACCTCACTG & F. Wegwitz \\
\hline \multirow{2}{*}{ E-cadherin } & CCTGGCACTGGTATCTCTTCA & E. Lenfert \\
\cline { 2 - 3 } & AGCCATTGCCAAGTACATCCTC & E. Lenfert \\
\hline \multirow{2}{*}{ Epcam } & GAGTCCGAAGAACCGACAAGG & G. Tolstonog \\
\cline { 2 - 3 } & CTGATGGTCGTAGGGGCTTTC & G. Tolstonog \\
\hline Evx1_ChIP & TGGCAGCAGCCTTAAACCTT & This study \\
\hline
\end{tabular}




\begin{tabular}{|c|c|c|}
\hline & AGCTGCAGTAGACCGTTGAC & This study \\
\hline \multirow{2}{*}{ Ezh2 } & TCCATGCAACACCCAACACA & W. Xie \\
\hline & АACTCСТTAGCTCССТССАGAT & W. Xie \\
\hline \multirow{2}{*}{ Hdac4 } & CTGTGGAGCTGCTGAATCCT & This study \\
\hline & TTCCAAGGGCAGTGAGAACT & This study \\
\hline \multirow{2}{*}{ Hdac7 } & TCTCTTCCTGGCAGGCTTAC & This study \\
\hline & AGTTGCCGAAGTTCTTGCTC & This study \\
\hline \multirow{2}{*}{ Hdac8 } & ATGACTGTGTCCCTGCACAA & This study \\
\hline & CTGAATGGGCACATTGACAC & This study \\
\hline \multirow{2}{*}{ N-cadherin } & GCGCAGTCTTACCGAAGGATG & This study \\
\hline & ATACACCGTGCCGTCCTCGT & This study \\
\hline \multirow{2}{*}{ Nfatc1 } & GCCTTTTGCGAGCAGTATCT & E. Hessmann \\
\hline & GCTGCCTTCCGTCTCATAGT & E. Hessmann \\
\hline \multirow{2}{*}{ Nfatc2 } & GAACAACATGAGAGCCACCA & This study \\
\hline & GTGTTCTTCCTGCCGATGTC & This study \\
\hline \multirow{2}{*}{ Rplpo } & GATTCGGGATATGCTGTTGG & This study \\
\hline & GCCTGGAAGAAGGAGGTCTT & This study \\
\hline \multirow{2}{*}{ Snai1 } & CTGGTGAGAAGCCATTCTCCT & E. Lenfert \\
\hline & CCTGGCACTGGTATCTCTTCA & E. Lenfert \\
\hline \multirow{2}{*}{ Suz12 } & AGCATCAAAAGCTTGTCTGCAC & W. Xie \\
\hline & ACTTTCACAAGCAGGACTTCCA & W. Xie \\
\hline \multirow{2}{*}{ Twist2 } & GGCCGCCAGGTACATAGAC & G.Tolstonog \\
\hline & GTAGCTGAGACGCTCGTGA & G.Tolstonog \\
\hline \multirow{2}{*}{ Vimentin } & CGGCTGCGAGAGAAATTGC & G.Tolstonog \\
\hline & CCACTTTCCGTTCAAGGTCAAG & G.Tolstonog \\
\hline \multirow{2}{*}{ Zeb1 } & CACCAGAAGCCAGCAGTCAT & This study \\
\hline & CGTTCTTCTCATGGCGGTACT & This study \\
\hline
\end{tabular}

\subsection{2 siRNAs for transient gene silencing}

\begin{tabular}{|l|l|l|l|}
\hline Gene & Sequence (5'-3') & Cat. no. & Supplier \\
\hline Ezh2 & $\begin{array}{l}\text { GGAAAGAACUGAAACCUUA } \\
\text { CAGAAGAGCUGAUGAAGUA } \\
\text { AGAAAGAUCUAGAGGAUAA } \\
\text { GGAGGGAGCUAAGGAGUUU }\end{array}$ & M-040882-00 & Dharmacon \\
\hline EZH2 & $\begin{array}{l}\text { GAGGACGGCUUCCCAAUAA } \\
\text { GCUGAAGCCUCAAUGUUUA } \\
\text { UAACGGUGAUCACAGGAUA } \\
\text { GCAAAUUCUCGGUGUCAAA }\end{array}$ & L-00421800 & Dharmacon \\
\hline Hdac4 & $\begin{array}{l}\text { GCUCAAGGCUUAAGCAGAA } \\
\text { CCAAGAAACUUACCCGUAU } \\
\text { GCAGAGGAUCCACCAGUUA } \\
\text { GUGGAUAGCGACACCAUAU }\end{array}$ & M-043626-01 & Dharmacon \\
\hline Hdac7 & CCGAAAGGCUUCCCUAGAG & M-040703-01 & Dharmacon \\
\hline
\end{tabular}




\begin{tabular}{|c|c|c|c|}
\hline & $\begin{array}{l}\text { UGACGCAGCAGUUGAUGAA } \\
\text { GCUACAGCAACACGGCAAA } \\
\text { GAGUGGGACCUAUGGCGAA }\end{array}$ & & \\
\hline Hdac8 & $\begin{array}{l}\text { CAUCGAAGGUUAUGACUGU } \\
\text { GACGGGAAGUGUAAAGUAG } \\
\text { CUACGUGGAUUUGGAUCUA } \\
\text { CUGAUUAUGUGCUGGAAAU }\end{array}$ & M-058613-01 & Dharmacon \\
\hline Nfatc1 & $\begin{array}{l}\text { GCCAUAACUUUCUGCAAGA } \\
\text { GGGCAAGCAUCACGGAGGA } \\
\text { CCAACUACUCCUACCCAUA } \\
\text { ACGGUUACUUGGAGAAUGA }\end{array}$ & M-054700-01 & Dharmacon \\
\hline NFATc1 & & 40657 & Thermo Fisher \\
\hline NT5 & & D-001206-13 & Dharmacon \\
\hline Suz12 & $\begin{array}{l}\text { GGACCUACAUUACAAUUUA } \\
\text { GAUGUAAGUUGUCCAAUAA } \\
\text { GCAGGUUCAUCUUCAAUUA } \\
\text { GCACAGAACUCUUACUUAC }\end{array}$ & M-040180-00 & Dharmacon \\
\hline
\end{tabular}

\subsection{Proteins}

\subsubsection{Enzymes}

Proteinase- $\mathrm{K}$

Reverse Transcriptase (M-MuLV)

RNase A

RNase Inhibitor

Taq DNA Polymerase
Life Technology, Carlsbad, USA

New England Biolabs, FFM

Qiagen $\mathrm{GmbH}$, Hilden

New England Biolabs, FFM

Prime Tech, Minsk, Belarus

\subsubsection{Antibodies}

Antibodies were diluted as mentioned below in 5\% milk or BSA. Primary antibodies for Western Blot contained $0.01 \%$ sodium azide.

Primary antibodies

\begin{tabular}{|l|l|l|l|l|l|l|l|}
\hline Antibody & $\begin{array}{l}\text { Cat. } \\
\text { no./Clone. } \\
\text { no. }\end{array}$ & Source & $\begin{array}{l}\text { Western } \\
\text { blot }\end{array}$ & IHC & IF & ChIP & FACS \\
\hline
\end{tabular}




\begin{tabular}{|l|l|l|l|l|l|l|l|}
\hline Actin & 8224 & Abcam & $1: 2000$ & & & & \\
\hline E-cadherin & $24 \mathrm{E} 10$ & $\begin{array}{l}\text { Cell } \\
\text { Signaling }\end{array}$ & $1: 1000$ & & & & \\
\hline Ezh2 & $5246 /$ D2C9 & $\begin{array}{l}\text { Cell } \\
\text { Signalling }\end{array}$ & $1: 1000$ & $1: 150$ & $1: 100$ & & \\
\hline $\begin{array}{l}\text { FITC-a- } \\
\text { EpCAM }\end{array}$ & 118207 & Biolegend & & & & & $1: 400$ \\
\hline H3 & 601902 & Biolegend & $1: 500$ & & & & \\
\hline H3K27ac & C15410196 & Diagenode & & & & $2 \mu \mathrm{g}$ & $2 \mu \mathrm{gg}$ \\
\hline H3K27me3 & C15410195 & Diagenode & & & & $2 \mu \mathrm{g}$ & $2 \mu \mathrm{g}$ \\
\hline N-cadherin & D4R1H & & $1: 1000$ & & & & \\
\hline Nfatc1 & 649601 & Biolegend & $1: 500$ & $1: 25$ & & & \\
\hline Suz12 & D39F6/ 3737 & $\begin{array}{l}\text { Cell } \\
\text { Signalling }\end{array}$ & $1: 1000$ & & $1: 100$ & & \\
\hline Vimentin & 18814 & $\begin{array}{l}\text { Cell } \\
\text { Signaling }\end{array}$ & $1: 1000$ & & & & \\
\hline
\end{tabular}

Secondary antibodies

\begin{tabular}{|l|l|l|l|l|}
\hline Antibody & $\begin{array}{l}\text { Cat. } \\
\text { no. }\end{array}$ & Source & $\begin{array}{l}\text { Western } \\
\text { blot }\end{array}$ & IF \\
\hline goat anti-mouse (IgG)HRP & $\begin{array}{l}\text { Sc- } \\
2004\end{array}$ & Santa Cruz & $1: 10000$ & \\
\hline goat anti-rabbit (IgG)HRP & $\begin{array}{l}\text { Sc- } \\
2005\end{array}$ & Santa Cruz & $1: 10000$ & \\
\hline donkey anti-goat (IgG)HRP & $\begin{array}{l}\text { Sc- } \\
2020\end{array}$ & Santa Cruz & $1: 10000$ & \\
\hline $\begin{array}{l}\text { Alexa Fluor } \\
\text { Rabbit IgG }\end{array}$ & A11008 Goat Anti- & Life Technologies & & $1: 10000$ \\
\hline $\begin{array}{l}\text { Alexa Fluor } \\
\text { Mouse IgG }\end{array}$ & A31570 Donkey Anti- & Life Technologies & & $1: 10000$ \\
\hline
\end{tabular}

\subsection{Cell culture}

\subsubsection{Cell lines}

\begin{tabular}{|l|l|l|}
\hline \multicolumn{1}{|c|}{ Cell line } & \multicolumn{1}{c|}{ Medium } & \multicolumn{1}{c|}{ Source } \\
\hline pG-2 & DMEM, GlutaMAX & F. Wegwitz (AG Wegwitz), Göttingen \\
\hline rG-2 & DMEM, GlutaMAX & G. Schmidt (AG Wegwitz), Göttingen \\
\hline MDA-MB-468 & RPMI-1640 & ATCC ${ }^{\circledR}$ HTB-132 \\
\hline
\end{tabular}




\begin{tabular}{|c|c|c|}
\hline MDA-MB-231 & RPMI-1640 & ATCC® HTB-26 \\
\hline HCC1806 & RPMI-1640 & ATCC $®$ CRL-2335 \\
\hline HCC70 & RPMI-1640 & ATCC $₫$ CRL-2315 \\
\hline HCC1937 & RPMI-1640 & ATCC $®$ CRL-2336 \\
\hline
\end{tabular}

\subsubsection{Media and reagents}

Each medium for cell culture contained 10\% FBS, $100 \mathrm{U} / \mathrm{ml}$ penicillin, $100 \mu \mathrm{g} / \mathrm{ml}$ streptomycin.

\begin{tabular}{|l|l|}
\hline \multicolumn{1}{|c|}{ Medium } & \multicolumn{1}{c|}{ Supplier } \\
\hline $\begin{array}{l}\text { Dulbecco's Modified Eagle Medium } \\
\text { (DMEM), high glucose, GutaMAX }{ }^{\text {TM }}\end{array}$ & Gibco $^{\circledR}$, Invitrogen GmbH, Karlsruhe \\
\hline RPMI-1640 & Gibco®, Invitrogen GmbH, Karlsruhe \\
\hline Fetal Bovine Serum (FBS) & $\begin{array}{l}\text { Thermo Scientific HyClone, Logan, } \\
\text { USA }\end{array}$ \\
\hline Penicillin/streptomycin (P/S) & Sigma-Aldrich Co., St. Louis, USA \\
\hline $0.05 \%$ Trypsin-EDTA & Gibco®, Invitrogen GmbH, Karlsruhe \\
\hline Opti-MEM & Life Technology, Carlsbad, USA \\
\hline
\end{tabular}

\subsection{Buffers}

\begin{tabular}{|l|l|l|}
\hline \multicolumn{1}{|c|}{ Buffer } & \multicolumn{2}{|c|}{ Ingredient } \\
\hline qPCR buffer & Tris/HCl, $\mathrm{pH} 8.8$ & $75 \mathrm{mM}$ \\
\cline { 2 - 3 } & $\left(\mathrm{NH}_{4}\right)_{2} \mathrm{SO}_{4}$ & $20 \mathrm{mM}$ \\
\cline { 2 - 3 } & $\mathrm{Tween}_{20}$ & $0.01 \%$ \\
\cline { 2 - 3 } & $\mathrm{MgCl}_{2}$ & $3 \mathrm{mM}$ \\
\cline { 2 - 3 } & dNTPs & $0.2 \mathrm{mM}$ \\
\cline { 2 - 3 } & Triton X-100 & $0.25 \%$ \\
\cline { 2 - 3 } & Taq polymerase & $20 \mathrm{U} / \mathrm{ml}$ \\
\cline { 2 - 3 } & SYBR Green I & $1: 80000$ \\
\cline { 2 - 3 } & Trehalose & $300 \mathrm{mM}$ \\
\hline
\end{tabular}




\begin{tabular}{|c|c|c|}
\hline \multirow[t]{2}{*}{ Blocking solution } & Milk powder & $5 \%(w / v)$ \\
\hline & in TBS-T 1x & \\
\hline \multirow[t]{4}{*}{ CAF stock solution (1) } & 5-Fluorouracil & $77 \mu \mathrm{M}$ \\
\hline & Doxorubicin & $0.92 \mu \mathrm{M}$ \\
\hline & Cyclophosphamide & $38 \mu \mathrm{M}$ \\
\hline & in cell culture medium & \\
\hline \multirow[t]{5}{*}{ ChIP wash buffer } & SDS & $0.01 \%(w / v)$ \\
\hline & Triton X-100 & $1.1 \%(\mathrm{v} / \mathrm{v})$ \\
\hline & EDTA & $1.2 \mathrm{mM}$ \\
\hline & Tris-HCl, $\mathrm{pH} 8.1$ & $16.7 \mathrm{mM}$ \\
\hline & $\mathrm{NaCl}$ & $167 \mathrm{mM}$ \\
\hline \multirow[t]{2}{*}{ Citric acid buffer, pH 6.0} & Citric acid & $12 \mathrm{mM}$ \\
\hline & Tri-sodium citrate & $100 \mathrm{mM}$ \\
\hline Cross-linking buffer & Formaldehyde in PBS & $37 \%$ \\
\hline \multirow[t]{2}{*}{ Crystal violet solution } & Crystal violet & $0.1 \%(w / v)$ \\
\hline & $\mathrm{EtOH}$ & $20 \%$ \\
\hline \multirow[t]{3}{*}{ DNA loading dye (6x) } & Sucrose & $40 \%(w / v)$ \\
\hline & Glycerol & $10 \%(\mathrm{v} / \mathrm{v})$ \\
\hline & Bromophenol blue & $0.25 \%(w / v)$ \\
\hline \multirow[t]{2}{*}{ FACS resuspension buffer } & FBS & $2 \%(v / v)$ \\
\hline & EDTA in PBS & $1 \mathrm{mM}$ \\
\hline \multirow[t]{7}{*}{ IP buffer } & $\mathrm{NaCl}$ & $5 \mathrm{M}$ \\
\hline & EDTA, pH 8.0 & $0.5 \mathrm{M}$ \\
\hline & Tris-HCl, $\mathrm{pH} 8.0$ & $1 \mathrm{M}$ \\
\hline & NP-40 & $10 \%(\mathrm{v} / \mathrm{v})$ \\
\hline & Sodium deoxycholate & $10 \%(w / v)$ \\
\hline & $\mathrm{NaF}$ & $0.5 \mathrm{M}$ \\
\hline & SDS & $10 \%(w / v)$ \\
\hline \multirow[t]{3}{*}{ Lämmli buffer (6x) } & DTT & $9.3 \%(w / v)$ \\
\hline & Tris, $\mathrm{pH} 6.8$ & $0.35 \mathrm{M}$ \\
\hline & Glycerol & $30 \%(v / v)$ \\
\hline
\end{tabular}




\begin{tabular}{|c|c|c|}
\hline & SDS & $10 \%(w / v)$ \\
\hline & Bromophenol blue & $0.02 \%(w / v)$ \\
\hline Nuclear preparation buffer & $\mathrm{NaCl}$ & $5 \mathrm{M}$ \\
\hline & EDTA (pH 8.0) & $0.5 \mathrm{M}$ \\
\hline & Tris- $\mathrm{HCl}(\mathrm{pH} 7.5)$ & $1 \mathrm{M}$ \\
\hline & NP-40 & $10 \%(\mathrm{v} / \mathrm{v})$ \\
\hline & Triton-X-100 (v/v) & $10 \%(\mathrm{v} / \mathrm{v})$ \\
\hline & $\mathrm{NaF}$ & $0.5 \mathrm{M}$ \\
\hline PBS for cell culture & PBS tablet & $1 x$ \\
\hline & $\mathrm{ddH}_{2} \mathrm{O}$ & $500 \mathrm{ml}$ \\
\hline PBS, pH 7.4 & $\mathrm{NaCl}$ & $137 \mathrm{mM}$ \\
\hline & $\mathrm{Na}_{2} \mathrm{HPO}_{4} .2 \mathrm{H}_{2} \mathrm{O}$ & $4.29 \mathrm{mM}$ \\
\hline & $\mathrm{KCl}$ & $2.68 \mathrm{mM}$ \\
\hline & $\mathrm{KH}_{2} \mathrm{PO}_{4}$ & $1.47 \mathrm{mM}$ \\
\hline PBS-T & Tween-20 in PBS & $0.1 \%(w / v)$ \\
\hline RIPA buffer & NP-40 & $1 \%$ \\
\hline & SDS & $0.1 \%$ \\
\hline & Sodium deoxycholate in PBS & $0.5 \%$ \\
\hline RNA loading dye & Bromophenol blue & $0.1 \%(w / v)$ \\
\hline & DEPC water & $49.9 \%$ \\
\hline & Glycerol & $50 \%(w / v)$ \\
\hline Running buffer & Glycine & $200 \mathrm{mM}$ \\
\hline & Tris & $25 \mathrm{mM}$ \\
\hline & SDS (w/v) & $0.1 \%$ \\
\hline SDS-PAGE running buffer & Tris & $25 \mathrm{mM}$ \\
\hline & Glycerine & $86 \mathrm{mM}$ \\
\hline & SDS & $3.5 \mathrm{mM}$ \\
\hline SDS-PAGE separating gel & Acrylamide & $33 \%(v / v)$ \\
\hline & APS & $0.1 \%(\mathrm{w} / \mathrm{v})$ \\
\hline & SDS & $0.1 \%(w / v)$ \\
\hline & Tris/HCl, $\mathrm{pH} 8.8$ & $375 \mathrm{mM}$ \\
\hline
\end{tabular}




\begin{tabular}{|c|c|c|}
\hline & TEMED & $0.04 \%(v / v)$ \\
\hline \multirow[t]{5}{*}{ SDS stacking gel } & Acrylamide & $33 \%(v / v)$ \\
\hline & APS & $0.1 \%(w / v)$ \\
\hline & SDS & $0.1 \%(w / v)$ \\
\hline & Tris/HCl, ph 6.8 & $125 \mathrm{mM}$ \\
\hline & TEMED & $0.01 \%(\mathrm{v} / \mathrm{v})$ \\
\hline \multirow[t]{3}{*}{ TAE (50x) buffer } & Tris & $2 \mathrm{M}$ \\
\hline & Acetic acid & $1 \mathrm{M}$ \\
\hline & EDTA & $0.1 \mathrm{M}$ \\
\hline \multirow[t]{3}{*}{ TBE buffer } & Tris & $45 \mathrm{mM}$ \\
\hline & $\mathrm{Na}_{2} \mathrm{EDTA}$ & $1 \mathrm{mM}$ \\
\hline & Boric acid & $45 \mathrm{mM}$ \\
\hline \multirow[t]{4}{*}{ TBS, pH 7.4} & $\mathrm{NaCl}$ & $150 \mathrm{mM}$ \\
\hline & $\mathrm{KCl}$ & $2.68 \mathrm{mM}$ \\
\hline & $\mathrm{Na}_{2} \mathrm{HPO}_{4} \times 2 \mathrm{H}_{2} \mathrm{O}$ & $4.29 \mathrm{mM}$ \\
\hline & $\mathrm{KH}_{2} \mathrm{PO}_{4}$ & $1.47 \mathrm{mM}$ \\
\hline TBS-T & Tween-20 in TBS & $0.1 \%(w / v$ \\
\hline \multirow[t]{2}{*}{ TE buffer } & EDTA, pH 8.0 & $0.5 \mathrm{M}$ \\
\hline & Tris-HCl, pH 8.0 & $1 \mathrm{M}$ \\
\hline \multirow[t]{2}{*}{ Transfer buffer } & 10x western salts & $10 \%(\mathrm{v} / \mathrm{v})$ \\
\hline & Methanol & $20 \%(v / v)$ \\
\hline \multirow[t]{3}{*}{ Western salts (10x), pH 8.3} & Glycine & $1.92 \mathrm{M}$ \\
\hline & SDS & $0.02 \%(w / v)$ \\
\hline & Tris $/ \mathrm{HCl}$ & $250 \mathrm{mM}$ \\
\hline
\end{tabular}

\subsection{Softwares and online tools}

Bio-Rad CFX Manager

DESeq2 package
Bio-Rad Laboratories, Hercules, USA

https://bioconductor.org/packages/release

/bioc/html/DESeq2.html 


$\begin{array}{ll}\text { DiffBind package } & \begin{array}{l}\text { http://bioconductor.org/packages/release/bioc/ } \\ \text { html/DiffBind.html }\end{array} \\ \text { Enrichr } & \text { https://amp.pharm.mssm.edu/Enrichr/ } \\ \text { Galaxy Deeptools } & \text { http://deeptools.ie-freiburg.mpg.de/ } \\ \text { Gene Set Enrichment Analysis } & \text { http://software.broadinstitute.org/gsea/index.jsp } \\ \text { GIMP 2.10.6 } & \text { https://www.gimp.org/ } \\ \text { GraphPad Prism } & \text { https://www.graphpad.com/scientific- } \\ & \text { software/prism/ } \\ \text { GREAT analysis software } & \text { http://bejerano.stanford.edu/great/public/html/ } \\ \text { Image Lab Version 5.2 } & \text { Bio-Rad Laboratories, Hercules, USA } \\ \text { Kaplan-Meier plotter } & \text { http://kmplot.com/analysis/index.php?p } \\ & =\text { background } \\ \text { MERAV } & \text { http://merav.wi.mit.edu/ } \\ \text { NCBI primer-BLAST } & \text { www.ncbi.nlm.nih.gov/tools/primer- } \\ \text { blast/Primer/designing tool/ }\end{array}$

\section{Methods}

\subsection{Cell culture}

\subsubsection{Cell maintenance}

pG-2 and rG-2 cells were cultured in DMEM GlutaMAX, whereas MDA-MB-468, MDAMB-231, HCC1937, HCC1806, HCC70 were maintained in RPMI- 1640 at $37^{\circ} \mathrm{C}$ and $5 \%$ 
$\mathrm{CO}_{2}$. All media were supplemented with $10 \%$ FBS, $100 \mathrm{U} / \mathrm{ml}$ penicillin, $100 \mu \mathrm{g} / \mathrm{ml}$ streptomycin. For cell passaging $1 \times$ PBS and $0.05 \%$ Trypsin-EDTA were used.

\subsubsection{Reverse transfection}

Cells were reverse transfected with siRNA in 6-well plates using Lipofectamine ${ }^{\circledR}$ RNAiMAX, according to the manufacturer's instructions. $1.5 \mu \mathrm{l}$ of $20 \mu \mathrm{M}$ siRNA was mixed gently with $5 \mu$ Lipofectamine ${ }^{\circledR}$ RNAiMAX and $500 \mu$ l of Opti-MEM and incubated for 20 minutes at room temperature. After transferring $500 \mu \mathrm{l}$ of the prepared transfection mixture to the well, $1.5 \mathrm{ml}$ of Opti-MEM with 350,000 cells were added and the plate was kept in the incubator in normal cell culture conditions. After 24 hours, the cells were used for other assays.

\subsubsection{Cell proliferation assay}

24-well plates with seeded 10.000 cells per well were used to assess cell proliferation. If the cells were already transfected with siRNA, after 48 hours cells were treated with chemotherapy. In the case of inhibition, cells were treated with inhibitor the day after seeding and incubated for 48 hours, for the next 2 days inhibition and chemotherapy treatment was performed or inhibition alone. For the next 48 hours, cells were treated again with inhibitor and afterwards with fresh medium only. Every 2 days cells proliferation was measured using the Celigo ${ }^{\circledR}$ Adherent Cell Cytometer for a total of 1 2 weeks, depending on the treatment and the cell line. On the last day of the experiment, the cells were fixed with $100 \%$ methanol and stained using $0.1 \%$ crystal violet in $\mathrm{EtOH}$.

\subsubsection{Migration assay}

Scratch assay. 400.000 cells, previously transfected with siRNA or treated with inhibitor, were seeded on each well. The next day, if the cells were $95 \%$ confluent, scratches on monolayer of cells were performed using pipette tips. Immediately, medium was changed for fresh medium with no FBS. Photos were taken after 0 and 12 hours and analyzed via ImageJ. 
Boyden chamber assay. Boyden chamber inserts (8.0- $\mu$ l track-etched membrane cell culture inserts) were equilibrated with serum free medium supplemented with penicillin and streptomycin. Inserts were transferred into 24-well plates containing $500 \mu \mathrm{la}$ complete cell culture medium. 50.000 cells in $300 \mu \mathrm{l}$ serum free medium were seeded into the inserts. After 48 hours, the inserts were washed with PBS and cells on the upper site of the insert were carefully removed. Cells on the lower side of the insert were fixed using $4 \%$ PFA for 10 min and stained with $1 \%$ crystal violet in $20 \% \mathrm{EtOH}$ for 20 min. After drying, photos were taken and analyzed using ImageJ.

\subsubsection{Colony formation assay}

2.000 cells after siRNA transfection or subsequent treatments were seeded per well in a 6-well plate. The colonies were stained with crystal violet and counted manually at the end of the experiment.

\subsection{Molecular biology}

\subsubsection{RNA isolation}

From a 6-well plate, the cells were washed with PBS and resuspended in $500 \mu \mathrm{l}$ of TRIzol@. According to manufacturer's manual, $200 \mu \mathrm{l}$ chloroform was added and vortexed for 15 seconds. After centrifuging at $4^{\circ} \mathrm{C}, 12.000 \mathrm{~g}$ for $15 \mathrm{~min}$, the aqueous phase was taken and vigorously mixed with an equal volume of isopropanol. After minimum 1 hour of precipitation at $-80^{\circ} \mathrm{C}$, the samples were centrifuged at $4{ }^{\circ} \mathrm{C}, 12,000$ $\mathrm{g}$ for $15 \mathrm{~min}$. The RNA pellets were washed $2 \mathrm{x}$ with cold $70 \%$ ethanol in DEPC water. Finally, the supernatant was discarded completely and the RNA pellets were air dried for around 10 minutes and diluted in $40 \mu \mathrm{LEPC}$ water. The RNA quantification was performed using the Spectrophotometer, Denovox. Alternatively, the innuPREP RNA Mini Kit 2.0 from Analytik Jena AG was used for RNA isolation and the manufacturer's procedure was followed. 


\subsection{2. cDNA synthesis}

For the cDNA synthesis, a mixture of $0.5-1 \mu \mathrm{g}$ of RNA, $2 \mu \mathrm{l} 60 \mu \mathrm{M}$ random primers, 1 $\mu \mathrm{M}$ dNTPs and DEPC water in a total volume of $10 \mu \mathrm{l}$ was prepared and incubated at $65^{\circ} \mathrm{C}$ for $5 \mathrm{~min}$ and cooled down on ice. $2 \mu \mathrm{l} 10 \times \mathrm{M}$-MuLV buffer, $0.25 \mu \mathrm{l}$ [10 U] RNase inhibitor, $1 \mu \mathrm{l}$ M-MuLV reverse transcriptase and DEPC in a volume of $10 \mu \mathrm{l}$ were added to each reaction mix. The samples were incubated at $25^{\circ} \mathrm{C}$ for $5 \mathrm{~min}, 42^{\circ} \mathrm{C}$ for $1 \mathrm{~h}$ and $95^{\circ} \mathrm{C}$ for $5 \mathrm{~min}$. The $\mathrm{cDNA}$ samples were diluted to $5 \mathrm{ng} / \mu \mathrm{l}$ and stored at $-20^{\circ} \mathrm{C}$ or $-80^{\circ} \mathrm{C}$.

\subsubsection{Quantitative real-time PCR}

To quantify relative gene expression, $1 \mu \mathrm{l}$ of cDNA was used in master mix containing $14 \mu \mathrm{l} 2 \mathrm{x}$ qPCR mix, $9 \mu \mathrm{lddH} 2 \mathrm{O}$ and $1 \mu \mathrm{l}$ primers in one reaction. Firstly, the cDNA was denatured at $95^{\circ} \mathrm{C}$ for $2 \mathrm{~min}$, then 40 cycles of $95^{\circ} \mathrm{C}$ for $15 \mathrm{~s}$ and $60^{\circ} \mathrm{C}$ for $30 \mathrm{sec}$ were run. Finally, the melting curve analysis was generated with SYBR green by heating from $60^{\circ} \mathrm{C}$ to $95^{\circ} \mathrm{C}$ with one read every $0.5^{\circ} \mathrm{C}$. Based on the standard curve, the results were quantified.

\subsubsection{Crosslinking}

For ChIP, pG2 cells cultured with or without chemotherapy treatment for 48 hours on $15 \mathrm{~cm}$ plates were used. The cells were crosslinked with $1 \%$ formaldehyde in PBS for $20 \mathrm{~min}$, followed by quenching with $125 \mathrm{mM}$ glycine for $5 \mathrm{~min}$. After washing the cells $2 x$ with cold PBS, $1 \mathrm{ml}$ of nuclear preparation buffer containing a proteinase inhibitor cocktail was added. Scraped cells were centrifuged at $12.000 \mathrm{~g}$ for 2 min at $4{ }^{\circ} \mathrm{C}$ and the nuclear pellet was washed with nuclear preparation buffer.

\subsubsection{DNA content determination}

$50 \mu \mathrm{l}$ of the supernatant was transferred from the crosslinking step prior to the last centrifugation. After adding $250 \mu \mathrm{l}$ of sonication buffer-1 and $1 \mu \mathrm{l}$ of Proteinase K (20 $\mathrm{mg} / \mathrm{ml}$ ), the samples were incubated at $65^{\circ} \mathrm{C}$ overnight, $800 \mathrm{rpm} .250 \mu \mathrm{l}$ of distilled water, $25 \mu \mathrm{l}$ of $8 \mathrm{M} \mathrm{LiCl}$ and $2 \mu \mathrm{l}$ of colorless co-precipitant were added to each tube. 
Upon phenol/chloroform/isoamylic alcohol extraction, samples were vortexed and centrifuged $12,000 \mathrm{~g}, 2 \mathrm{~min}, 4^{\circ} \mathrm{C}$ and the aqueous phase was transferred to a fresh tube. To precipitate, $1 \mathrm{ml}$ of isopropanol was added to each sample, which was then incubated for $1 \mathrm{~h}$ at $-80^{\circ} \mathrm{C}$. After $30 \mathrm{~min}$ of centrifugation at $15.000 \mathrm{~g}, 4^{\circ} \mathrm{C}$, the pellet was washed $2 x$ with $70 \% \mathrm{EtOH}$. Afterwards, the final pellets were resuspended in $50 \mu \mathrm{l}$ Tris $10 \mathrm{mM} \mathrm{pH} 8$ with RNase A $100 \mu \mathrm{g} / \mathrm{ml}$ and quantified with the spectrophotometer.

\subsubsection{Sonication}

Based on the DNA concentration, the samples were diluted to $500 \mu \mathrm{g} / \mathrm{ml}$ and incubated at $4^{\circ} \mathrm{C}$ for $15 \mathrm{~min}$ on the wheel. The samples were sonicated using the Bioruptor Pico for $5,10,15,20,25$ and 30 cycles with $30 \mathrm{sec}$ on/off duty time. After centrifuging at $10.000 \mathrm{~g}, 10 \mathrm{~min}, 4^{\circ} \mathrm{C}$, a fraction of the supernatants was taken for a shearing check.

\subsubsection{Shearing check}

$100 \mu \mathrm{l}$ of sonication buffer- 1 and $1 \mu \mathrm{l}$ of proteinase $\mathrm{K}$ were added to each sample, which were then incubated at $65^{\circ} \mathrm{C}$ for $4 \mathrm{~h}, 800 \mathrm{rpm}$. Next, $100 \mu \mathrm{l}$ water, $10 \mu \mathrm{l} 8 \mathrm{M} \mathrm{LiCl}$ and $2 \mu \mathrm{l}$ PINK precipitant were added. Phenol/chloroform/isoamylic alcohol was added to each sample, followed by 30 s vortexing and centrifuging at $2 \mathrm{~min}, 15000 \mathrm{~g}$. To the aqueous phase, $1 \mathrm{ml} \mathrm{EtOH}$ was added and after $1 \mathrm{~h}$ of incubation at $-80^{\circ} \mathrm{C}$, the samples were centrifuged for $30 \mathrm{~min}, 15000 \mathrm{~g}, 4^{\circ} \mathrm{C}$. The final pellet was resuspended in $15 \mu \mathrm{l}$ Tris 10 $\mathrm{mM} \mathrm{pH} 8.0$ with RNase A $100 \mu \mathrm{g} / \mathrm{ml}\left(1 \mathrm{~h}, 37^{\circ} \mathrm{C}, 700 \mathrm{~g}\right)$. The samples were mixed with $3 \mu \mathrm{l}$ of loading dye and run on a 1.5\% agarose gel using $1 \times$ TAE buffer.

\subsubsection{Pre-clearing and chromatin immunoprecipitation}

For pre-clearing, $100 \mu \mathrm{l}$ of a $50 \%$ sepharose slurry were added and the samples were incubated for $1 \mathrm{~h}, 4^{\circ} \mathrm{C}$. After centrifuging at $12000 \mathrm{~g}, 4^{\circ} \mathrm{C}$, the supernatant was collected and immunoprecipitation and input samples were prepared. Aliquots of chromatin were filled to $500 \mu \mathrm{l}$ with IP buffer with protease inhibitors and appropriate antibodies (see: Materials) with overnight incubation. On the following day, $30 \mu$ of protein A-sepharose was added and the samples were incubated for $2 \mathrm{~h}$ at $4^{\circ} \mathrm{C}$ with rotation. The ChIP complexes washed with IP buffer, wash buffer and TE buffer. 


\subsubsection{DNA isolation}

Immunoprecipitated chromatin was treated with $50 \mu \mathrm{l}$ of $10 \mu \mathrm{g}$ RNase A diluted in Tris $10 \mathrm{mM} \mathrm{pH} 8$ for $30 \mathrm{~min}$, at $37^{\circ} \mathrm{C}$. $50 \mu \mathrm{l}$ of sonication buffer was added on the beads with $1 \mu \mathrm{l}$ proteinase $\mathrm{K}$ with overnight incubation at $65^{\circ} \mathrm{C}$ with shaking. After centrifuging ( $2.000 \mathrm{~g}, 2 \mathrm{~min}$, at room temperature), the supernatant was taken and $10 \mu \mathrm{l}$ Tris $10 \mathrm{mM}$ $\mathrm{pH} 8$ was added, mixed and centrifuged, $2 \min 15.000 \mathrm{~g}$. The aqueous phase was taken and to precipitate, $1 \mathrm{ml} \mathrm{EtOH}$ was used for $2 \mathrm{~h}$ at $-80^{\circ} \mathrm{C}$. Next the samples were centrifuged for $30 \mathrm{~min}, 15.000 \mathrm{~g}, 4^{\circ} \mathrm{C}$ and washed with $1 \mathrm{ml} 70 \% \mathrm{EtOH}$. The DNA was resuspended in $40 \mu \mathrm{l} \mathrm{H}_{2} 0.5 \mu \mathrm{l}$ of each sample was used for ChIP-qPCR.

\subsubsection{ChIP-seq library preparations}

ChIP-seq libraries were prepared using the KAPA Hyper Prep kit according to manufacturer's protocol. The libraries were purified using 1 X AMPure $®$ XP beads on a magnetic stand. The quality of the samples was determined using Bioanalyzer with assessment of the fragment length (around $300 \mathrm{bp}$ ). A $2 \mathrm{nM}$ pool of ChIP DNA libraries were sequenced by the Transcriptome and Genome Analysis Laboratory in Göttingen (HiSeq 4000).

\subsubsection{ChIP-seq analysis}

For ChIP-seq analysis, the Galaxy server was used. ChIP-seq reads of two biological replicates for each condition in the experiment were used. FASTQ quality check (FastQC) of raw data was followed by Mapping, BamCoverage and Peak Calling. The sequenced reads were aligned to the mouse reference genome (mm9) using Bowtie2 (http://bowtie-bio.sourceforge.net/bowtie2/index.shtml). Sequence Alignment Map (SAM) files were converted to Binary Alignment Map (BAM) files using SAMtools ( $H$. $\mathrm{Li}$ et al., 2009). After merging replicates (BAM files), Model-based Analysis of ChIP-seq 2 (MACS2) was used for peak calling to identify enriched regions (Zhang et al., 2008). The next step was to generate a coverage file of the reads using bamCoverage with $500 \mathrm{bp}$ bin size and reads per kilobase per million (RPKM) normalization. Through 
conversion of BAM files to bigwig files, we could visualize ChIP-seq data. Further analysis was performed using computeMatrix, plotHeatmap and plotProfile. Additionally, differential binding analysis was performed for H3K27ac and H3K27me3 binding sites using the $\mathrm{R}$ package DiffBind (Stark \& Brown, 2011). Enrichr was performed based on significantly enriched genomic regions.

\subsection{Protein analysis}

\subsubsection{Protein harvesting}

Cells were washed with PBS and scraped in ice-cold RIPA buffer with a protease inhibitors cocktail: $1 \mathrm{mM}$ Pefabloc, $1 \mathrm{ng} / \mathrm{\mu l}$ Aprotinin/Leupeptin, $10 \mathrm{mM} \mathrm{BGP,} 1 \mathrm{mM}$ NEM and $8 \mathrm{M}$ urea ( $1 / 3$ of the final volume). The samples were sonicated for 10 cycles at 30 sec on/off duty time using Biorupter Pico sonicator. After centrifugation for $10 \mathrm{~min}$, $12,000 \mathrm{~g}, 4^{\circ} \mathrm{C}$, the protein samples were mixed with Lämmli buffer (Laemmli, 1970) and cooked for $5 \mathrm{~min}$ at $95^{\circ} \mathrm{C}$. Protein samples with the same concentration, were separated using polyacrylamide gel during SDS-PAGE electrophoresis. Gels were run using running buffer at range of $70-120 \mathrm{~V}$.

\subsubsection{Western blot}

For an electrophoretic protein transfer (Towbin, 1979), nitrocellulose membranes were used. After the transfer, the membranes were blocked in 5\% milk in TBS-T for $1 \mathrm{~h}$ and incubated overnight in primary antibody at $4^{\circ} \mathrm{C}$. Following, the membranes were washed with TBS-T and incubated for $1 \mathrm{~h}$ in secondary antibody diluted. After washing the membranes 3 times washing in TBS-T for 5 minutes each, they were developed using HRP signal and the western blot imager Biorad.

\subsection{Stainings}

\subsubsection{Crystal violet staining}

To analyze cell proliferation and colony formation, the cells were stained with $0.1 \%$ or $1 \%$ crystal violet in $20 \% \mathrm{EtOH}$ (modified from (Saotome, 1989)). The cells were washed 
with PBS, fixed with $100 \%$ methanol for 5 min and stained with $0.1 \%$ crystal violet for $20 \mathrm{~min}$, room temperature. After drying, the plates were scanned and analyzed.

\subsubsection{IHC staining}

Tumor sections were deparaffinized after melting for $10 \mathrm{~min}$ at $48^{\circ} \mathrm{C}$. Following, they were incubated in xylene for $20 \mathrm{~min}$, xylene 1:1 with $100 \% \mathrm{EtOH}, 100 \%$ isopropanol, $\mathrm{EtOH} 100 \%, 90 \%, 70 \%$ each for 5 min. After washing, the sections were cooked with EDTA or citric acid containing buffer for $10 \mathrm{~min}$. Then, endogenous peroxidase was blocked with $3 \%$ superoxide in PBS for $45 \mathrm{~min}$. The samples were washed in PBS and blocked with $3-5 \%$ BSA diluted in PBS for $1 \mathrm{~h}$, room temperature. Primary antibodies diluted in PBS were applied on top of the sections, overnight at $4^{\circ} \mathrm{C}$. To proceed, washing and biotinylated secondary antibodies incubation was carried out. After $1 \mathrm{~h}$, sections were washed and treated with avidin 1:1000 in PBS with 90 min incubation and washing afterwards. Development was performed using DAV until strong signal appeared. The sections were counterstained with hematoxylin and eosin. Finally, the samples were incubated in an inverted alcohol series and mounted.

\subsubsection{Immunofluorescence staining}

Cells were grown on coverslips in 24-well plates and later washed with PBS and fixed using $4 \%$ paraformaldehyde for 10 minutes and washed again. $0.1 \%$ Triton X-100 was used for 10 min to permeabilize the cells followed by washing. For the blocking step, the cells were incubated in 10\% BSA for $30 \mathrm{~min}$ followed by primary antibody overnight, $4^{\circ} \mathrm{C}$. On the following day, the cells were washed and incubated with the corresponding conjugated secondary antibody for $1 \mathrm{~h}$ at room temperature. Washing was followed by incubation with DAPI in PBS for 5 min and mounting. Images were taken using the AXIO Scope.A1 microscope. 


\subsubsection{Flow cytometry}

The cells were treated with cyclosporine $\mathrm{A}$ or thapsigargin in different concentrations for $48 \mathrm{~h}$ on 6 -well plates. After trypsinization, the cells were resuspended in $1 \mathrm{ml}$ DMEM with $10 \% \mathrm{FCS}, 100 \mathrm{U} / \mathrm{ml}$ penicillin and $100 \mu \mathrm{g} / \mathrm{ml}$ streptomycin. 200,000 of filtered cells were collected by centrifugation at $350 \mathrm{~g}$ for $5 \mathrm{~min}$ and resuspended in $100 \mu \mathrm{l}$ of a staining solution. To monitor EpCAM expression, the cells were stained with $2.5 \mu \mathrm{l}$ FITC-anti EpCAM (Biolegend) conjugated antibody in 1 ml MACS buffer. After 20 min of incubation in the dark, the cells were centrifuged for $5 \mathrm{~min}, 350 \mathrm{~g}$. The samples were resuspended in $500 \mu \mathrm{l}$ MACS buffer and $200 \mu \mathrm{l}$ of each sample were loaded in a 96well plate. FITC intensity was measured using the Guava EasyCyte plus (Guava Technologies) flow cytometer.

\subsection{Statistical analysis}

To create graphs, GraphPad Prism version 4.03 has been used for in this study. Pvalues were determined using Student's t-test $\left({ }^{* * *} p<0.001,{ }^{* *} p<0.01,{ }^{*} p<0.05\right)$. 


\section{Results}

Materials and methods (Section number 2 and 3) were prepared together for Chapter I and Chapter II part (Section number 4) of the thesis. The manuscript presented below was prepared based on Clinical Epigenetics Journal guidelines. In Chapter I and Chapter II short discussions are included. The overall discussion (General discussion) of all thesis results was shown below Chapter II. The overall references for all Chapters are indicated at the end of this thesis (Section Bibliography).

\subsection{Chapter I Manuscript}

Reduction of PRC2/EZH2 activity can promote better survival of TNBC cancer cells in a context-specific manner

Iga K. Mieczkowska', Geske E. Schmidtt, ${ }^{1}$, Lukas C. Müller-Kirschbaum ${ }^{1}$, Garyfallia Pantelakos Prokaki ${ }^{1}$, Evangelos Prokakis ${ }^{1}$, Taras Velychko', Frauke Alves $^{3}$, Madhobi Sen ${ }^{1}$, Florian Wegwitz ${ }^{1}$

1. Clinic for General, Visceral and Pediatric Surgery, University Medical Center Göttingen

2. Clinic for Gastroenterology, University Medical Center Göttingen

3. Molecular Imaging in Oncology, Tandemgroup Max Planck Institute for Experimental Medicine and University Medical Center Göttingen

\section{Own contribution:}

Planning and conducting the experiments in this project including Fig. I 1B, E-G, Fig. I 2D, F, G, Fig. I 3, Fig. I 4, Fig. I 5A-C, Fig. 6 I A-F, Fig. I S1, Fig. I S2, Fig. I S3, Fig. I S5. Cell culture assays (proliferation, migration, colony formation), treatments, ChIPseq, western blot, RT-qPCR and stainings. Complete figure layout and writing the manuscript was performed under the supervision of Dr. Florian Wegwitz. 
BACKGROUND: Breast cancer is the most common cancer occurring in women. Recent advances in early diagnosis and development of targeted therapies greatly improved the survival rate of breast cancer patients. However, conventional cytotoxic chemotherapies remain often the only treatment option for patients suffering from cancer subtypes where targeted therapies are not viable. Furthermore, the development of resistance is frequent and commonly followed by fatal consequences. In this study, we investigated epigenetic mechanisms underlying tumor cells surviving a combinatory chemotherapy treatment as potential targets to increase cytotoxic efficiency.

METHODS: Murine basal-like WAP-T mammary carcinoma cells and human triplenegative cell lines were utilized to study processes involved in cancer cell survival to a cyclophosphamide/doxorubicin/5-fluorouracil treatment. We performed high throughput RNA- and ChIP-sequencing analyses to assess transcriptome wide gene expression changes and underlying epigenetic regulatory mechanisms in cells surviving chemotherapy. To confirm our findings, we then employed several in vitro functional assay and corroborated our results on murine tissues and publicly available patient databases.

RESULTS: Epithelial to mesenchymal transition (EMT) and increased stemness were tightly associated with survival of the cancer cells to chemotherapy. We identified a reduction of Polycomb Repressive Complex 2 (PRC2) activity via downregulation of the Ezh2, Suz12 and Rbbp7 expression in chemotherapy-treated WAP-T cells. Interestingly, siRNA and small molecule inhibition of PRC2 activity improved the proliferation and survival of murine and human cancer cells to cytotoxic treatment. Mechanistically, loss of PRC2 activity lead to the derepression of a set of genes through a switch from the repressive $\mathrm{H} 3 \mathrm{~K} 27$ me 3 to the activating H3K27ac mark at regulatory regions. We identified Nfatc1 as a gene upregulated by loss of PRC2 activity and directly implicated with the transcriptional changes happening upon survival the chemotherapy. Blocking NFATc1 activation reduced epithelial to mesenchymal transition and aggressiveness of TNBC cells. 
CONCLUSIONS: Our data demonstrates a previously unknown function of PRC2 maintaining low Nfatc1 expression levels and thereby repressing invasiveness of TNBC.

KEYWORDS: PRC2, EZH2, TNBC, chemotherapy, H3K27ac, H3K27me3, NFATc1

\section{Background}

Breast cancer (also termed mammary carcinoma) is the most common cancerous disease in women with over 2 million new cases in 2018 worldwide (World Health Organisation, 2018). The mortality of breast cancer patients has significantly decreased over the past decades, mostly because of early diagnosis improvements and the development of several targeted therapies. However, despite intensive efforts to combat the disease, breast cancer remains the first cancer-related cause of death among women. The prognosis of cancer patients is largely determined by the metastatic lesions and recurrent tumor growth. Today, approximately $25 \%$ of breast cancer patients still develop distant metastases and ultimately die of their disease (Mathiesen et al., 2012). Even when detected early and treated by standard breastconserving surgery, breast cancer has a recurrence rate of $5-10 \%$ within 10 years (Colzani et al., 2014; Fisher et al., 2002; Harbeck et al., 2019; Veronesi et al., 2002). The high incidence of breast cancer and the high mortality rate of the disease in relapsed patients necessitates the urgent development of improved treatment options.

Because of its highly heterogeneous nature, breast cancer is commonly classified into distinct disease subtypes with specific therapeutic approaches and outcome, based on expression of the receptor molecules ER (estrogen receptor) and PR (progesterone receptor) and HER-2 (human epidermal growth factor receptor 2) (Prat, Pineda, et al., 2015). Despite their great usefulness in the clinic, these histological parameters do not fully reflect the complexity of the disease. Progress in gene expression profiling lead to the definition of at least four different molecular subtypes of breast cancer with very different incidence, prognosis and response to treatments: Luminal A, Luminal B, HER-2 enriched and triple negative breast cancer 
(TNBC) (Perou et al., 2000; Prat, Fan, et al., 2015). The possibility to specifically inhibit the activity of ER, PR and/or HER-2 via targeted therapies greatly improved the therapeutic options and prognosis of mammary carcinomas subtypes expressing those receptors. Unfortunately, because of the lack of ER/PR and HER-2 expression, the group of triple negative breast cancers (TNBC), that accounts for approximatively $15 \%$ of all mammary carcinomas do not profit from these therapeutic advances. Mammary carcinomas are clinically treated with a combination of surgery, radiation, chemotherapy and/or targeted therapies (if available) depending on the type and stage of the disease. Here, a combination of cyclophosphamide, anthracycline (doxorubicin) and/or 5-fluorouracil (5-FU) (CAF) have been shown to display an increase in pathological response rates in TNBCs compared to the other subtypes (Carey et al., 2007; O. Gluz et al., 2009). Despite a good first response to cytotoxic therapies, a large fraction of TNBC rapidly develop resistance. Consequently, TNBCs show the highest rate of recurrence after treatment and the poorest prognosis among breast cancer diseases (Prat et al. 2015).

Mechanisms allowing a tumor cell to escape conventional chemotherapeutic treatments require fast adaption to hostile conditions. Acquisition of epithelialmesenchymal plasticity (EMP) and stemness have been identified as potential mechanisms responsible for cancer progression, development of chemotherapy resistance and increased metastatic features (Lu and Kang 2019; Ye and Weinberg 2015). Such alterable properties necessitate rapid reorganization of whole gene expression profiles. Because of the dynamic and reversible nature of epigenetic modifications, epigenetic processes represent very likely mechanisms controlling cellular plasticity. Thus, epigenetic players are attractive targets for the development of the new anti-cancer drugs (Mohammad et al. 2019; Wouters and Delwel 2016). Numerous publications reported the central role of epigenetic factors mediating the function of transcription factors during epithelial to mesenchymal transition (EMT) (Lu and Kang 2019; Wainwright and Scaffidi 2017). In a similar manner, epigenetic mechanisms were shown to be indispensable for the acquisition and maintenance of cancer stem cell (CSC) properties (Skrypek et al., 2017; Wainwright \& Scaffidi, 2017). The Polycomb repressive complexes 1 and 2 (PRC1 and PRC2, respectively) are two 
well characterized epigenetic factors repressing the expression of specific genes via histone post-translational modification. The canonical PRC2 core complex is composed of four subunits EZH1/EZH2, EED, SUZ12 and RBBP7. Through its catalytic subunit $\mathrm{EZH} 2$, the PRC2 catalyzes the di- and trimethylation of histone 3 at lysine 27 (H3K27me2 and H3K27me3, respectively) promoting thereby a compaction of the chromatin, and as a consequence, leading to the silencing of genes located in the given region (Antonysamy et al., 2013; Simon \& Kingston, 2013). Interestingly, PRC2 was shown to play an essential role in normal embryonal and adult stem cells homeostasis by maintaining self-renewal and pluripotency through repression of differentiation programs (Raphaël Margueron \& Reinberg, 2011; Vizán et al., 2015). In line with these observations, higher EZH2 expression levels were associated with increased cancer stem cell properties and poor prognosis in numerous cancer entities including malignancies of the breast (Wen, Cai, Hou, Huang, \& Wang, 2017). Furthermore, the enzymatic activity of the PRC2 complex was shown to actively promote EMT by positively regulating the expression of and cooperating with central EMT-transcription factors (EMT-TFs) like SNAl1 or ZEB1 (Herranz et al., 2008; Martínez-Fernández et al., 2015).

In the past, we developed and characterized the WAP-T mammary carcinoma mouse model to study the biology, progression and metastatic processes of TNBC (Lenfert et al., 2015; Maenz et al., 2015; Otto, Gruner, et al., 2013; Otto, Streichert, et al., 2013; Schulze-Garg et al., 2000; Wegwitz et al., 2010). In a former effort to understand the effects of a CAF therapy on WAP-T mammary carcinomas, we observed that the cytotoxic combination therapy was not able to eradicate the disease in vivo. Interestingly, surviving tumor cells displayed a more aggressive mesenchymallike phenotype with increased stem cell traits and showed a pronounced tendency to dissemination (Jannasch et al., 2015). Because of its good mimicking of the clinical situation, we utilized this model in the present study to get insight into the molecular mechanisms underlying acquisition of EMP and stemness upon chemotherapy treatment and allowing tumor cell survival. We identified here a previously unknown PRC2 function repressing EMT and cancer stem cell program in TNBC cells along a PRC2/NFATc1 axis. 


\section{Results}

WAP-T cells surviving CAF treatment gain stem cell and EMT properties in vitro.

The parental G-2 cell line (pG-2), established from a WAP-T mammary carcinoma (Wegwitz et al., 2010) was utilized to investigate the effects of a CAF combination therapy on TNBC in vivo and it was observed that tumor cells surviving the therapy gained stemness and mesenchymal-like characteristics (Jannasch et al., 2015). To get insights into the molecular pathways underlying the survival and the emergence of resistance to the CAF chemotherapy in vitro, we optimized in a first step the chemotherapy treatment settings of G-2 cells in the cell culture. Aim here was the identification of treatment conditions eradicating most of the tumor cells but allowing the survival and regrowth of a small tumor cell fraction, mimicking thereby the in vivo relapse situation. A combination therapy consisting of $312.5 \mathrm{ng} / \mathrm{ml}$ cyclophosphamide, $15,6 \mathrm{ng} / \mathrm{ml}$ doxorubicin and $312,5 \mathrm{ng} / \mathrm{ml} 5-\mathrm{FU}$, corresponding to the $1 / 32$ dilution of the therapy previously utilized in Jannasch et al in vivo, was identified as the best appropriate setting (Fig. I 1A). This treatment was adopted for the rest of the experiments in the present study and will be designated as CAF therapy. Interestingly, pG-2 cells surviving CAF-treatment acquired a more elongated morphology, characteristic for cells undergoing EMT (Fig. I 1B). A chemotherapy resistant variant of the pG-2 cells called rG-2 cells was established through several cycles of CAF treatments (see method section for more details). Strikingly, rG-2 cells harbor in basal growth condition a mesenchymal-like phenotype, supporting the potential implication of EMT mechanisms in resistance to CAF therapy (Fig. I S1A). We performed RNAsequencing (RNA-seq) and compared the transcriptome of pG-2 cells treated 48 hours with CAF to vehicle treated control cells (ctr). DeSeq2 analyses identified 1021 downregulated and 1448 upregulated genes (|Log2(Fold Change) $\mid>1$, padj<0.05) in CAF-treated cells (Fig. I 1C). To get insights into transcriptional program changes occurring during survival to the treatment, we performed Gene Set Enrichment Analyses (GSEA). Strikingly, we observed a strong enrichment of gene sets related to EMT, cancer aggressiveness and stemness (Fig. I 1D). Indeed, the well-known EMT markers Vimentin (Vim) and N-cadherin (Cdh2) and EMT-TFs Snai1, Twist2 and Zeb1 
were upregulated in surviving cells whereas the expression of both epithelial marker Ecadherin (Cdh1) and Epcam were strongly reduced (Fig. I 1E). The regulation of Vim, Twist2, Snai1, Zeb1 and Cdh1 was validated using qPCR (Fig. I 1G). In a similar manner, the expression of stem cell specific transcription factors was also found to be increased in CAF-treated cells (Fig. I 1F). Interestingly, rG-2 cells showed increased expression of several EMT and stem cell markers under basal culture conditions (Fig. I S1B). These results support previous in vivo studies (Jannasch et al., 2015) and further emphasize the implication of EMT and stem cell properties in therapy survival mechanisms. 
A.

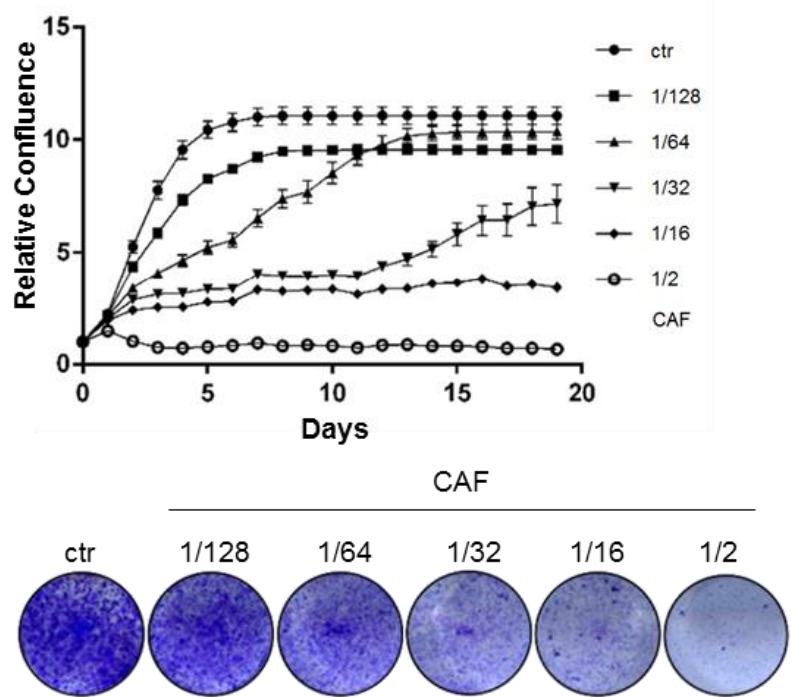

B.

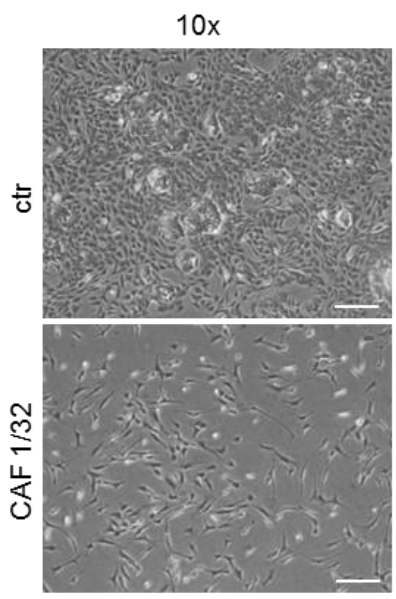

C.

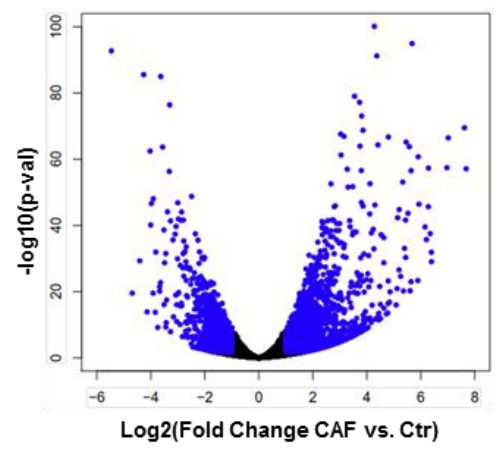

D.

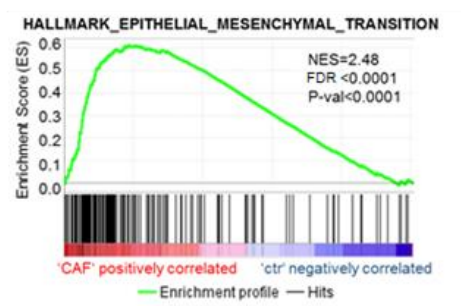

ANASTASSIOU MULTICANCER INVASIVENESS_SIGNATURE

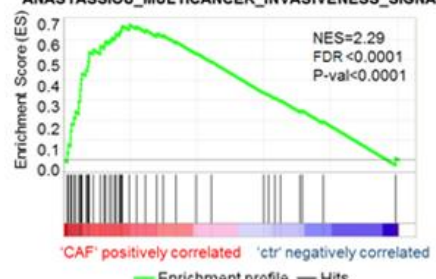

Enrichment profile - Hits

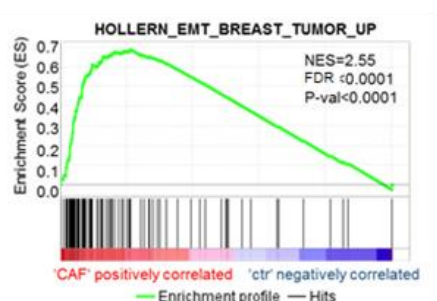

LM MAMUARY STEM_CELL_UP

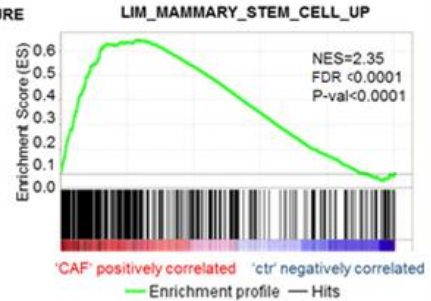

E.

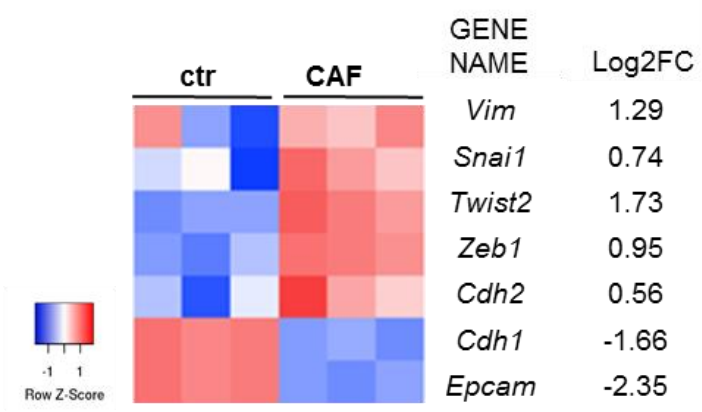

F.

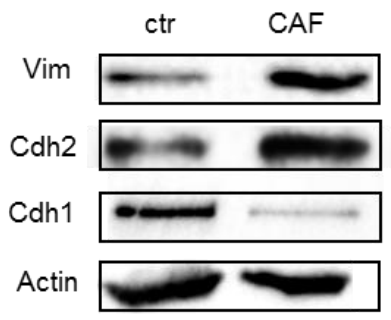

G.
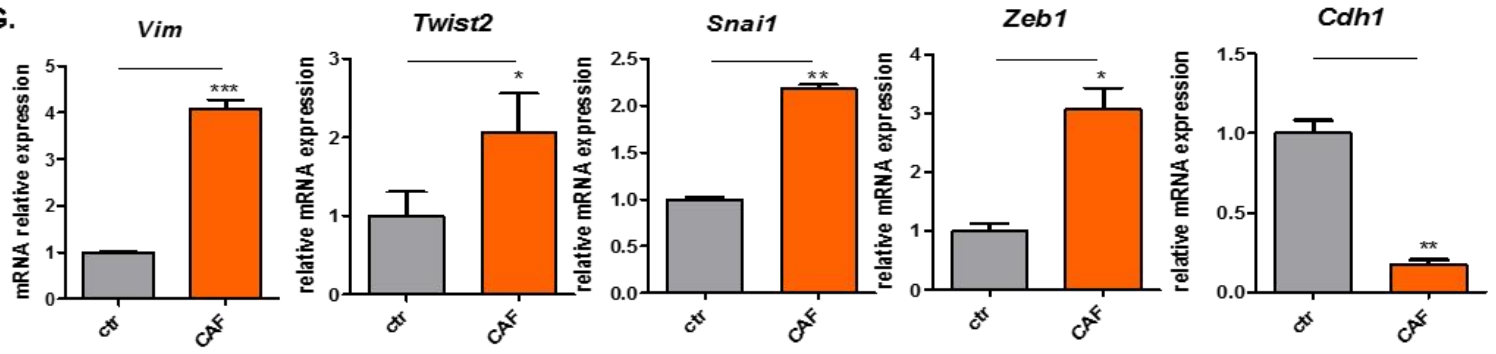
Fig. I 1 WAP-T cells surviving CAF treatment gain stem cell and EMT properties in vitro. A: Cell proliferation assay of pG-2 cells treated for 48 hours with increasing concentrations of a combinatory CAF chemotherapy. The concentration [1] represents the equivalent of the doses used in previous in vivo experiments $(10 \mu \mathrm{g} / \mathrm{ml}$ cyclophosphamide, $0.5 \mu \mathrm{g} / \mathrm{ml}$ doxorubicin and $10 \mu \mathrm{g} / \mathrm{ml} 5$-FU) (Jannasch et al., 2015). Cell confluency was assessed every day using Celigo. For a direct visualization, crystal violet staining was performed at day 2. B: Phase contrast images of pG-2 cells after 48 hours CAF-treatment showing a spindle like morphology characteristic for cells that underwent EMT (objective $10 \mathrm{x}$, scale bar $=250 \mu \mathrm{m}$ ). C: Volcano plot showing transcriptome wide gene expression changes in $\mathrm{pG}$ 2 cells compared to $p G-2$ cells after 48 hours of CAF-treatment, as measured by RNA-seq ( $n=3$ biological replicates). D: Representative GSEA enrichment plots showing a significant enrichment of gene signatures characteristic for EMT-processes, stemness traits and cancer invasiveness in CAF-treated versus control cells. E: Heatmap showing the regulation of selected EMT markers identified in the RNAseq analyses. $\mathbf{F}$ and $\mathbf{G}$ : Validation of EMT-marker regulation on protein level using western blot $(\mathbf{F})$ and on mRNA level using qRT-PCR $(\mathbf{G})$. qRT-PCR data was normalized to the control condition and normalized to the Rplpo. $\mathrm{n}=3$ biological replicates $+/-\mathrm{SEM},{ }^{*} \mathrm{p}$-val $\leq 0.05,{ }^{* *} \mathrm{p}$-val $\leq 0.01,{ }^{* * *} \mathrm{p}$-val $\leq$ 0.005 .

\section{WAP-T tumor cells surviving CAF-treatment downregulate the expression of PRC2 core subunits}

We decided to get more insight into the molecular mechanisms allowing tumor cells to activate EMT and stemness transcriptional programs, increasing their aggressiveness and survival to cytotoxic therapies. We therefore returned to our GSEA analyses and interestingly identified an accumulation of gene signatures related to epigenetic regulatory pathways perturbation enriched CAF-treated cells (Fig. I 2A). This was an interesting finding, as several epigenetic mechanisms have been shown to be involved in the processes controlling cellular plasticity (Kiesslich, Pichler, \& Neureiter, 2012). Based on the RNA-seq results, we identified 65 down-regulated and 16 upregulated epigenetic factors (Fig. I 2B, listed in Table I S1). Surprisingly, Gene Set Enrichment Analysis (GSEA) and Enrichr analyses pointed at an enrichment of genes known to be H3K27me3-marked and/or repressed by PRC2 (Fig. I 2C). We therefore checked if changes of PRC2 subunits expression happened upon chemotherapy treatment. Strikingly, the core PRC2 subunits Ezh2, Suz12, Rbpp7 were found to be significantly downregulated in cells surviving the CAF treatment (Fig. I2D). The downregulation of Ezh2, Suz12 and Rbbp7 was validated by qPCR (Fig. I 2E). On protein level, Ezh2 and Suz12 were reduced as assessed via western blots and immunofluorescence staining (Fig. I 2F-G). In line with these findings, rG-2 cells grown under normal conditions harbored a constant lower expression of the core PRC2 subunits Ezh2, Suz12 and Rbbp7 when compared to untreated or treated pG-2 cells. 
Noticeably, their expression levels were even more reduced upon CAF treatment (Fig. I S2). We concluded that the reduction of PRC2 level was associated with survival to cytotoxic therapies and with increased resistant phenotype. 
A.

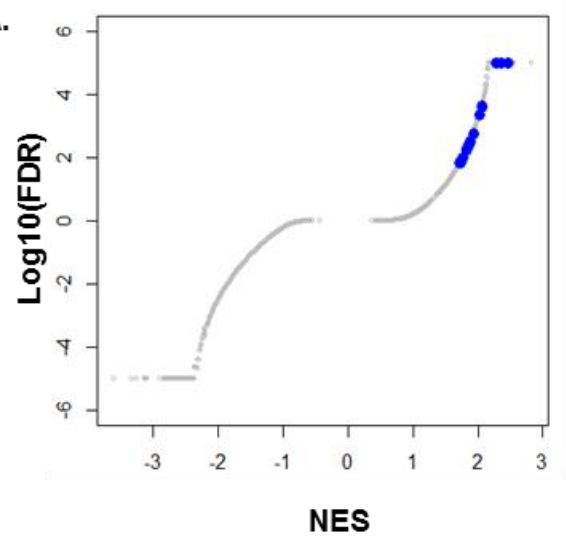

B.

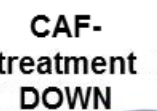

CAF.

treatment

UP

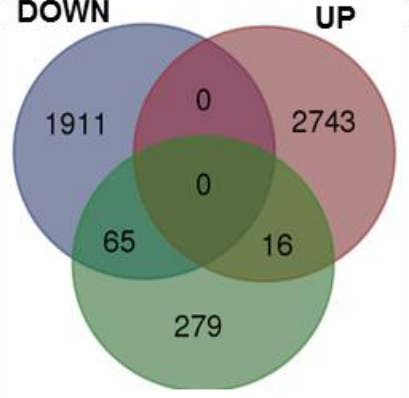

Epigenetic factors

c.

D.

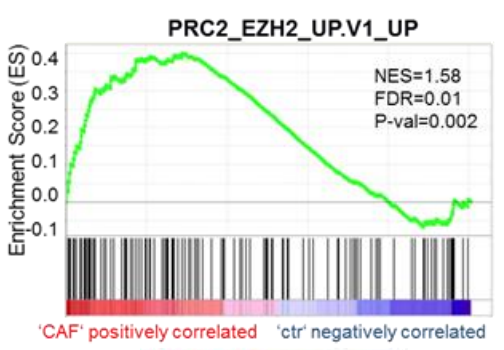

- Enrichment profile - Hits

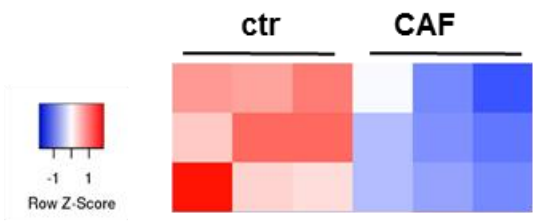

E.

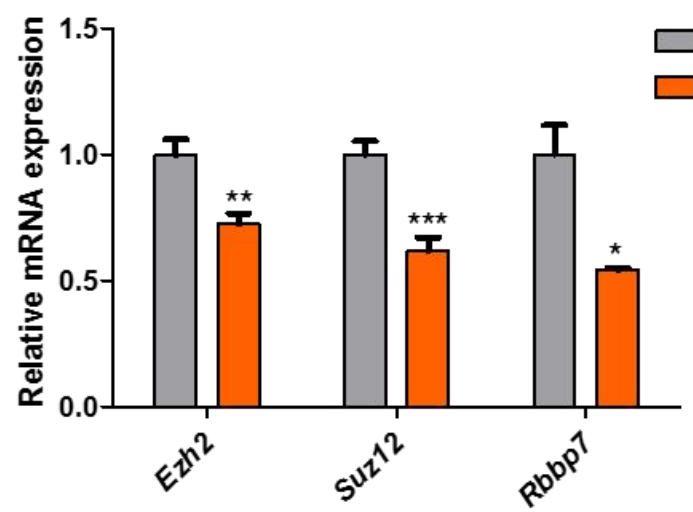

PRC2_SUZ12_UP.V1_UP

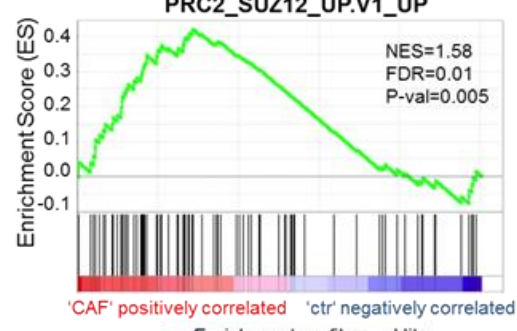

- Enrichment profile — Hits

GENE

NAME Log2(FC)

Ezh2 $\quad-0.77$

Suz12 -1.70

Rbbp7 $\quad-0.96$

G.

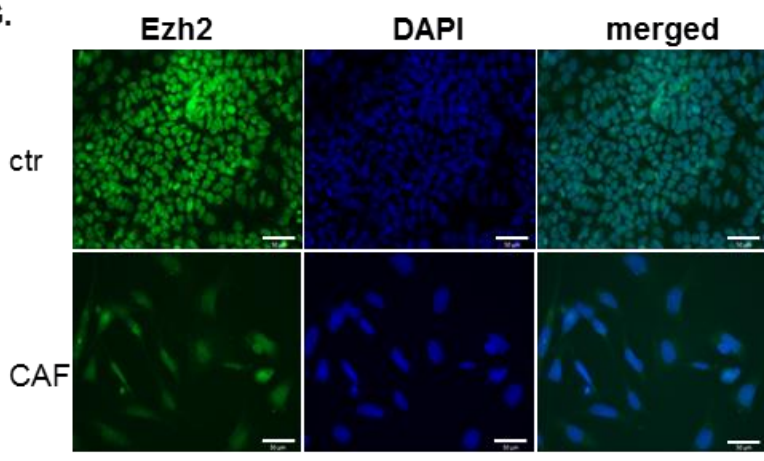

F.

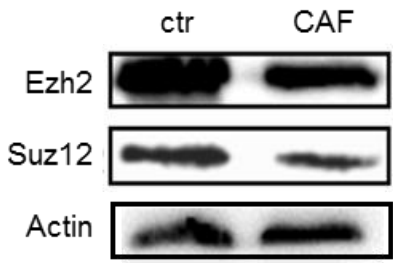


Fig. I 2 WAP-T tumor cells surviving CAF treatment downregulate the expression of PRC2 core subunits. A: GSEA analysis results (MSigDB) plotted as an overview along Normalized Enrichment Score (NES) and log10(FDR). The results show an enrichment of gene signatures associated with epigenetic mechanisms perturbation. Blue dots represent enriched epigenetic pathway. B: Identification of differentially regulated epigenetic factors: genes regulated in $\mathrm{pG}-2$ cells upon CAF treatment survival (|Log2(Fold Change) $\mid>0.8$, padj<0.05) were intersected with a list of known epigenetic factors. C: Representative GSEA enrichment plots showing the enrichment of gene signatures typically repressed by PRC2 in CAF-treated pG-2 cells. D: Heatmap showing the downregulation of central PRC2 members upon chemotherapy, as identified in the RNA-seq analyses, padj<0.05. E: Validation of Ezh2, Suz12 and Rbbp7 expression via qRT-PCR. Data was normalized on the control condition and normalized to the Rp/p0 housekeeping gene. $\mathrm{n}=3$ biological replicates + - SEM, ${ }^{*} \mathrm{p}$-val $\leq 0.05,{ }^{* *} \mathrm{p}$-val $\leq 0.01,{ }^{* * *} \mathrm{p}$-val $\leq$ 0.005.F and G: Reduction of EZH2 and SUZ12 protein levels upon CAF treatment was assessed via western blot $(\mathbf{F})$ and immunofluorescence staining $(\mathbf{G})$.

\section{Reduction of EZH2 activity enhances the aggressiveness of TNBC tumor cells}

Although the majority of the literature attributes rather tumor promoting functions to the PRC2 complex, a few recent publications have pointed towards a possible tumor suppressive role in ovarian carcinoma (Cardenas et al., 2016). We therefore asked whether the reduction of PRC2 activity could directly mediate WAP-T tumor cell survival to cytotoxic therapies by derepressing aggressive and/or proliferative gene expression programs. To assess the effect of EZH2 activity loss on the proliferation of pG-2 cells, we silenced Ezh2 using targeted siRNA or treated the cells with a small molecule inhibitor against EZH2 (EPZ-6438) and performed proliferation assays. Interestingly, impairment of EZH2 activity did not reduce proliferation of the tumor cells as it was observed for numerous other BC cell lines in the past (Gonzalez et al., 2009; Song et al., 2016). On contrary, the growth of pG-2 cells was slightly but significantly promoted upon EZH2 knockdown (Fig. I 3A) and low concentrations of EPZ-6438 (Fig. I 3D). Ezh2 knockdown efficiency was validated at mRNA level (Fig. I 3B) and loss of H3K27me3 resulting from EPZ-6438 treatment was measured by western blot for different concentrations (Fig. I 3C). Interestingly, colony formation ability of pG-2 cells seeded at limiting dilution was strongly improved upon inhibition of EZH2, suggesting increased tumor initiating properties (Fig. I 3E). Strikingly, this increased colony formation capacity was maintained upon chemotherapy treatment, indicating that the inhibition of the PRC2 complex activity indeed supported cell survival and resistance to the therapy (Fig. I 3E). We asked whether this observation was limited to the murine WAP-T mammary carcinomas or if other human cancer cell lines could also get a growth and survival advantage upon PRC2 activity loss. Interestingly, although certain breast 
cancer cell lines showed impaired or unchanged proliferation upon EZH2 inhibition, the MDA-MB-468 TNBC cell line displayed moderate but increased growth properties when treated with siRNA against EZH2 or with EPZ-6438 (Fig. I S3). Interestingly, the proliferation stimulating consequence of an EZH2 inhibition was not limited to breast cancer cell lines, but was also observed in human cancer cell lines of other origins, colorectal and bile duct carcinoma (Fig. I S4). Moreover, the proliferation advantage mediated by EZH2 knockdown in MDA-MB-468 was even more pronounced in the presence of CAF treatment (Fig. I S3B). Together, inhibition of PRC2 repressive activity increases aggressiveness of cancer cells and increases cytotoxic therapy survival in a context dependent manner. 
A.

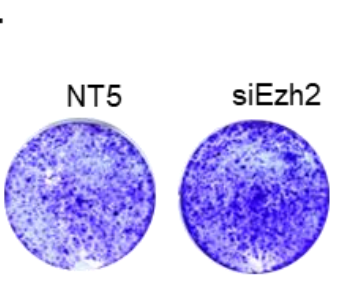

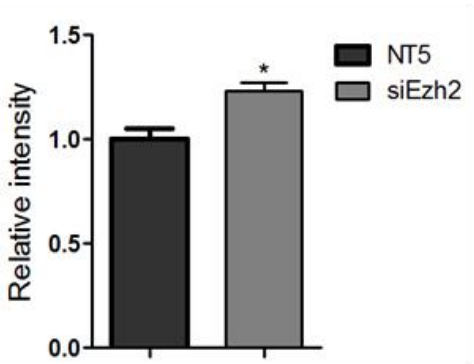

B.

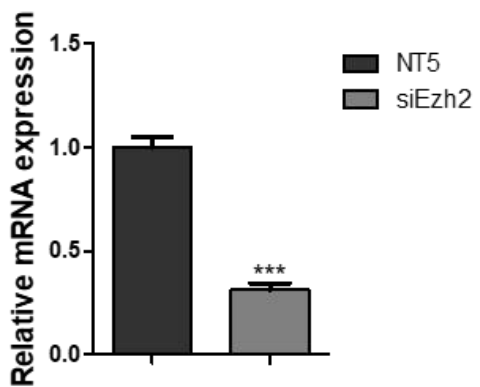

c.
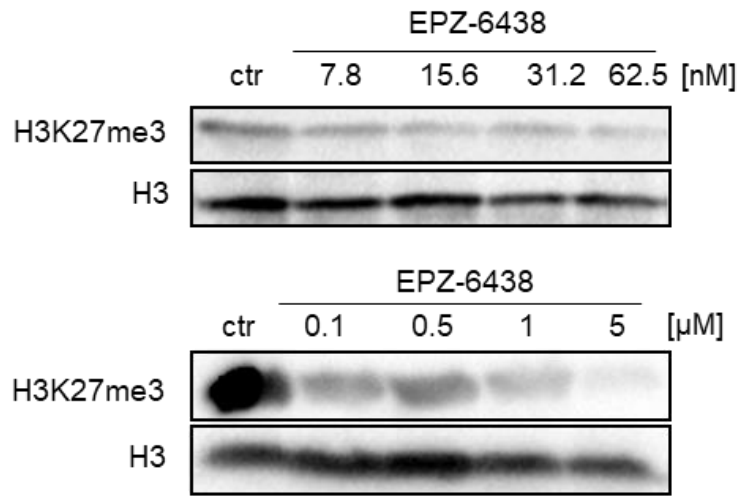

D.

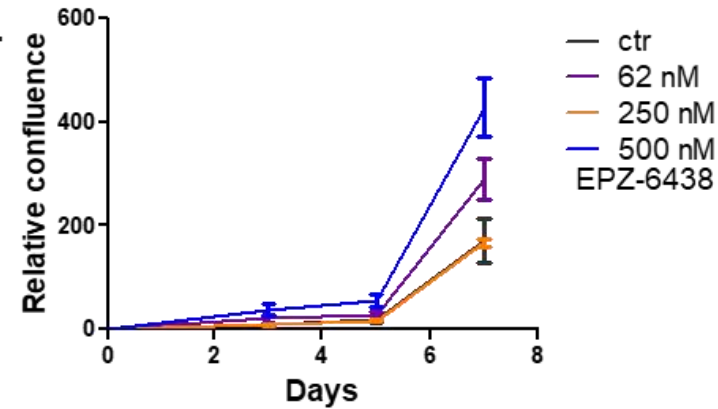

E.
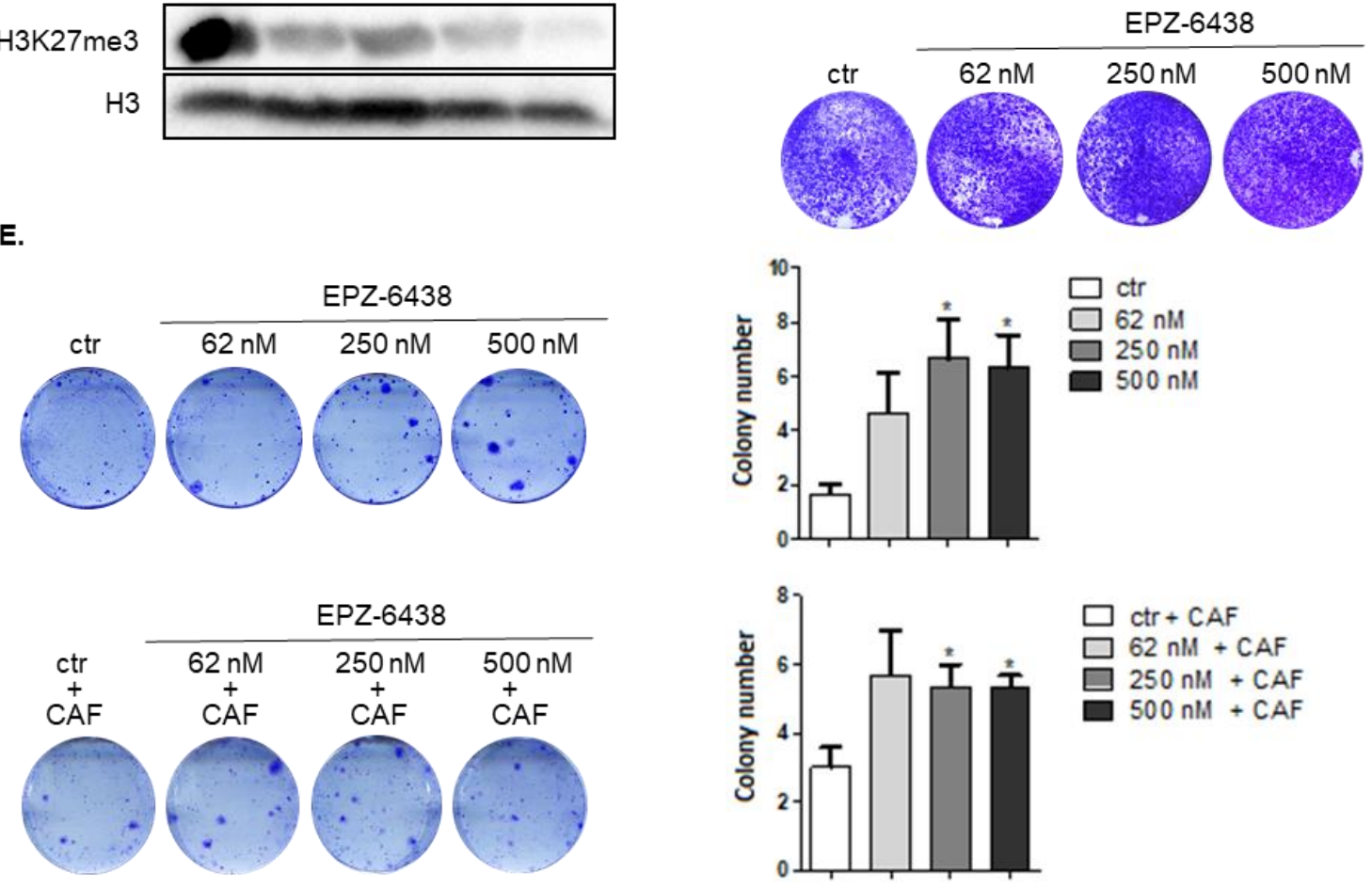

Fig. I 3 Reduction of EZH2 activity enhances the aggressiveness of TNBC tumor cells.

A: Crystal violet staining of pG-2 cells upon Ezh2 knockdown. The confluency was measured by ImageJ and normalized to the controls. B: Validation of Ezh2 knockdown efficiency using qRT-PCR. Data were calibrated to the control condition and normalized on the Rp/pO. C: Assessment of EZH2 inhibition by H3K27me3 levels upon increasing EPZ-6438 concentration using western blot. D: Proliferation assay of EPZ-6438-treated pG-2 cells using Celigo ${ }^{\circledR}$ and crystal violet staining. E: Colony formation assay upon treatment of pG-2 cells with EPZ-6438 alone or in combination with CAF. Number of colonies were assessed through ImageJ analysis. $n=3$ biological replicates $+/$ - SEM, ${ }^{*} p$-val $\leq 0.05,{ }^{* *} p$-val $\leq 0.01,{ }^{* * *}$ $\mathrm{p}$-val $\leq 0.005$. 
Reduction of PRC2 activity during chemotherapy treatment enables the activation of gene expression programs promoting tumor cell survival.

The trimethylation of $\mathrm{H} 3 \mathrm{~K} 27$ by PRC2 mediates the silencing of chromosomal regions by promoting chromatin compaction through cooperation with the PRC1 complex (Grossniklaus \& Paro, 2014). Furthermore, because of the occupancy of the presence of the methyl groups, $\mathrm{H} 3 \mathrm{~K} 27 \mathrm{me} 3$ is mutually exclusive with the transcriptional activating mark H3K27ac (Tie et al., 2009). Relying on this knowledge, we hypothesized that loss of PRC2 activity during chemotherapy survival could lead to an epigenetic switch enabling tumor cells to activate translational programs promoting aggressiveness and therapy resistance. To test our hypothesis, we assessed genome wide changes of H3K27me3 and H3K27ac occupancy via ChIP-seq in untreated as well in 48 and 96 hours CAF-treated pG-2 cells. Because we suspected a direct connection between PRC2 repressive activity loss and activation of genes expression programs upon $\mathrm{CAF}$ treatment, we decided to investigate the changes of $\mathrm{H} 3 \mathrm{~K} 27 \mathrm{me} 3$ and H3K27ac at the TSS region of upregulated genes. As presumed, the levels of H3K27me3 at promoter regions of up-regulated genes were significantly reduced already after 48 hours and remained low after 96 hours (Fig. I 4A). This analysis uncovered a switch from $\mathrm{H} 3 \mathrm{~K} 27 \mathrm{me} 3$ to $\mathrm{H} 3 \mathrm{~K} 27 \mathrm{ac}$ indicating potential genes activated through PRC2/EZH2 loss. H3K27ac as a mark of active promoters and active gene transcription, we observed its increased level upon chemotherapy. In this analysis, 74 genes showed at the same time a robust up-regulation at the RNA level (Log2FC $>0.8$, p-val < 0.05) and a switch from trimethylation to acetylation at H3K27 (Fig. I 4B). Moreover, we identified a number of upregulated genes with subsequent loss of H3K27me3 and H3K27ac gain (Fig. I 4C). We selected few of them with the most relevant H3K27me3/H3K27ac change at promoter regions, such as Nfatc1, Wnt9a, Gli2 and Klf4 (Fig. I 4C, D). Additionally, RNA-seq results presented on the heatmap, shows a characteristic upregulation of the aforementioned genes (Fig. I 4D). To investigate enrichment signatures between CAF-treated (48h) and control cells, we used the online Enrichr tool (Fig. I 4E). One of the most significantly enriched pathways, was calcineurin signaling involving NFATc1. NFATc1 activation was shown to promote EMT and tumor 
progression in several tumor entities. Furthermore, Chen et al. reported a context dependent epigenetic regulation of NFATc1 expression by EZH2 in pancreatic tissues (N. M. Chen et al., 2017). Additionally, NFATc1 can be targeted by small molecule inhibitors, some of them being commonly employed in the clinic (e.g. Cyclosporin A, CsA), making this factor very attractive to study in the context of survival and resistance to chemotherapy (Pan, Xiong, \& Chen, 2013). Upregulation of Nfatc1 upon CAFchemotherapy treatment was confirmed on mRNA (Fig. I 4F) and protein level (Fig. I $4 G$ ) indicating its potential role in cancer recurrence. 
A.

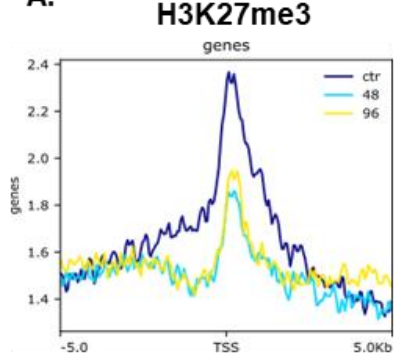

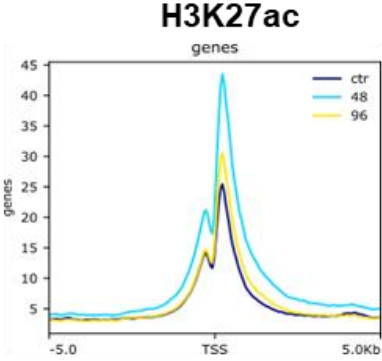

B.

H3K27me3 loss and

H3K27ac gain

(ChIP-seq)

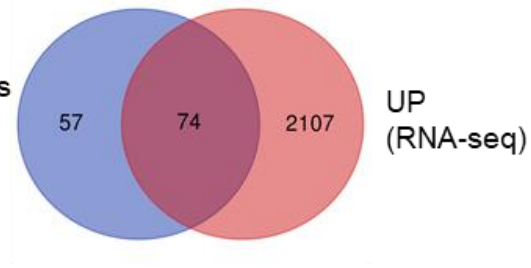

C.

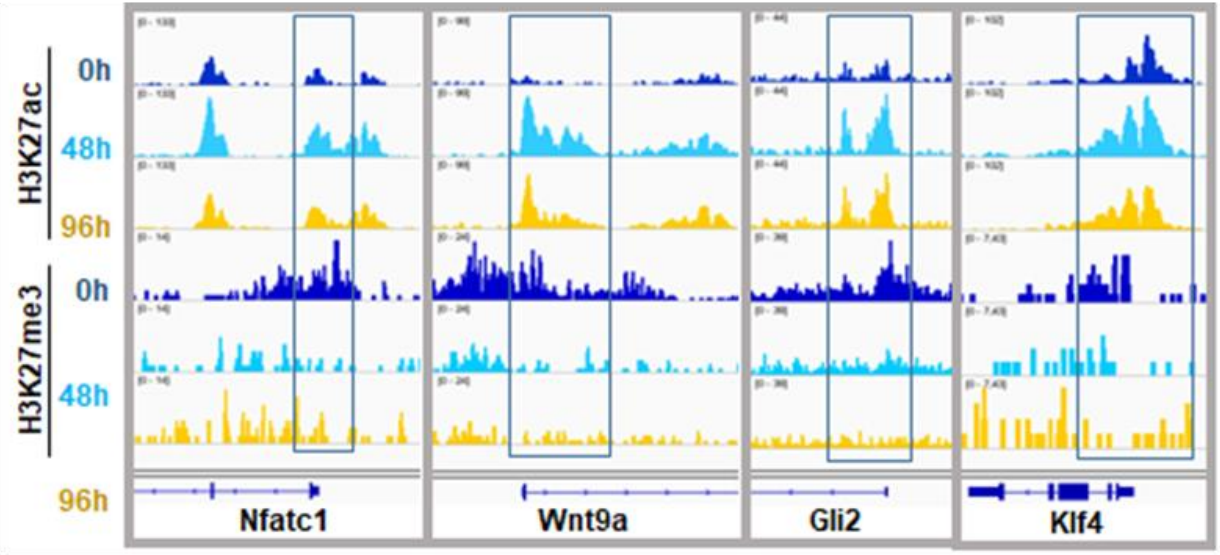

D.

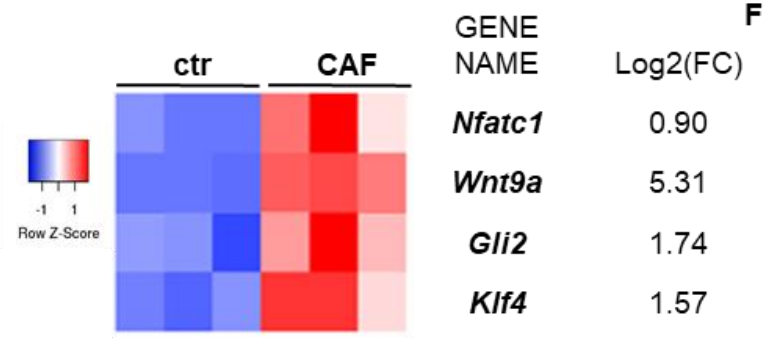

F.

Nfatc1

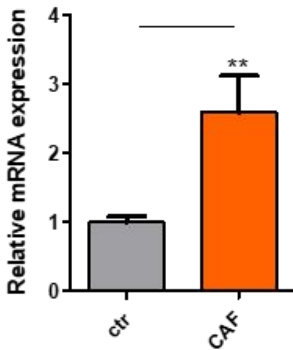

G.

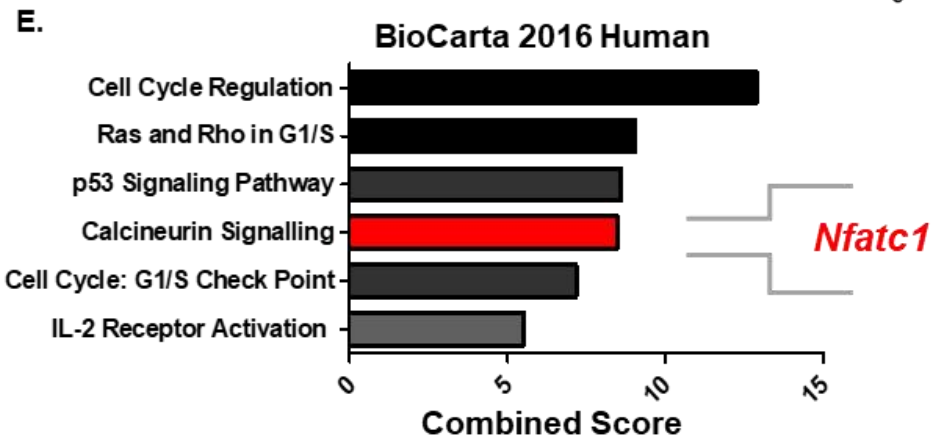

Fig. I 4 CAF-chemotherapy-induced epigenetic regulation in pG-2 cells. A: Aggregate plots of H3K27me 3 and H3K27ac ChIP-seq signals at $\pm 5 \mathrm{~kb}$ of the TSS) of genes that were upregulated (RNAseq) upon CAF-chemotherapy treatment for 48 hours (48, light blue line), treated with CAFchemotherapy for 48 hours and fresh medium for the next 48 hours (96, dark blue line) and untreated (ctr, yellow line). B: Venn diagram showing the overlap of the H3K27me3 loss and the H3K27ac gain ChIP-seq peaks with upregulated genes, $0.8 \leq \log 2(\mathrm{FC})$, from RNA-seq data. C: H3K27me3 and H3K27ac ChIP-seq tracks at Nfatc1, Wnt9a, Gli2 and Klf4 gene loci in CAF-chemotherapy treated (48h, 
96h) and control cells (0h). H3K27me loss and H3K27ac gain at promoter region, indicated in blue boxes D: RNA-seq heatmap of selected upregulated genes upon 48 hours CAF treatment. E: Enrichr analysis, BioCarta for the most significant pathways, on the 74 identified genes from Fig. I 4B. F: Expression of Nfatc1 on mRNA, +/- SEM, ${ }^{*} p$-val $\leq 0.05,{ }^{* *} p$-val $\leq 0.01,{ }^{* * *} p$-val $\leq 0.005$ and G: protein level in 48 hours CAF-treated pG-2 cells.

\section{EZH2 loss mediates NFATc1-induced cancer progression in TNBC}

To investigate whether EZH2 modulates NFATc1 expression, we performed knockdown of Ezh2 in pG-2 cells. Upon Ezh2 loss, we observed a decrease in H3K27me3 and a Nfatc1 upregulation, meaning that EZH2 activity negatively modulates NFATc1 expression (Fig. I 5A). To further analyze Ezh2 and Nfatc1 expression in vivo, we used WAP-T mice tumors followed by CAF treatment (Fig I 5B). $\mathrm{IHC}$ staining revealed a loss of EZH2 and an upregulation of NFATc1 in the acute phase of CAF treatment. Whereas during the recovery phase, Ezh2 expression increased and Nfatc1 level came back close to the basal level (control) indicating rapid changes in gene expression upon cytotoxic stimuli. Based on human primary breast tumors, gene expression signature analysis also suggests a frequent negative correlation between EZH2 and NFATC1 (Fig. I 5C). Additionally, we used The Cancer Genome Atlas (TCGA) PAM50-based database for human TNBCs where we could observe differential EZH2, NFATC1 expression among patients (Fig. I5D). Interestingly, survival plots using patients data with low or high expression of EZH2 (left) and NFATc1 (right) (Fig. I 5E) suggest that patients survival is positively correlated with EZH2 expression and negatively correlated with NFATc1. These data suggest that NFATc1 can be a potential anticancer target in TNBC. 
A.

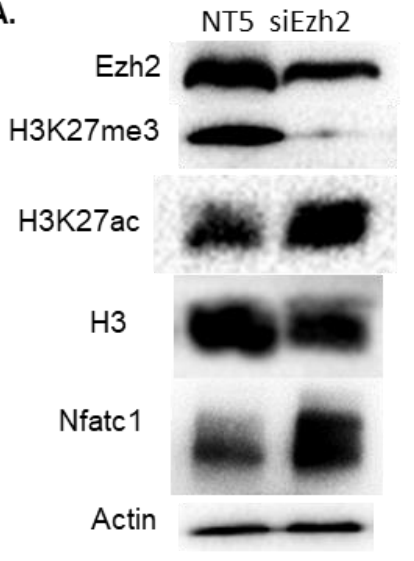

C.

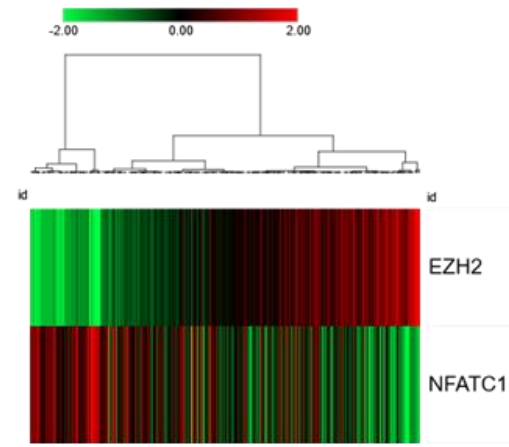

E.
D.

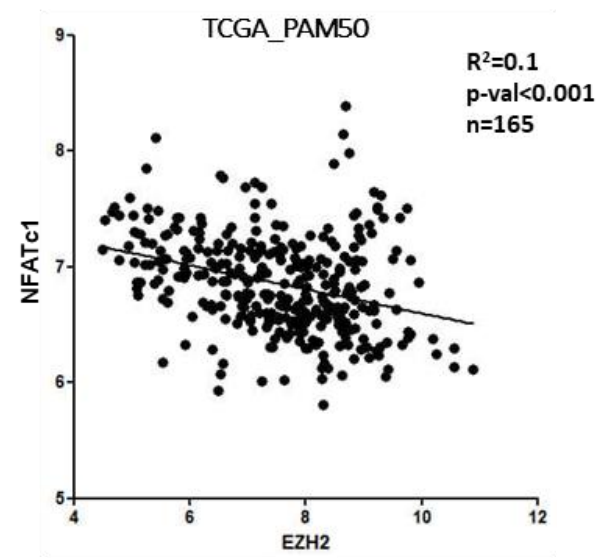

Group 3

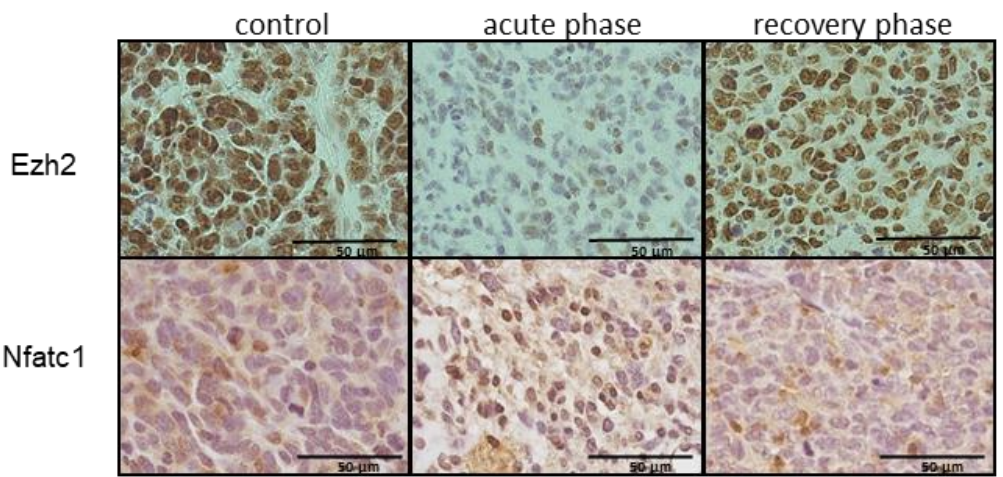

\section{Survival of EZH2_TCGA_PAM50}

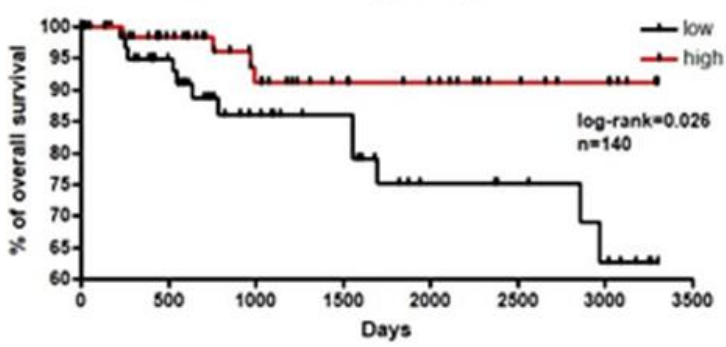

Survival of NFATC1_TCGA_PAM50

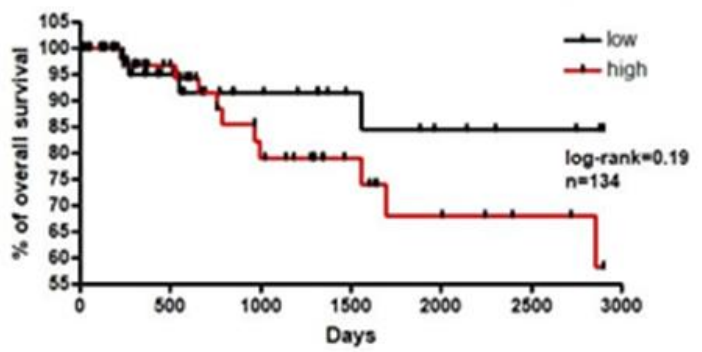

Fig. I 5 Differential expression of Ezh2 and Nfatc1 in TNBC. A: Nfatc1 regulation upon loss of EZH2 in protein level in pG-2 cells. B: Representative images of paraffin-embedded tumors from Group 1: dissected when tumors reached $0.5 \mathrm{~cm}^{3}$, Group 2: CAF treated and dissected after 9 days and Group 3: CAF treated and dissected when reaching initial tumor volume, stained for Ezh2 and Nfatc1. C: Gene expression signature of Ezh2 and Nfatc1 using MERAV presented with Pearson correlation that is -0.21 in primary breast tumors. D: TCGA PAM50 (Xenabrowser)-based scatter plot with linear regression of the genome-wide correlation between EZH2 and NFATC1. E: TCGA PAM50-based survival of TNBC patients based on EZH2 and NFATc1 expression level; low or high. 


\section{NFATC1 knockdown decreases TNBC cell invasiveness}

NFAT proteins have been shown to be involved in EMT processes in breast cancer (Sengupta et al., 2013). In this part of the study we wanted to verify NFATc1 function in TNBC and investigate its involvement in cancer cell motility, growth, EMT and stemness. Nfatc1 knockdown decreased pG-2 cell growth (Fig. I 6A, B), where Nfatc1 loss efficiency was confirmed on mRNA (Fig. I 6C) and protein level (Fig. I 6D). Additionally, we observed great proliferation impairment upon NFATC1 depletion alone or in combination with CAF in the human breast cancer cell line, MDA-MB-468 (Fig. I S5). In pG-2 cells, Nfatc1 loss lead to reduced migratory ability (Fig. I 6E, F). Based on the findings on epithelial-mesenchymal plasticity (EMP) of metastatic breast cancer and our previously shown data (Fig. I 1), we analyzed EMT-related markers in the context of Nfatc1 regulation. Our data demonstrate that upon loss of Nfatc1, TNBC cells change to a more epithelial phenotype (Fig. I G, H). In addition, we treated pG-2 cells with cyclosporine $A(C s A)$ and Thapsigargin to inhibit and stimulate Nfat activity, repectively (Fig. I 6H, Fig. I S6). The cells were treated with increasing concentrations of CsA and Thap for 48 hours, stained with FITC conjugated antibody against EpCAM. We observed an increase and a decrease in EpCAM-positive cells upon CsA and Thap treatment respectively, suggesting a key role of Nfatc1 in EMT and indicating its involvement in cancer cell survival. 
A.

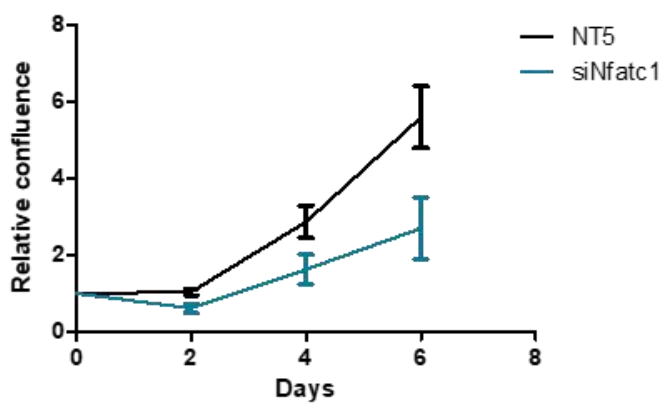

E.
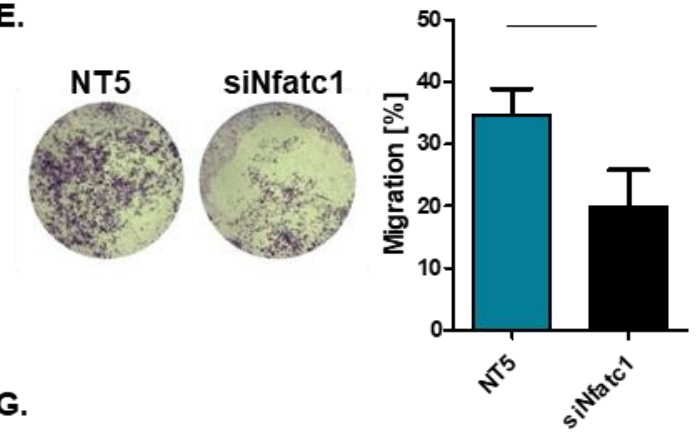

G.
B.

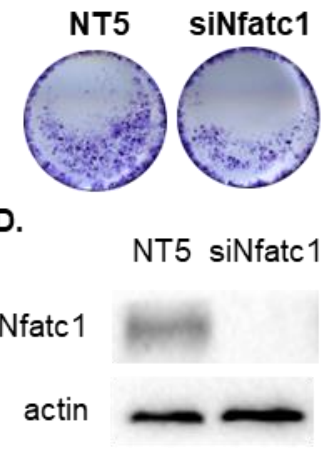

F.

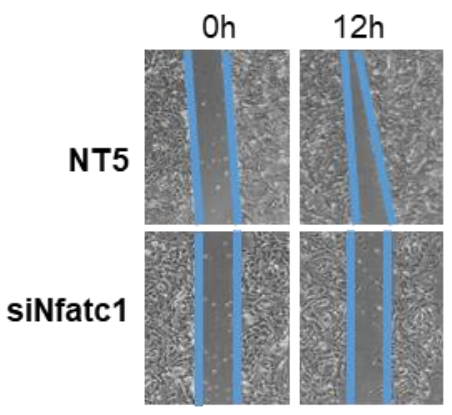

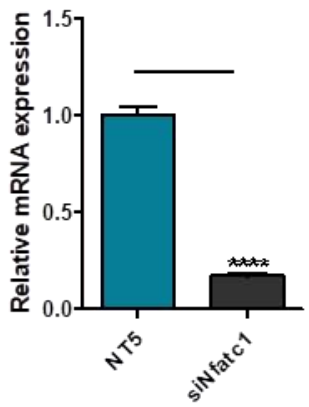

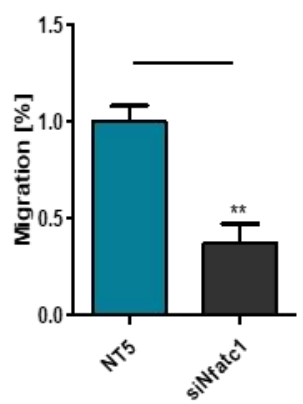

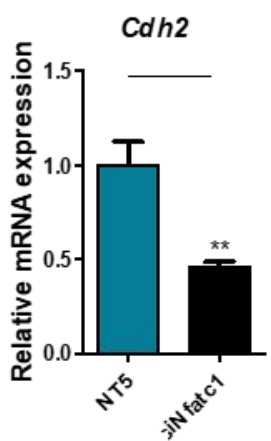
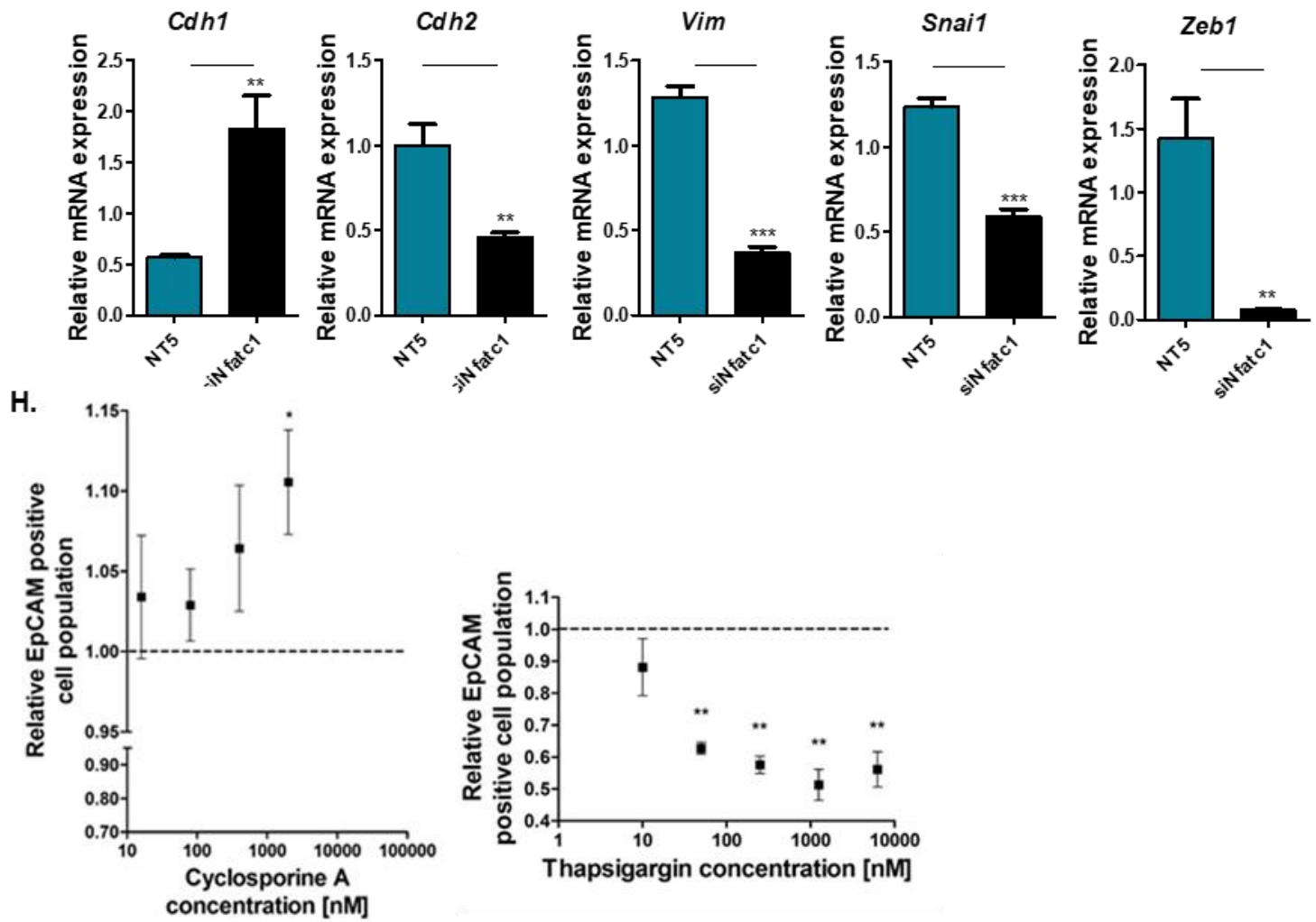

Fig. I 6 Nfatc1 inhibition and knockdown abrogates oncogenic properties of TNBC cells. A: Suppression of cell growth upon Nfatc1 knockdown determined by Celigo and B: crystal violet. C: Knockdown of Nfatc1 was validated on mRNA and D: protein level. E: Reduced migration capacity upon Nfatc1 knockdown assesed using Boyden chamber assay and F: scratch assay. G: Regulation of EMT markers upon Nfatc1 knockdown on mRNA level, $n=3+/$ - SEM, ${ }^{*} p$-val $\leq 0.05,{ }^{* *} p$-val $\leq 0.01,{ }^{* * *} p$-val $\leq$ 
0.005. H: FACS showing an increase of EpCAM positive cells upon inhibition of Nfatc1 via cyclosporine A treatment and decrease of EpCAM positive cells followed by Nfatc1 activation upon thapsigargin treatment.

\section{Discussion}

In contrast to early and locally constrained breast cancer, advanced metastatic disease is often considered as incurable (Harbeck et al., 2019). For this reason, therapies of cancers in advanced stages mainly focus on patient survival and life quality improvement. Because of the lack of specific therapeutic targets, treatment of advanced TNBC almost exclusively relies on the efficiency of cytotoxic therapies, while also being prone to resistance. Hence, a better understanding of the mechanisms leading to chemotherapy resistance represents a crucial step for the development of more efficient therapeutic approaches. In the present study, we utilized the murine WAP-T mammary carcinoma cell system to model and investigate molecular mechanisms underlying TNBC survival to conventional chemotherapy. Our transcriptome wide analyses showed that WAP-T cells activate transcriptional programs characteristic for EMT and cancer stem cells during survival to treatment. Interestingly, gain of epithelial-mesenchymal plasticity was shown to promote tumor cells invasiveness and protect them from pro-apoptotic signals (Kalluri \& Weinberg, 2009; Scheel \& Weinberg, 2011). Additionally, EMT and CSC properties are tightly linked together and have been frequently shown to positively influence each other (Hennessy et al., 2009; Loret, Denys, Tummers, \& Berx, 2019; Mani et al., 2008). Notably, both EMT and CSC properties were implicated in the acquisition of chemotherapy resistant phenotypes by the tumor cells (Izumiya et al., 2012). In order to develop resistance, cell have to rapidly and profoundly reorganize their transcriptional programs. Therefore, because of their very dynamic nature, we expected epigenetic mechanisms to be involved. Combining mRNA-seq and ChIP-seq approaches, we identified a reduction of the PRC2/EZH2 activity occurring during chemotherapy survival in WAP-T cells. Interestingly, the repressive activity of EZH2 on gene expression is mostly known to promote cancer progression and contribute to therapy resistance in various types of cancer (Hirukawa et al., 2018; Hu et al., 2010; Kikuchi et al., 2015; Zhang et al., 2013). Specifically, EZH2 activity was implicated in resistance to programmed cell death in TNBC (J. P. Huang \& Ling, 2017; P. Zhang et 
al., 2018). Paradoxically, our results unraveled an opposite role of PRC2/EZH2 in TNBC cells, maintaining a more chemotherapy sensitive phenotype via specific repression of EMT and CSC transcriptional programs. Although apparently contradictory at the first glance, our results align with still scarce but growing evidences, that loss of PRC2/EZH2 activity can drive or support initiation and progression of cancers in a context specific manner (Vo et al., 2017; Wassef et al., 2015). In 2015, the group of Raphaël Margueron elegantly demonstrated that reduced EZH2 expression promotes transcriptional instability and is likely to promote breast tumorigenesis (Wassef et al., 2015). Shortly later, Serresi and colleagues showed in two consecutive studies on Non-Small-Cell-Lung Cancer that PRC2 activity can act as a barrier to KRAS-driven inflammation and EMT (Serresi et al., 2016). Our investigations on murine and human TNBC cell lines corroborated these observations and described thereby a new molecular mechanism by which PRC2/EZH2 can exert its repressive function on the EMT transcriptional program. Specifically, loss of PRC2 subunits upon chemotherapy treatment leads to a rapid upregulation of central EMT regulators via a repressive (H3K27me3) to activating (H3K27ac) epigenetic switch. Strikingly, we identified here NFATC1 as one of the major EMT-TF under the immediate epigenetic control of PRC2 in TNBC and upregulated in cells surviving chemotherapy. Interestingly, the group of Hessmann reported that NFATc1 is needed for pancreas during regeneration after injury and is epigenetically silenced by EZH2 activity once regeneration is completed, supporting the mechanism of regulation identified in the present study (N. M. Chen et al., 2017). The pivotal role of NFATc1 in the activation of EMT transcriptional programs in cancer cells and the availability of specific small molecule inhibitors (e.g. cyclosporine A or VIVIT) renders this factor a very interesting potential drug target to increase conventional therapies efficiency (Aramburu et al., 1999; F. Liu et al., 2009). In this study, we observed an increased efficiency of CAF treatment on TNBC cells when co-treated with cyclosporine A or VIVT. These results are in line with former studies on lung cancer, acute myeloid leukemia (AML) and bladder cancer showing that NFATc1 inhibition sensitized cancer cells to cisplatin, sorafenib- and tacrolius-induced apoptosis, respectively (Im et al., 2016; Kawahara et al., 2015; Metzelder et al., 2015). 


\section{Conclusions}

This study presents the evidence of a context dependent PRC2/EZH2 function in breast cancer that in certain circumstances can sensitize the cells to chemotherapy by epigenetically repressing NFATc1 expression. Our data suggests that targeting NFATc1 signaling in TNBC patients with low EZH2 expression could increase the efficiency of conventional chemotherapeutic treatments and reduce the development of resistance. 


\section{Supplementary figures}

A.
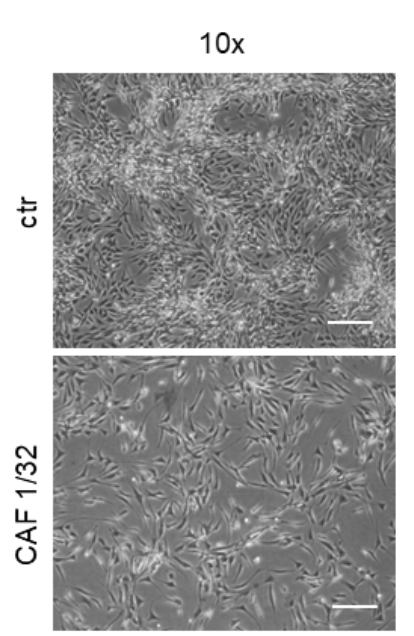

B.
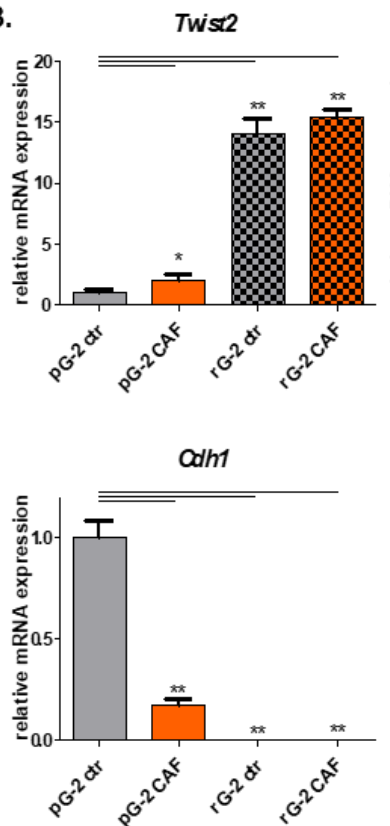
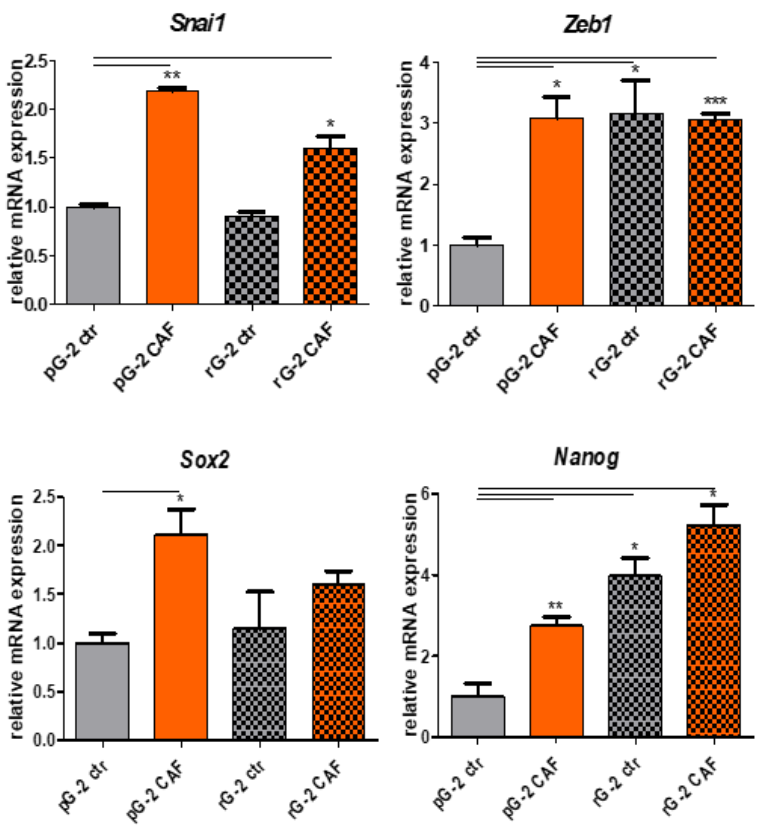

Fig. I S1 WAP-T cells surviving CAF treatment with EMT and stemness changes in vitro. A: Phase contrast images of rG-2 cells 48 hours after CAF-treatment show (objective 10x, scale bar $=250 \mu \mathrm{m}$ ). B: EMT markers expression on mRNA level in pG-2 and rG-2 cells, $\mathrm{n}=3$, normalized to Rplp0, +/- SEM, ${ }^{*} \mathrm{p}$-val $\leq 0.05,{ }^{* *} \mathrm{p}$-val $\leq 0.01,{ }^{* * *} \mathrm{p}$-val $\leq 0.005$.

A.

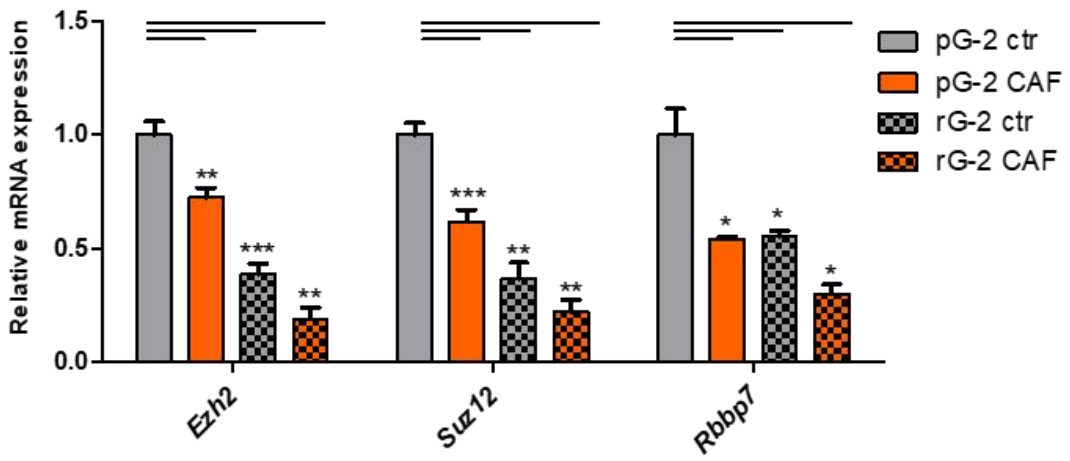

B.
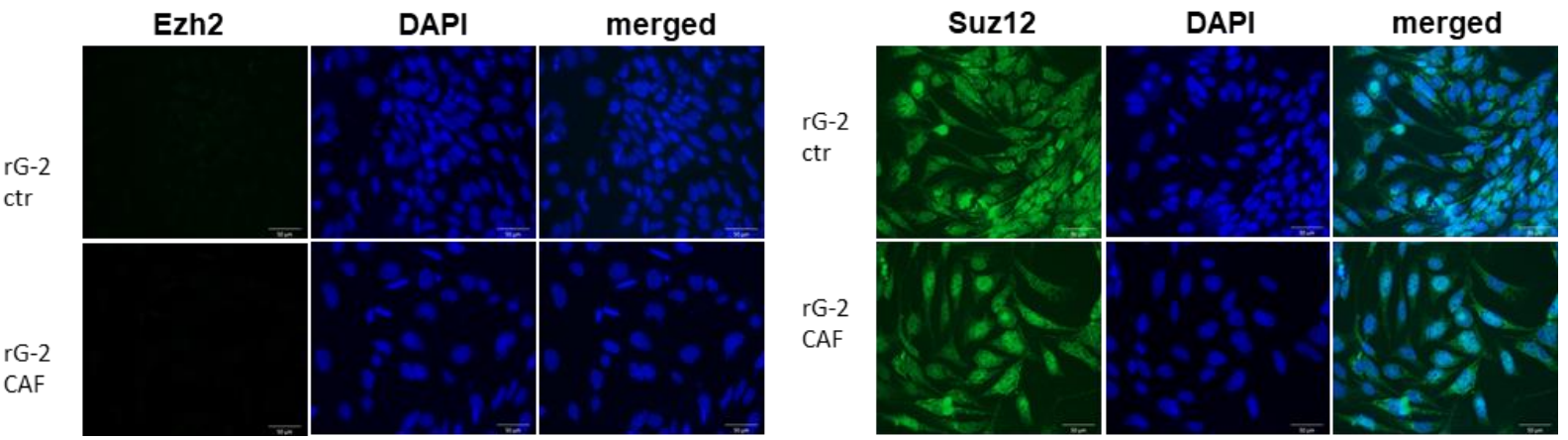

Fig. I S2 Regulation of PRC2 members in pG-2 and rG-2 cells upon 1/32 chemotherapy treatment. A: Relative mRNA expression of Ezh2, Suz12 and Rbbp7 upon $48 \mathrm{~h}$ of CAF treatment, $\mathrm{n}=3$, normalized 
to the Rplp0 housekeeping gene, +/- SEM, ${ }^{*} \mathrm{p}$-val $\leq 0.05,{ }^{* *} \mathrm{p}$-val $\leq 0.01,{ }^{* * *} \mathrm{p}$-val $\leq 0.005$. B: Immunofluorescence staining of Ezh2 and Suz12 in CAF-treated rG-2 cells, corresponding to Fig. I 2G.

A.
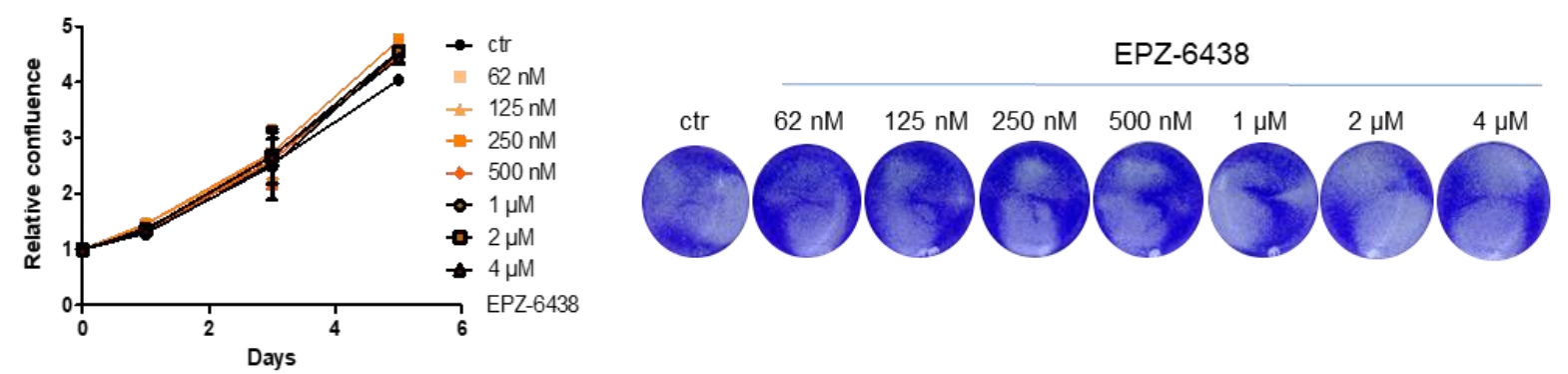

B.
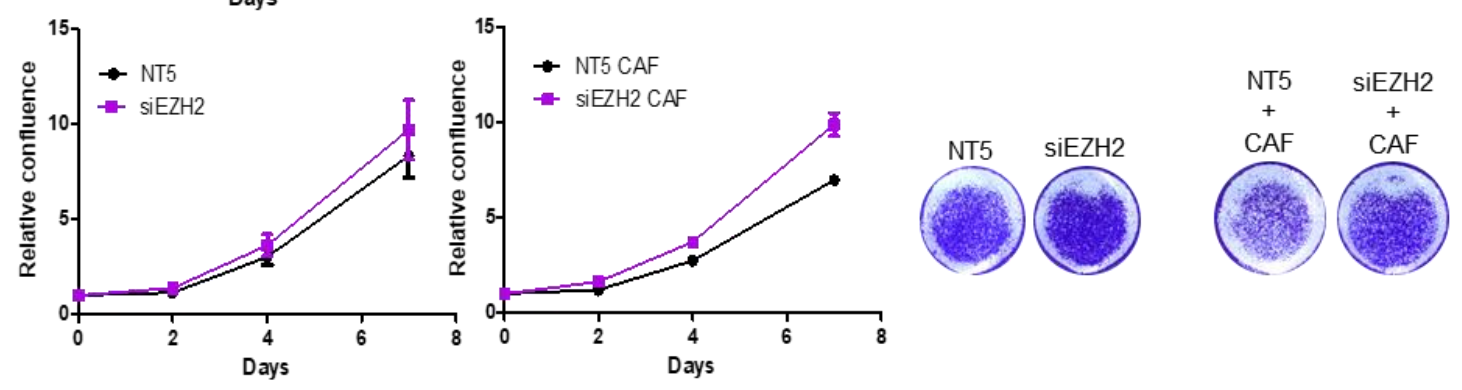

Fig. I S3 EZH2 inhibition and knockdown in MDA-MB-468 cells. A: Proliferation assay of EPZ-6438treated MDA-MD-468 cells using Celigo (left) and crystal violet staining at day 7 (right). B: Proliferation measurement (left) and crystal violet staining (right) of cells upon EZH2 knockdown with or without 1/32 CAF-chemotherapy treatment.

HCT116

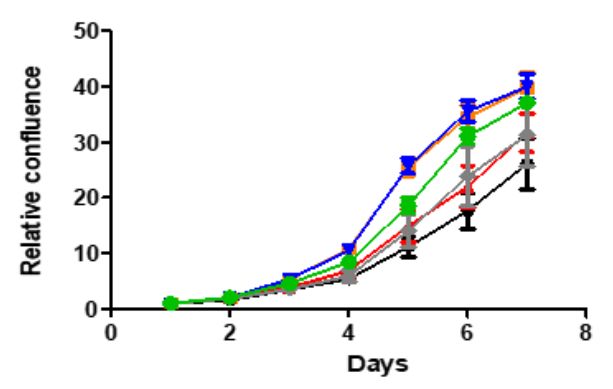

TFK1

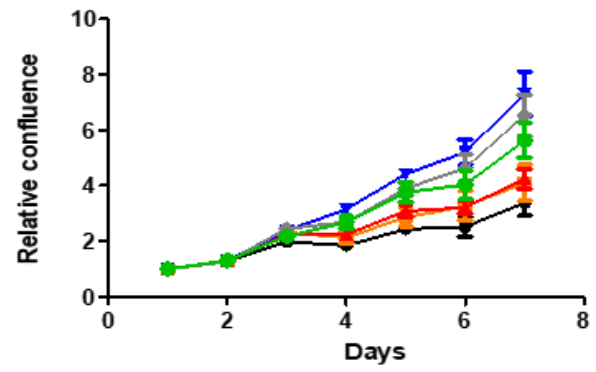

HT29

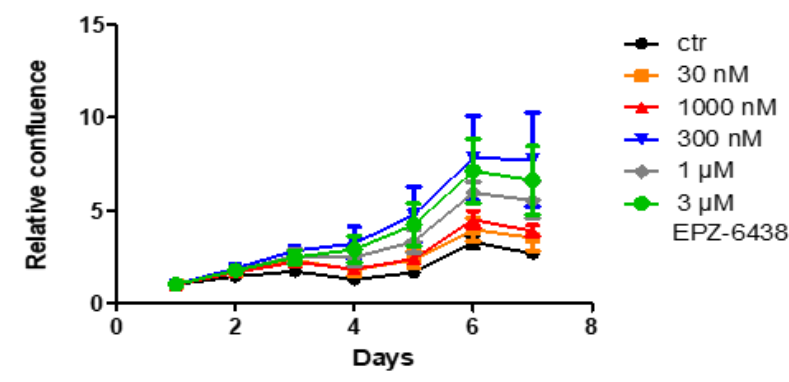

EGI-1

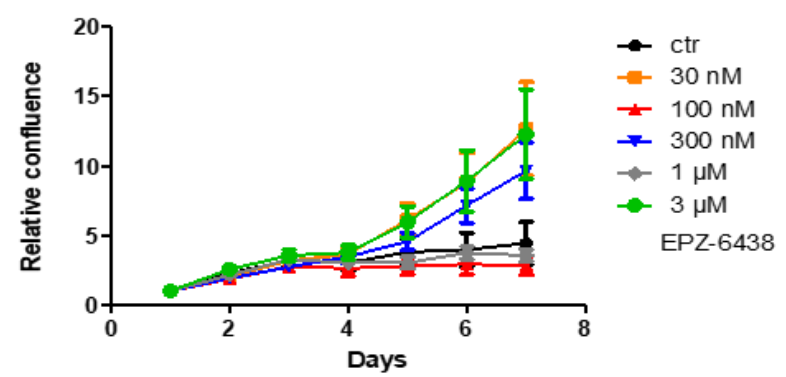

Fig. I S4 EZH2 inhibition in human colorectal and bile duct carcinoma cell lines. Proliferation assay of HCT116 and HT29 (colorectal cancer), TFK1 and EGI-1 (bile duct carcinoma) cells treated with increasing EPZ-6438 concentrations using Celigo $(n=3)$. 
wo CAF

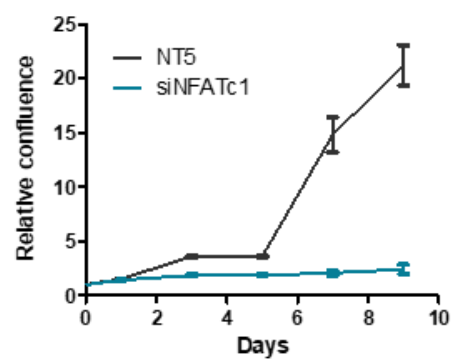

$1 / 32 \mathrm{CAF}$

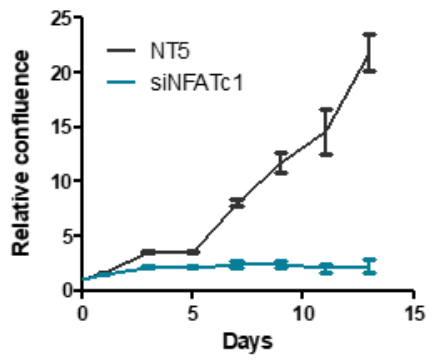

sINFATc1

Fig. I S5 NFATc1 knockdown in MDA-MB-468 cells treated with and without 1/32 CAFchemotherapy. Proliferation assay of EPZ-6438-treated MDA-MD-468 cells using Celigo (left) and crystal violet staining at day 7 (right).
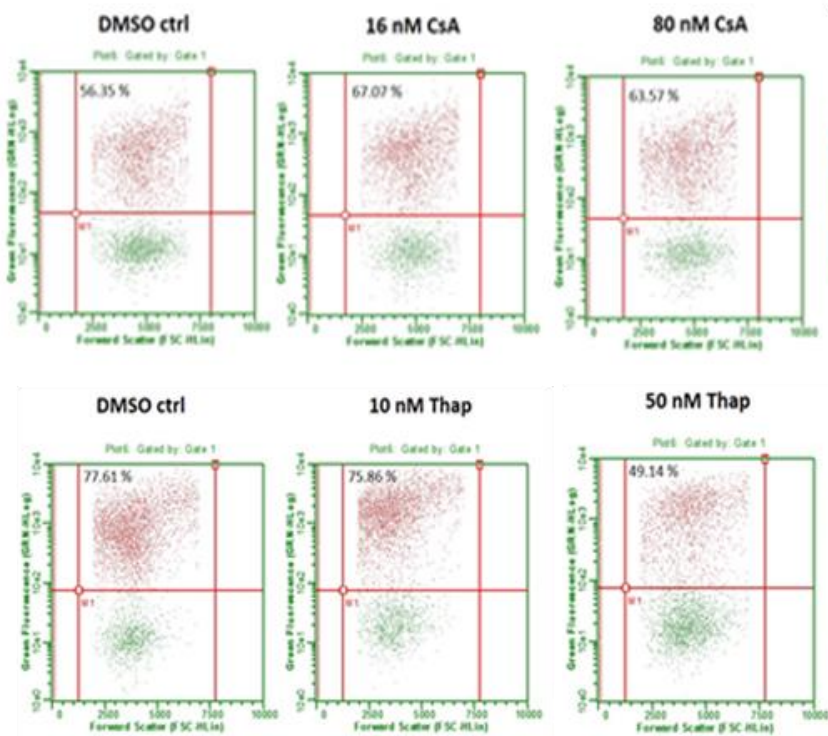
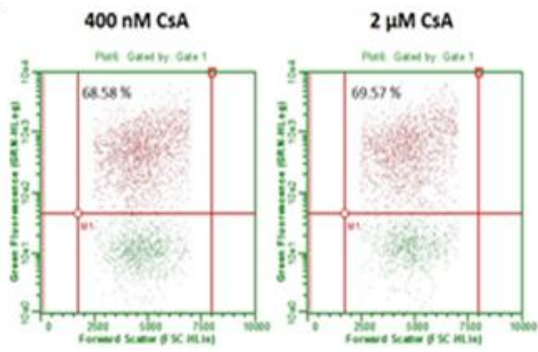

Fig. I S6: Inhibition and activation of NFATc1 leads to epithelial-mesenchymal phenotype changes in pG-2 cells. pG-2 cells were treated for $48 \mathrm{~h}$ with different concentrations of cyclosporine $A$ (CsA) or thapsigargin (Thap), harvested and stained with the conjugated antibody FITC-EpCAM, and analyzed by flow cytometry.

Table I S1. Epigenetic regulators list

\begin{tabular}{|l|l|l|l|l|l|l|l|l|}
\hline AIRE & CBX2 & DPF2 & JARID2 & L3MBTL3 & NSD1 & $\begin{array}{l}\text { PRDM } \\
11\end{array}$ & SETD1A & SMYD5 \\
\hline AOF2 & CBX3 & DPF3 & $\begin{array}{l}\text { JHDM1 } \\
\text { D }\end{array}$ & L3MBTL4 & OGT & $\begin{array}{l}\text { PRDM } \\
12\end{array}$ & SETD1B & SP100 \\
\hline ARID1A & CBX4 & DPY30 & $\begin{array}{l}\text { JMJD1 } \\
\text { C }\end{array}$ & LEO1 & PADI2 & $\begin{array}{l}\text { PRDM } \\
13\end{array}$ & SETD2 & SP110 \\
\hline ARID4A & CBX5 & DZIP3 & $\begin{array}{l}\text { JMJD2 } \\
\text { A }\end{array}$ & LRWD1 & PADI4 & $\begin{array}{l}\text { PRDM } \\
14\end{array}$ & SETD3 & SP140 \\
\hline ARID4B & CBX6 & EED & JMJD4 & MBD1 & PAF1 & $\begin{array}{l}\text { PRDM } \\
15\end{array}$ & SETD4 & SP140L \\
\hline ASF1A & CBX7 & EHMT1 & JMJD6 & MBD2 & PBRM1 & $\begin{array}{l}\text { PRDM } \\
16\end{array}$ & SETD5 & SRCAP \\
\hline ASF1B & CBX8 & EHMT2 & JMJD7 & MBD3 & PCAF & $\begin{array}{l}\text { PRDM } \\
2\end{array}$ & SETD6 & SSRP1 \\
\hline
\end{tabular}




\begin{tabular}{|c|c|c|c|c|c|c|c|c|}
\hline ASH1L & $\begin{array}{l}\text { CCDC1 } \\
01\end{array}$ & ELP3 & JMJD8 & MBD4 & PCGF1 & $\begin{array}{l}\text { PRDM } \\
4\end{array}$ & SETD7 & $\begin{array}{l}\text { SUPT16 } \\
\mathrm{H}\end{array}$ \\
\hline ASH2L & CDC73 & EP300 & KAT2A & MBD5 & PCGF2 & $\begin{array}{l}\text { PRDM } \\
5\end{array}$ & SETD8 & SUPT6H \\
\hline ASXL1 & CDY1 & EP400 & KAT2B & MBD6 & PCGF3 & $\begin{array}{l}\text { PRDM } \\
6\end{array}$ & SETD9 & SUPT7L \\
\hline ASXL2 & CDY1B & EPC1 & KAT5 & MBTD1 & PCGF5 & $\begin{array}{l}\text { PRDM } \\
7\end{array}$ & SETDB1 & $\begin{array}{l}\text { SUV39H } \\
1\end{array}$ \\
\hline ASXL3 & CDY2A & EPC2 & KAT6A & MECP2 & PCGF6 & $\begin{array}{l}\text { PRDM } \\
8\end{array}$ & SETDB2 & $\begin{array}{l}\text { SUV39H } \\
2\end{array}$ \\
\hline ATAD2 & CDY2B & EZH1 & KAT6B & MEN1 & PHC1 & $\begin{array}{l}\text { PRDM } \\
9\end{array}$ & SETMAR & $\begin{array}{l}\text { SUV420 } \\
\mathrm{H} 1\end{array}$ \\
\hline ATAD2B & CDYL & $\mathrm{EZH} 2$ & KAT7 & MGMT & PHC2 & PRMT1 & SFMBT1 & $\begin{array}{l}\text { SUV420 } \\
\mathrm{H} 2\end{array}$ \\
\hline ATRX & CDYL2 & FANCL & KAT8 & MINA & PHC3 & $\begin{array}{l}\text { PRMT1 } \\
0\end{array}$ & SFMBT2 & SUZ12 \\
\hline ATXN7 & CECR2 & FBX010 & KDM1A & MLLT10 & PHF1 & PRMT2 & SHPRH & TADA3L \\
\hline $\begin{array}{l}\text { ATXN7L } \\
3\end{array}$ & $\begin{array}{l}\text { CHAF1 } \\
\text { A }\end{array}$ & FBX011 & KDM1B & MLLT6 & PHF10 & PRMT3 & SIN3A & TAF1 \\
\hline BAZ1A & $\begin{array}{l}\text { CHAF1 } \\
\text { B }\end{array}$ & G2E3 & KDM2A & MORC3 & PHF11 & PRMT5 & SIN3B & TAF1L \\
\hline BAZ1B & CHD1 & $\begin{array}{l}\text { GADD4 } \\
5 A\end{array}$ & KDM2B & MORC4 & PHF12 & PRMT6 & SIRT1 & TAF3 \\
\hline BAZ2A & CHD1L & HAT1 & KDM3A & MORF4L1 & PHF13 & PRMT7 & SIRT2 & TAF8 \\
\hline BAZ2B & CHD2 & HDAC1 & KDM3B & MORF4L2 & PHF14 & PRMT8 & SIRT6 & TDRD3 \\
\hline BMI1 & CHD3 & $\begin{array}{l}\text { HDAC1 } \\
0\end{array}$ & KDM4A & $\begin{array}{l}\text { MPHOSP } \\
\mathrm{H} 8\end{array}$ & PHF15 & $\begin{array}{l}\text { PROM } \\
1\end{array}$ & SIRT7 & TET1 \\
\hline BPTF & CHD4 & $\begin{array}{l}\text { HDAC1 } \\
1\end{array}$ & KDM4B & MSL3L1 & PHF16 & $\begin{array}{l}\text { PYGO } \\
1\end{array}$ & SKB1 & TET2 \\
\hline BRD1 & CHD5 & HDAC2 & KDM4C & MTA1 & PHF17 & $\begin{array}{l}\text { PYGO } \\
2\end{array}$ & $\begin{array}{l}\text { SMARCA } \\
1\end{array}$ & TET3 \\
\hline BRD2 & CHD6 & HDAC3 & KDM4D & MTA2 & PHF19 & RAG2 & $\begin{array}{l}\text { SMARCA } \\
2\end{array}$ & TRIM24 \\
\hline BRD3 & CHD7 & HDAC4 & KDM4E & MTF2 & PHF2 & RBBP4 & $\begin{array}{l}\text { SMARCA } \\
4\end{array}$ & TRIM28 \\
\hline BRD4 & CHD8 & HDAC5 & KDM5A & MYSM1 & PHF20 & RBBP7 & $\begin{array}{l}\text { SMARCA } \\
5\end{array}$ & TRIM33 \\
\hline BRD7 & CHD9 & HDAC6 & KDM5B & MYST2 & $\begin{array}{l}\text { PHF20 } \\
\text { L1 }\end{array}$ & RING1 & $\begin{array}{l}\text { SMARCA } \\
\text { D1 }\end{array}$ & TRIM66 \\
\hline BRD8 & $\begin{array}{l}\text { CREBB } \\
\mathrm{P} \\
\end{array}$ & $\begin{array}{l}\text { HDAC7 } \\
\mathrm{A}\end{array}$ & KDM5C & MYST3 & $\begin{array}{l}\text { PHF21 } \\
\text { A }\end{array}$ & $\begin{array}{l}\text { RNF16 } \\
8 \\
\end{array}$ & $\begin{array}{l}\text { SMARCA } \\
\text { L1 }\end{array}$ & TRRAP \\
\hline BRD9 & CTR9 & HDAC8 & KDM5D & MYST4 & $\begin{array}{l}\text { PHF21 } \\
\text { B }\end{array}$ & RNF2 & $\begin{array}{l}\text { SMARCB } \\
1\end{array}$ & UHRF1 \\
\hline BRDT & CXXC1 & HDAC9 & KDM6A & NAP1L1 & PHF23 & RNF20 & $\begin{array}{l}\text { SMARCC } \\
1\end{array}$ & UHRF2 \\
\hline BRMS1 & DIDO1 & HIRA & KDM6B & NAP1L2 & PHF3 & RNF40 & $\begin{array}{l}\text { SMARCC } \\
2\end{array}$ & USP22 \\
\hline $\begin{array}{l}\text { BRMS1 } \\
\mathrm{L}\end{array}$ & DMAP1 & HLTF & KDM8 & NAP1L3 & PHF5A & RNF8 & $\begin{array}{l}\text { SMARCD } \\
1\end{array}$ & UTY \\
\hline BRPF1 & $\begin{array}{l}\text { DNAPT } \\
\text { P3 }\end{array}$ & HTATIP & KMT2A & NAP1L4 & PHF6 & RSF1 & $\begin{array}{l}\text { SMARCD } \\
2\end{array}$ & WDR82 \\
\hline BRPF3 & DNMT1 & ING1 & KMT2B & NAP1L5 & PHF7 & $\begin{array}{l}\text { RUVBL } \\
1\end{array}$ & $\begin{array}{l}\text { SMARCD } \\
3\end{array}$ & WDR5 \\
\hline
\end{tabular}




\begin{tabular}{|l|l|l|l|l|l|l|l|l|}
\hline BRWD1 & $\begin{array}{l}\text { DNMT3 } \\
\text { A }\end{array}$ & ING2 & KMT2C & NAP1L6 & PHF8 & $\begin{array}{l}\text { RUVBL } \\
2\end{array}$ & $\begin{array}{l}\text { SMARCE } \\
1\end{array}$ & WHSC1 \\
\hline BRWD3 & $\begin{array}{l}\text { DNMT3 } \\
\text { B }\end{array}$ & ING3 & KMT2D & NAT10 & PHIP & RYBP & SMYD1 & $\begin{array}{l}\text { WHSC1L } \\
1\end{array}$ \\
\hline $\begin{array}{l}\text { C140rf1 } \\
69\end{array}$ & $\begin{array}{l}\text { DNMT3 } \\
\text { L }\end{array}$ & ING4 & KMT2E & NCOA1 & PHRF1 & $\begin{array}{l}\text { SCMH } \\
1\end{array}$ & SMYD2 & $\begin{array}{l}\text { ZCWPW } \\
1\end{array}$ \\
\hline CARM1 & DOT1L & ING5 & $\begin{array}{l}\text { L3MBT } \\
\text { L1 }\end{array}$ & NCOA2 & PRDM1 & SCML2 & SMYD3 & $\begin{array}{l}\text { ZCWPW } \\
2\end{array}$ \\
\hline CBX1 & DPF1 & INTS12 & $\begin{array}{l}\text { L3MBT } \\
\text { L2 }\end{array}$ & NCOA3 & $\begin{array}{l}\text { PRDM1 } \\
0\end{array}$ & SET & SMYD4 & ZMYN11 \\
\hline & & & & & & & & ZMYND8 \\
\hline
\end{tabular}

\section{Funding}

Erich und Gertrud Roggenbuck-Stiftung zur Förderung der Krebsforschung.

\section{Acknowledgements}

The authors would like to thank the group of Prof. Johnsen for constructive discussions and help.

\section{Contributor Information}

Iga K. Mieczkowska, iga.dudzicz@med.uni-goettingen.de

Geske E. Schmidt, geske.schmidt@med.uni-goettingen.de

Lukas C. Müller-Kirschbaum, I.muellerkirschbau@stud.uni-goettingen.de

Garyfallia Pantelaiou,gar_pantelaiou@yahoo.gr

Evangelos Prokakis, eprokak@gwdg.de

Taras Velychko, taras.velychko@gmail.com

Frauke Alves, falves@gwdg.de

Madhobi Sen, madhobi.sen@zentr.uni-goettingen.de

Florian Wegwitz, fwegwit@gwdg.de 


\subsection{Chapter II Report}

\section{Therapeutical potential of HDACs in chemotherapy resistance in triple-negative breast cancer}

\section{Iga K. Mieczkowska ${ }^{1}$, Julio Abril Garrido ${ }^{1}$, Florian Wegwitz ${ }^{1}$}

1 Department of General, Visceral and Pediatric Surgery, University Medical Center Göttingen, Göttingen Center for Molecular Biosciences, Göttingen, Germany iga.dudzicz@med.uni-goettingen.de; julio.abrilgarrido@stud.uni-goettingen.de; fwegwit@med.uni-goettingen.de

\section{Introduction}

The most commonly diagnosed cancers in women are malignancies of the breast (World Health Organisation, 2018). Triple-negative breast cancer (TNBC) is the most aggressive and heterogeneous breast cancer subtype (Perou et al., 2000). Due to lack of estrogen receptor (ER) progesterone receptor (PR) and human epidermal growth factor 2 (HER2) expression, chemotherapy treatment is one of the most common therapy in TNBC. Conventional chemotherapy including platinum agents, such as carboplatin and cisplatin, paclitaxel or 5-fluorouracil before or after surgical resection remain the standard approach in the clinic (Isakoff, 2010; Sirohi et al., 2008).

Unfortunately, cancer relapse occurs quite frequently among TNBC patients followed by chemotherapy (O'Reilly et al., 2015). Epigenetic changes are involved in chemotherapy resistance as many studies and our previous data report (Mieczkowska et al., 2019, unpublished, J. Han et al., 2019; Pineda et al., 2019). Histone deacetylases (HDACs) are a class of epigenetic regulators very frequently altered in cancers including breast cancer (Krusche et al., 2005; K. M. Sakamoto \& Aldana-Masangkay, 2011; Shan et al., 2017). HDACs modulate the gene expression by among other controlling the deacetylation/acetylation balance at lysine residues of histones, together 
with histone acetyltransferases (HAT), (Grunstein, 1997). The deacetylation of histones leads to chromatin condensation resulting in repression of transcription (Roth et al., 2001). We can distinguish four classes of HDAC family: class I (HDAC1, $-2,-3$ and -8 ), class Ila (4, -5, -7 and -9), IIb (HDAC6 and -10), class III (sirtuins) and class IV (HDAC11) (Gregoretti et al., 2004; Seto \& Yoshida, 2014). HDAC-triggered abnormality in pivotal gene expression can lead to tumor invasiveness (Richon et al., 2000). As a consequence, anti-cancer potential of therapeutic strategies targeting HDACs have been several times demonstrated in vitro and in vivo, therefore being very attractive for the development of novel drugs (Bolden et al., 2006). The best studied and FDAapproved pan-HDAC inhibitors are Vorinostat (SAHA) and Panobinostat ( $\mathrm{LBH}-589$ ), both strongly targeting HDACs class I and II (Duvic et al., 2007; San-Miguel et al., 2013; Stahl et al., 2016). HDACs inhibitors are clinically used for cutaneous T-cell lymphoma (CTCLs) (Lopez et al., 2018). Using HDACs inhibitors improved patient outomes with myeloma. However, beneficial therapeutical effects were not obtained in solid tumors (Bolden et al., 2006).

In TNBC, SAHA was reported as the most promising therapeutic treatment, however its efficiency is limited as a single drug (Garmpis et al., 2017). In the present project, we identified an upregulation of Hdac4, Hdac7 and Hdac8 in murine WAP-T TNBC cells surviving a cytotoxic therapy. We therefore aimed to study the potential of treatments specifically targeting one of these HDACs and to determine possible benefits of a combination with chemotherapy in TNBC.

\section{Results}

\section{CAF chemotherapy-induced EMT changes in TNBC}

We previously showed that WAP-T cells (pG-2) surviving a CAF (Cyclophosphamide, Doxorubicin/Adriamicin, and 5-Fluorouracil/5-FU) chemotherapy treatment adopted a more mesenchymal phenotype, pointing at an involvement of epithelial-to-mesenchymal transition program in the chemotherapy resistance (Mieczkowska et al., 2019, manuscript under submission). To determine if human TNBC cells undergo similar transcriptional program changes upon survival to cytotoxic 
therapies, HCC1806 cells where treated with increasing concentrations of a CAFchemotherapy as well as cisplatin and paclitaxel. Concentration of $2.5 \mu \mathrm{M}$ cisplatin, 2.5 $\mathrm{nM}$ paclitaxel and 1/16 CAF (30 ng/ml Cyclophosphamide, $0.62 \mu \mathrm{g} / \mathrm{ml}$ Doxorubicin, $0.62 \mu \mathrm{g} / \mathrm{ml} 5-\mathrm{FU}$ ) showed the optimal results for our purpose (Fig II S1), killing the vast majority of the cells, while allowing the regrowth of more resistant cell phenotypes (Fig II 1A). Strikingly, gene expression analyses of treated HCC1806 cells identified an increased expression of mesenchymal markers (SNA11, N-CADHERIN, VIMENTIM) in cells surviving the different treatments, pointing at the occurrence of an EMT also in the human context (Fig. II 1B). 
A
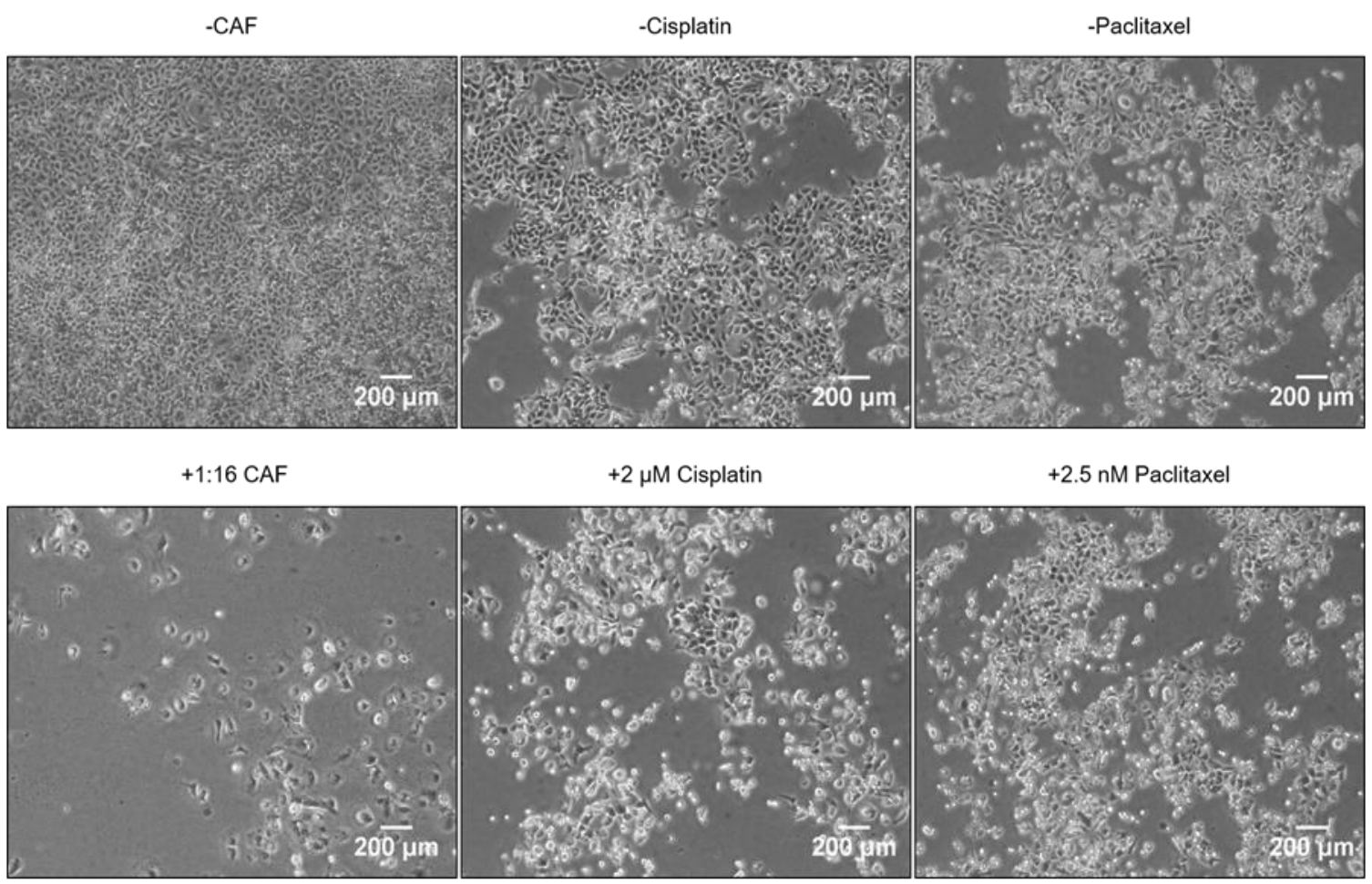

B
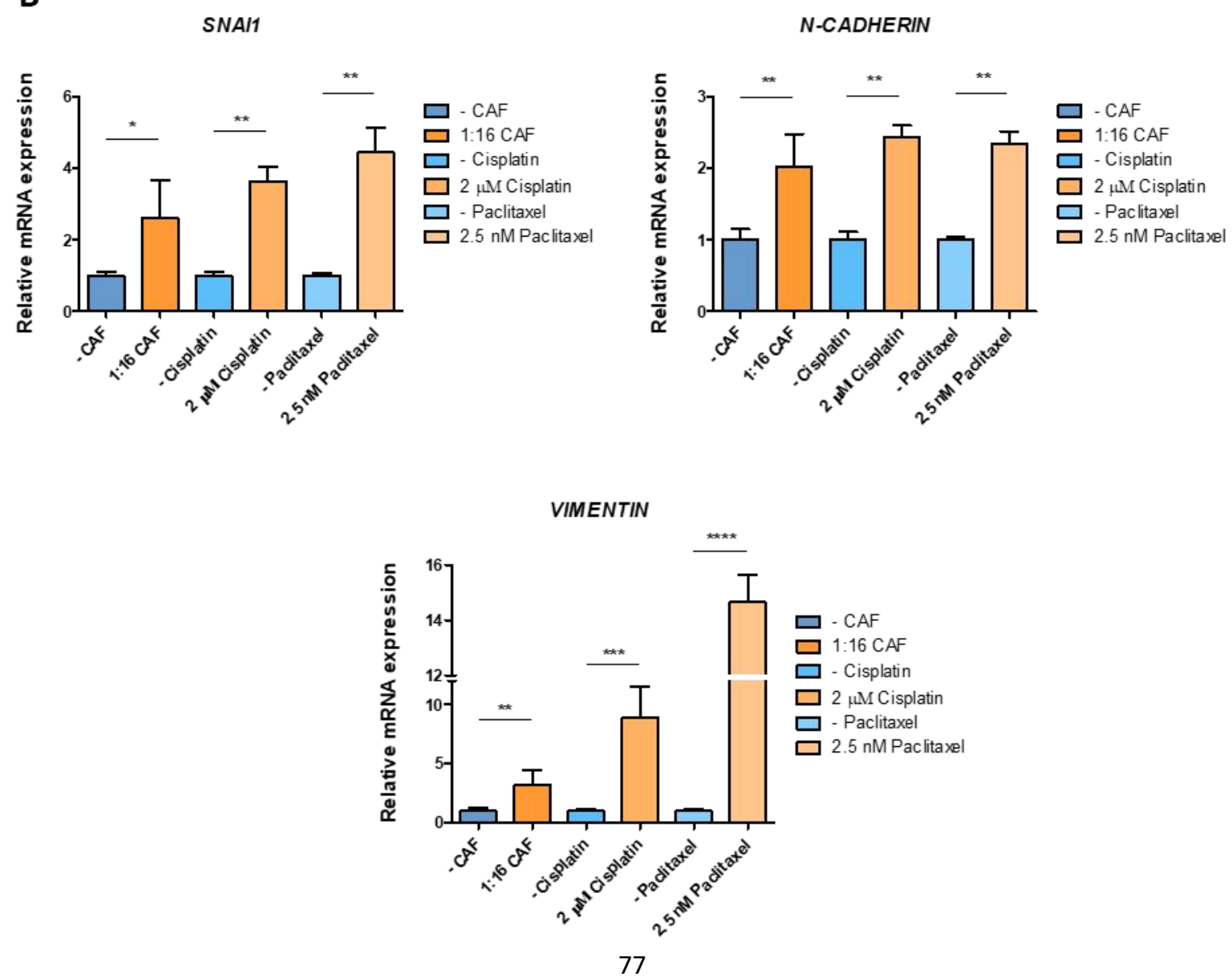
Figure II 1. A: Human TNBC cells undergo EMT upon survival to different cytotoxic treatments. $24 \mathrm{hr}$ after seeding, the cells were treated with either $1 / 16 \mathrm{CAF}(30 \mathrm{ng} / \mathrm{ml}$ Cyclophosphamide, $0.62 \mu \mathrm{g} / \mathrm{ml}$ Doxorubicin, $0.62 \mu \mathrm{g} / \mathrm{ml} 5-\mathrm{FU}), 2 \mu \mathrm{M}$ cisplatin or $2.5 \mathrm{nM}$ paclitaxel for 48 hours. A: bright field pictures of HCC1806 upon various chemotherapy treatments. Microscope: Nikon ECLIPSE TS100-F with 4x objective. B: Gene expression analysis of EMT markers in HCC1806 cell line upon treatment, assessed by qRT-PCR. mRNA expression values were normalized to the housekeeping gene expression Rp/p0 and calibrated to the respective untreated controls. Average mRNA expression values are given \pm standard deviation (SD). Experiments were conducted in biological duplicates $(n=2)$ with technical triplicates. ${ }^{*}: p<0.05 ;{ }^{* *}: p<0.01$.

In our previous work, mRNA-sequencing (mRNA-seq) analyses were performed to study the mechanisms involved in WAP-T cell survival to chemotherapy (Mieczkowska et al., 2019, manuscript under submission). We thereby identified the enrichment of EMT and cancer stem cell (CSC) signatures signature accompanying more resistant WAP-T cancer cell phenotypes. Interestingly, signatures pointing at epigenetic dysregulations were enriched. As epigenetic regulatory pathway are known to control EMT- and CSCs - transcriptional programs (Lu \& Kang, 2019) we focused on the regulation of epigenetic factors upon CAF treatment. Here, we observed that the majority of these factors were down-regulated $(n=64)$ and that only a few were upregulated ( $\mathrm{n}=16$ ). We notably observed that Hdac4, Hdac7 (class Ila) and Hdac8 (class I) figured among the upregulated genes. Hdac4, -7 and -8 overexpression was reported to be associated with poor survival and tumor invasiveness (Hsieh et al., 2016; Zeng et al., 2016; Zhu et al., 2011). We performed qRT-PCRs on pG-2 and rG-2 cells, a chemoresistant resistant variant of the parental pG-2 cell line (Mieczkowska et al., 2019, manuscript under submission), to validate our findings. Indeed, CAF treatment of pG-2 cells increased mRNA expression levels of Hdac4, and its levels were maintained high in the resistant cells, independent of treatment. Hdac7 was almost three times upregulated in treated parental cells. Interestingly, rG-2 cells displayed sensibly the same high levels of Hdac7 expression as treated pG-2 cells and its expression even stronger upon cytotoxic treatment. Finally, levels of $\mathrm{Hdac} 8$ were only moderately upregulated upon CAF treatment of parental cells and its levels were only significantly higher in the treated resistant cells. Taken together, we confirmed the upregulation of Hdac4, 7 and 8 upon cytotoxic treatment and observed that high levels of these genes are associated with resistant phenotypes. We therefore asked if 
interfering with these factors via siRNA mediated silencing would impact the growth and resistance properties of these cells. We therefore performed crystal violet staining and found that loss of Hdac4, -7 and -8 slightly reduced the proliferation of pG-2 cells and only marginally the proliferation of rG-2 cells, when cultured under normal conditions. Strikingly, the proliferation of $\mathrm{pG}-2$ and $\mathrm{rG}-2$ was much stronger affected by siRNA treatment when co-treated with low doses of CAF (Fig. II 2C), pointing at a sensitization of the cells to the chemotherapy. 
A

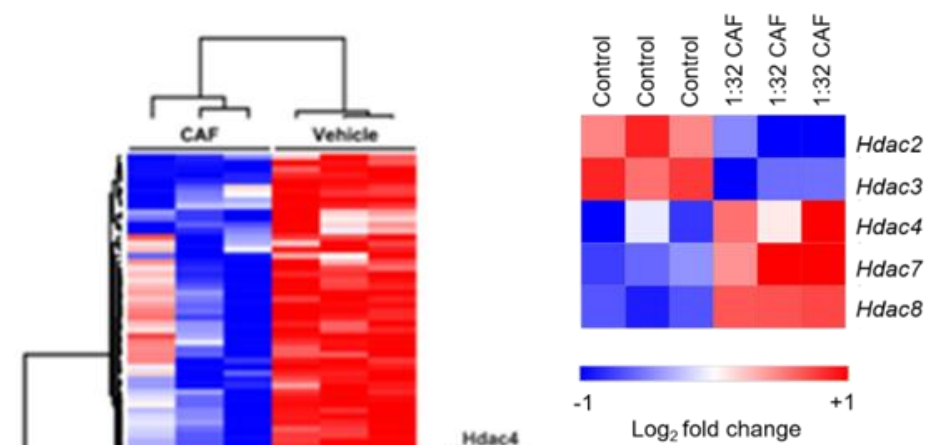

B
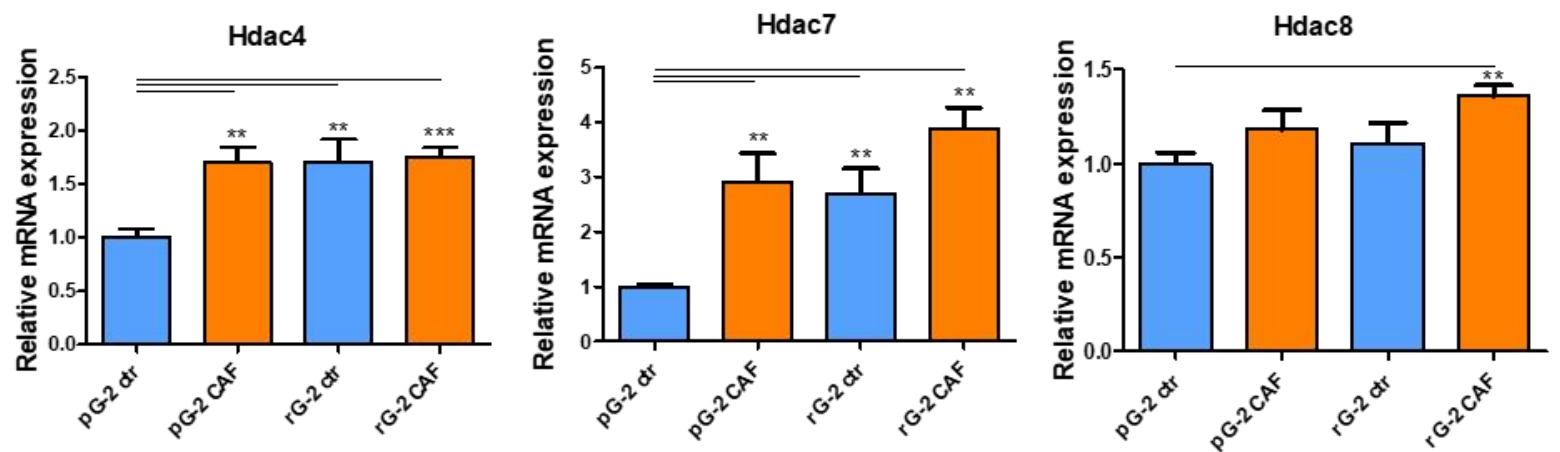

C

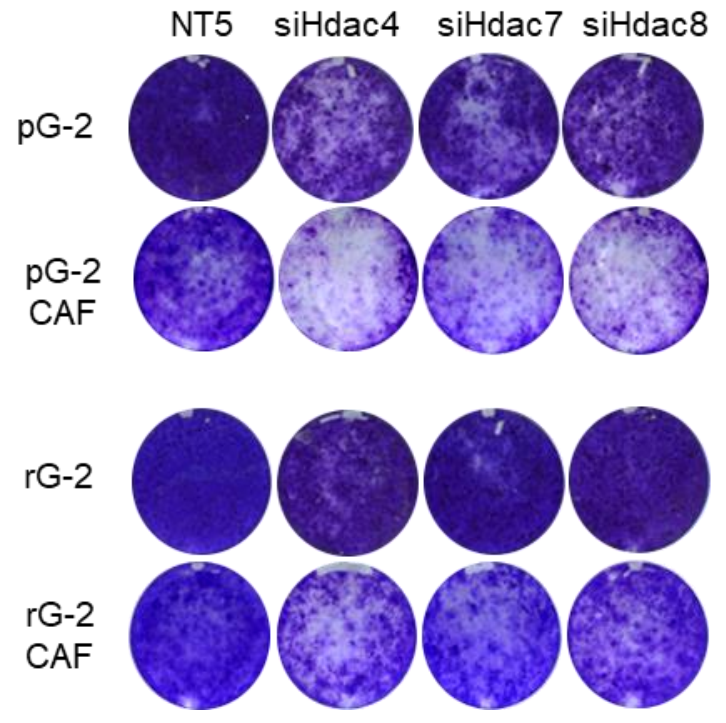

Figure II 2. Upregulation of Hdac4, -7 and -8 upon CAF-chemotherapy treatment in G-2 cells is necessary for their survival. A: Heatmaps showing epigenetic factors (left panel) and several Hdac 
genes (right panel) significantly regulated upon 48 hours CAF treatment $(312,5 \mathrm{ng} / \mathrm{ml}$ cyclophosphamide, $15,6 \mathrm{ng} / \mathrm{ml}$ doxorubicin and 312,5 $\mathrm{ng} / \mathrm{ml} 5-\mathrm{FU}$ ) in pG-2 cells ( $p$ adjusted value $<0.05, \mathrm{n}=3$ biological replicates). Expression values are displayed as Z-score. B: Validation of Hdac4, Hdac7 and Hdac8 gene regulation in pG-2 and rG-2 cells (ctr) upon 48 hours CAF. mRNA expression values were normalized to the housekeeping gene RplpO and to the respective untreated controls. Average mRNA expression values are given \pm standard deviation (SD). Experiments were conducted in biological duplicates $(n=3)$. T-test: ${ }^{*} p<0.05 ;{ }^{* *} p<0.01 ;{ }^{* * *} p<0.001$. C: Proliferation assay using crystal violet staining upon Hdac4, Hdac7 and Hdac8 knockdown in pG-2 and rG-2 cell lines with or without CAF treatment (CAF), $\mathrm{n}=2$ biological replicates.

\section{EMT modulation and TNBC cell survival upon HDAC4, -7 and -8 loss}

To extend our results to the human situation, we examined the influence of HDAC4, -7 and -8 loss in human HCC1806 cells. Decreased cell growth rate under normal conditions was observed upon HDAC4, -7 and -8 knockdown (Fig. II 3A). Interestingly, in a similar manner as for murine cells, we observed a clear sensitization of the human TNBC cells to low concentration of CAF treatment (1/256) when combined with HDAC4, -7 and -8 knockdown (Fig. II 3B). We next asked whether depletion of HDAC4, 7 or 8 in human cells could also result in an impairment of the EMT transcriptional program. Surprisingly, we observed that solely HDAC8 loss lead to the downregulation of the mesenchymal markers (SNAl1, ZEB1, SLUG) (Fig. II 3C). 
A

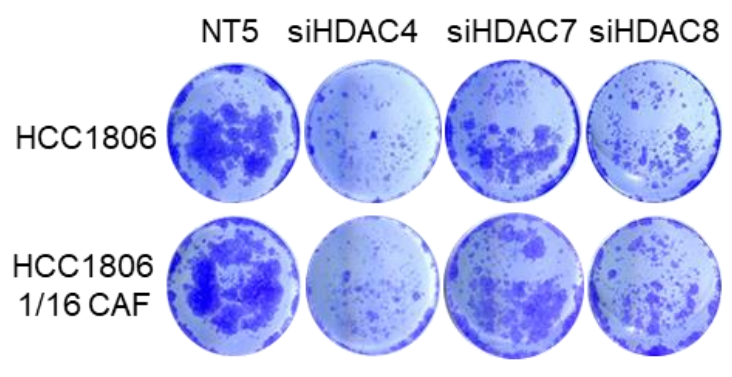

C

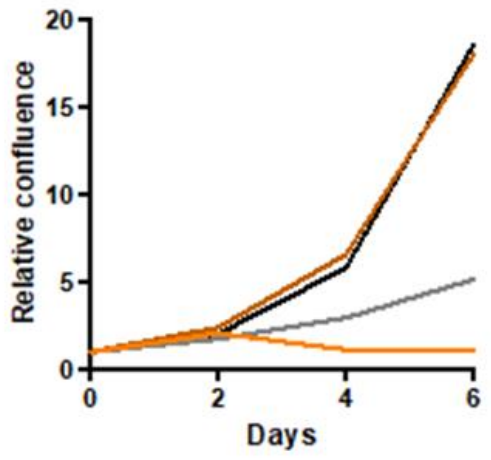

- NT5

- 1/256 CAF

- SiHDAC4

- siHDAC4 1/256 CAF
B

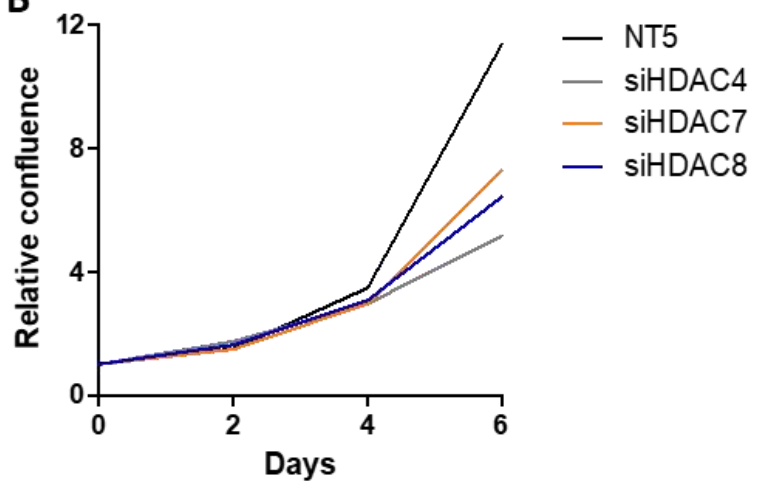

D
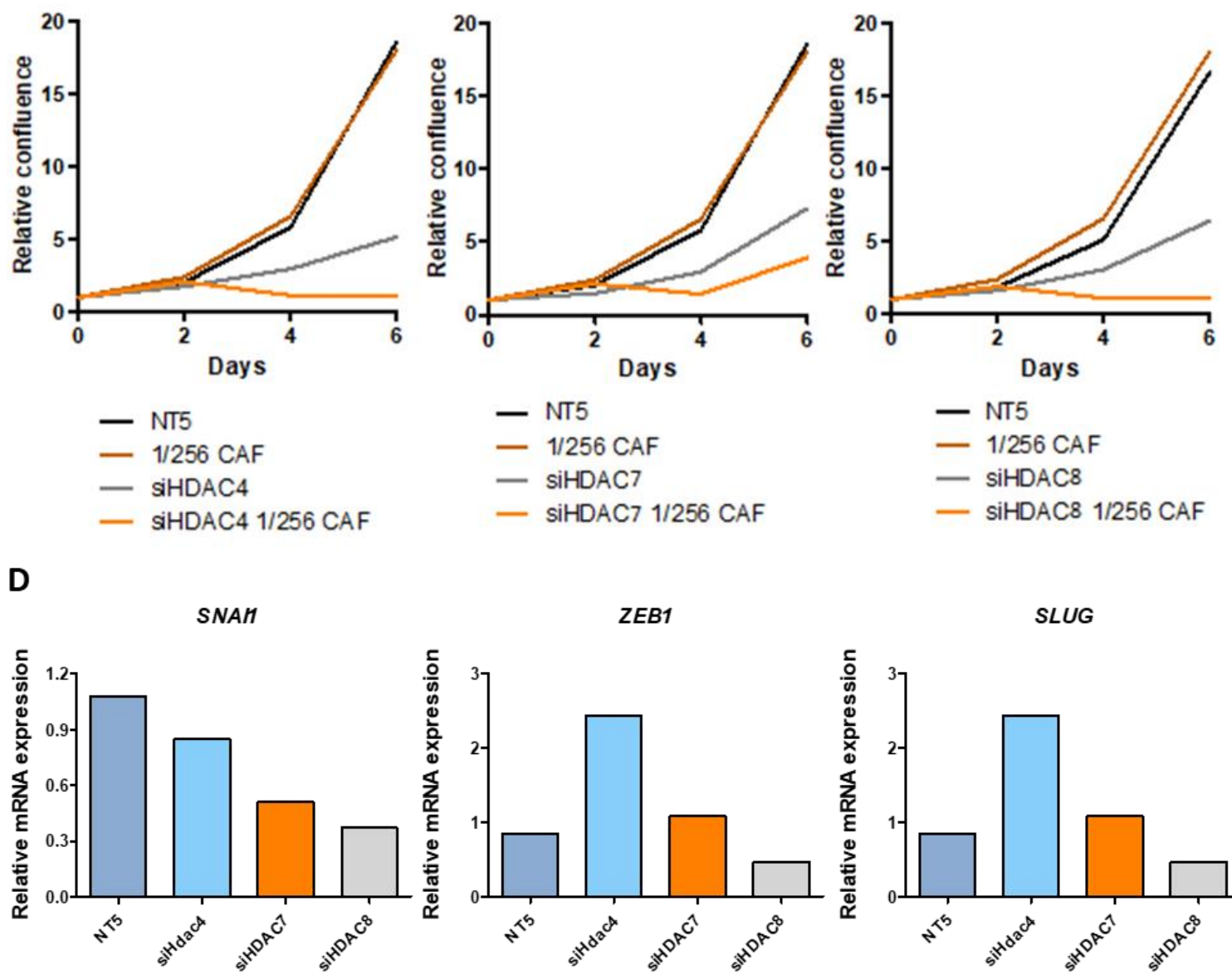

- NT5

- 1/256 CAF

- SiHDAC8

- siHDAC8 1/256 CAF

ZEB1

SLUG
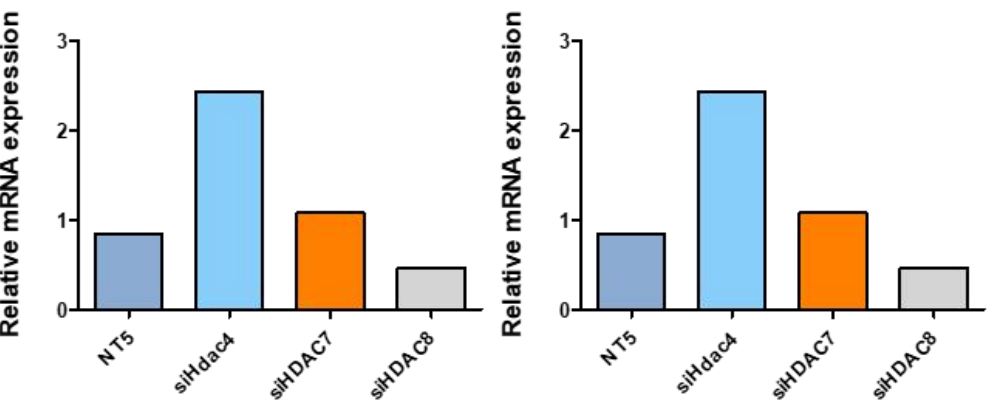

Figure II 3. Impact of HDAC4, -7 and -8 loss in HCC1806 cells. A and B: Proliferation assays of HCC1806 cells upon HDAC4, -7 and -8 knockdown with or without CAF-treatment $(30 \mathrm{ng} / \mathrm{ml}$ Cyclophosphamide, $0.62 \mu \mathrm{g} / \mathrm{ml}$ Doxorubicin, $0.62 \mu \mathrm{g} / \mathrm{ml} 5-\mathrm{FU}$ ), assessed via crystal violet staining (A) and Celigo cell cytometer measurement. C: Proliferation assays of HCC1806 cells upon HDAC4, -7 and -8 knockdown with or without low dose of CAF-treatment (1/256), assessed via Celigo D: Gene expression analysis of EMT markers in HCC1806 cell line using qRT-PCR. mRNA expression values were normalized to $R p / p 0$ and to the respective untreated controls. Average mRNA expression values are given \pm standard deviation (SD), $\mathrm{n}=1$, technical triplicates. 
Following, we examined the potential of a co-treatment with chemotherapy and HDAC inhibitors in TNBC. Although silencing of HDAC4 and HDAC7 sensitized pG-2 and HCC1806 cells to cytotoxic therapies, the well-established HDAC class II inhibitors TMP269 and TMP195 inhibiting both HDAC4 and -7 could not impair cell viability when combined with CAF, cisplatin or paclitaxel (data not shown). As only HDAC8 loss reduced EMT induction in HCC1806, we decided to investigate the clinical potential of HDAC8 inhibition using PCl-34051 in combination with cytotoxic drugs in pG-2 (Fig. II 4 A) and rG-2 cells (Fig. II 4B). In pG-2 cells, we could observe a sensitization to the therapy when PCl-34051 and CAF-chemotherapy were combined. Moreover, HDAC8 inhibition alone and in combination with CAF lead to significant cell growth impairment in the resistant rG-2 cells. Together, our findings identified HDAC8 as an attractive target to increase efficiency of cytotoxic therapies in TNBC.

A
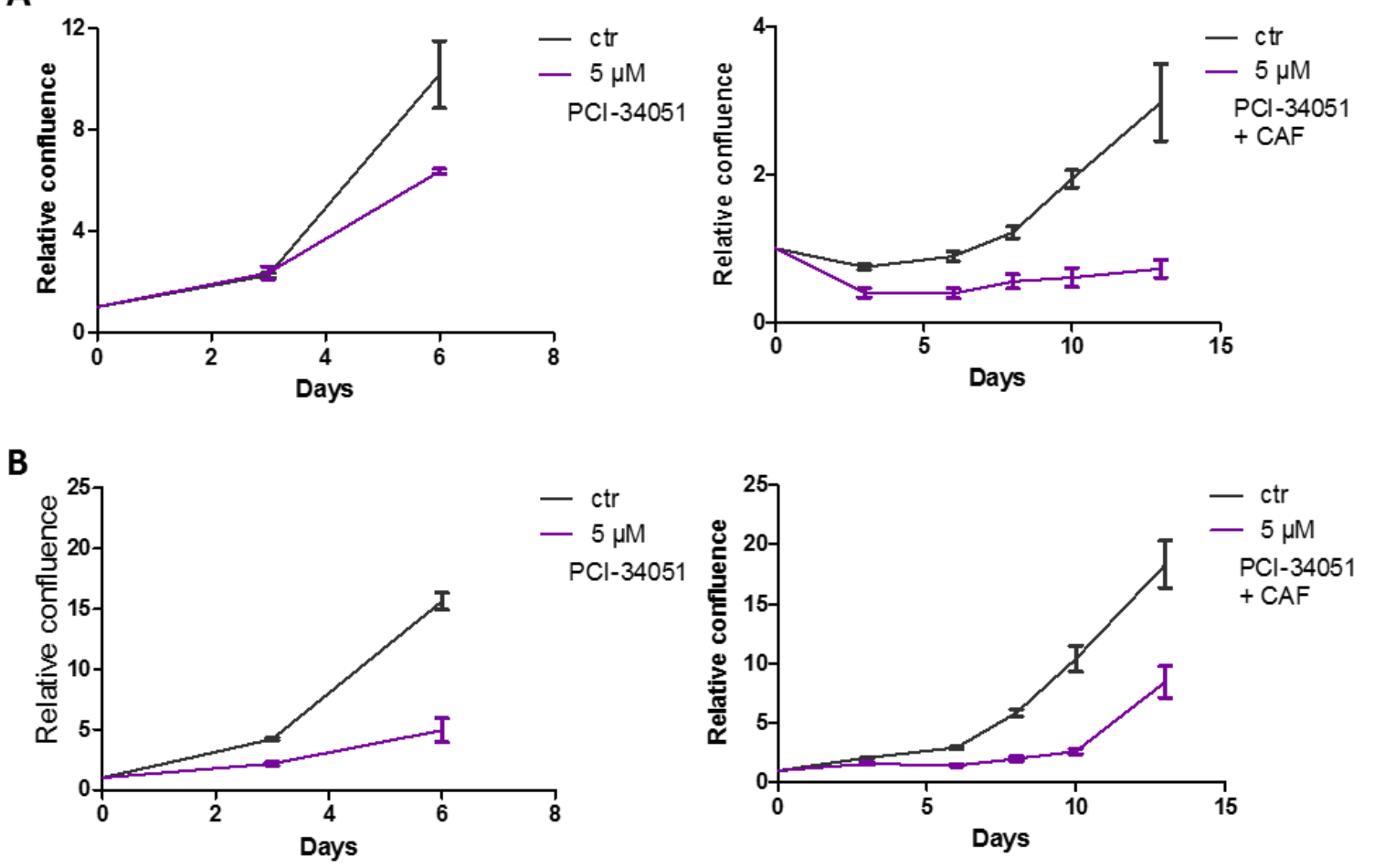

Figure II 4. Suppression of cell growth upon HDAC8 inhibition in murine TNBC cell lines. $A$ and

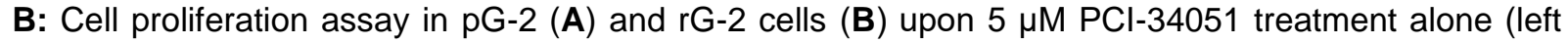
panel) or combined with 48 hours CAF-chemotherapy (1/32), followed by fresh medium culturing G-2 cells. Proliferation measurements determined by Celigo, $n=3+/-S E M$. 

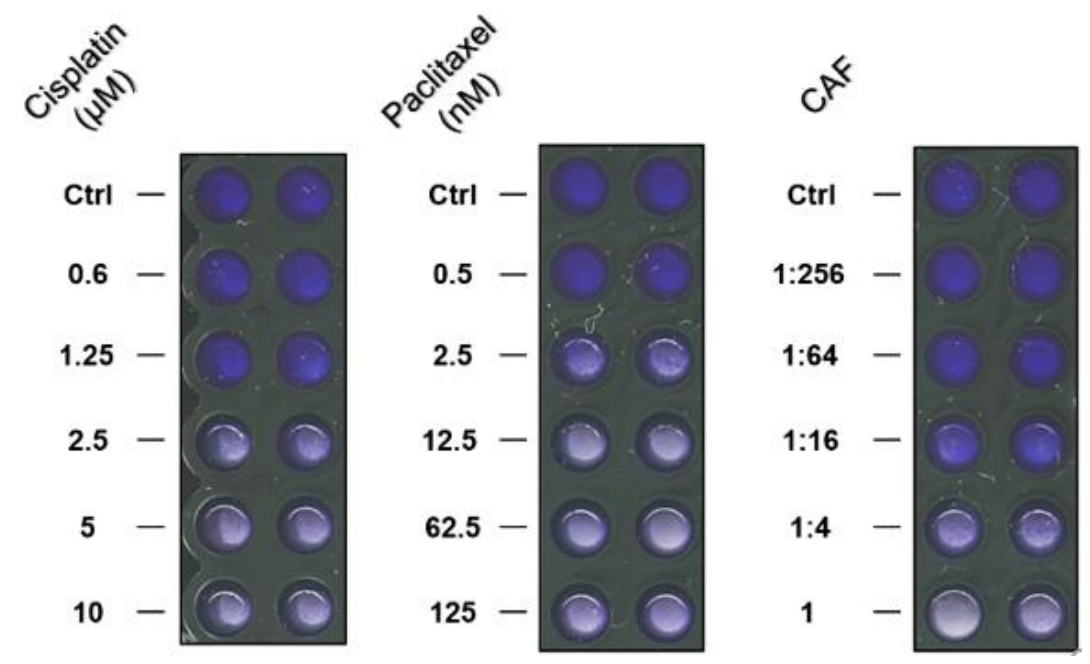

Figure II S1. Proliferation assay using crystal violet staining upon different chemotherapy concentrations in HCC1806 cell line. Cells were treated with chemotherapy 24 hours after seeding and were allowed to grow over 1 week. Concentration [1] for CAF is representing $0.5 \mu \mathrm{g} / \mathrm{ml}$ cyclophosphamide, $10 \mu \mathrm{g} / \mathrm{ml}$ doxorubicin, and $10 \mu \mathrm{g} / \mathrm{ml}$ 5-FU combination.

\section{Discussion}

TNBC is one of the most difficult cancers to treat, due to its heterogeneity and high cancer relapse rate (World Health Organisation, 2018). EMT was identified as a mechanism involved in overcoming cytotoxic treatments in TNBC cancer cells. EMT is cellular process modulated through epigenetic modifiers, like HDACs (R. Chang, You, \& Zhou, 2013; Lei et al., 2010). Despite first promising results, where all analyzed HDACs $(-4,-7,-8)$ affected proliferation of TNBC cells, HDAC4 and -7 loss did not switch cellular phenotype from mesenchymal to epithelial state. Additionally, inhibition of HDAC4 and -7 with or without chemotherapy combination did not synergize. Instead, among HDACs, the most promising anticancer target in TNBC seems to be HDAC8. Loss of HDAC8 activity lead to proliferation impairment in mouse and human TNBC, in vitro, that was also shown in neuroblastoma (Rettig et al., 2015). In our project we used PCl-34051 as a commercially available selective HDAC8 inhibitor, that is recommended for T-cell lymphoma and leukemia cells (Balasubramanian et al., 2008). Interestingly it showed cell survival impairment alone and increased sensitivity in 
combination with CAF-chemotherapy treatment. It would be intriguing to validate this effect in combination with other chemotherapy agents, like paclitaxel and cisplatin, where EMT regulation was increased even more than in the case of CAF. We could observe that only HDAC8 loss but not HDAC4 or HDAC7 loss regulated EMT state. For further studies one could determine what are the EMT changes upon HDAC8 inhibition in combination with chemotherapy treatment. Nowadays, pan-HDACs inhibitors are the most commonly studied (Singh et al., 2018). Despite the promising in vitro and in vivo studies, inhibiting most of the HDAC family can bring unwanted side effects among patients (Subramanian et al., 2010). The development of selective HDAC inhibitors is a key issue in the clinic. The results of our study support the hypothesis that HDAC8 inhibitor could represent a promising approach to sensitize or re-sensitize TNBCs to conventional cytotoxic anti-cancer therapies, where therapy options are limited. 


\section{General discussion}

\subsection{PRC2/EZH2 in TNBC progression}

More than $80 \%$ of breast cancer cases can be resected surgically, with $50 \%$ chance of cancer relapse among those patients (World Health Organization, 2006). Surgery can be followed by adjuvant chemotherapy to prevent cancer recurrence. Triple-negative breast cancer (TNBC) lacks targeted therapy options, consequently we wanted to elucidate TNBC invasion pathways upon chemotherapy as it is one of the most common therapy in TNBC patients (Wahba \& El-Hadaad, 2015). In this project we are focused on TNBC, however similar mechanism of cancer cell escape due to chemotherapy treatment can be found in other cancers. Therefore, we aimed that our results could be applied in other cancer systems.

Cytotoxic stimuli enhances epigenetic alterations to gain self-renewal, mesenchymal and metastatic properties in tumor cells (Easwaran, 2014). In many studies, it was shown that $\mathrm{PRC2} / \mathrm{EZH} 2$ plays a critical role in cancer progression and metastasis. Unsurprisingly, series of small molecule inhibitors of EZH2 have been developed. The methyltransferase EZH2 with its catalytic SET domain, transfers a methyl group from methyl donor, SAM (S-adenosyl-methionine) to lysine 27 on Histone H3 (H3K27). EZH2 inhibitors, blocking its SET domain, such as EPZ-6438, GSK2816126 and CPI-1205 are used, among others in phase II clinical trials in nonHodgkin lymphoma (Gulati, 2018). However, EZH2-inhibition-based clinical trials can result in therapeutic response failure ( NCT02082977, 2019), necessitating a greater attention for anti-EZH2 therapeutic approaches, maximizing benefits for cancer patients. In our project, CAF-chemotherapy leads to an epigenetic dysregulation in

TNBC cells. To our surprise, we observed unexpected PRC2 components loss favoring better survival of TNBC cells. Growing number of studies confirm our data, resulting in raising concerns about EZH2 as anti-cancer target. (Völkel et al., 2015).

To this date, TNBC patient cases remain a huge challenging clinic hurdle. Many trials on patients failed after EZH2 inhibition therapy. Treatment of some patients with Non-Hodgkin Lymphoma (NHL) during Phase I clinical trial, with EPZ6438 (small molecule EZH2 inhibitor) resulted in cancer recurrence (Italiano et al., 2018). In 
malignant myeloid diseases, loss-of-function EZH2 mutations were identified. They determined that loss of $\mathrm{EZH} 2$ and reduced $\mathrm{H} 3 \mathrm{~K} 27 \mathrm{me} 3$ level were associated with neoplastic disorders and leukemia progression (Muto et al., 2013). Interestingly, in hypoxic conditions, hypoxia-inducible factor 1 (HIF1- $\alpha$ ) induction leads to PRC2 inactivation. Released EZH2 from the complex, cooperates with Forkhead box M1 (FoxM1) leading to direct MMPs promoters regulation and TNBC invasion (Mahara et al., 2016)

TNBC shares molecular similarities with high-grade serous ovarian tumors (Bell et al., 2011; Wang et al., 2012). Based on TCGA data, both display frequent p53 mutations, $B R C A 1$ inactivation, $R B 1$ loss and $C M Y C$ amplification correlating with TNBC features. We observed that upon EZH2 inhibition TNBC cancer cells can progress and induce an even worse prognosis than before treatment. In our studies we show similar cell behavior of TNBC (pG-2 and MDA-MB-468) cells such as in colon cancer (HCT116 and HT-29) and bile duct (EGI-1 and TFK-1) carcinoma cell lines, where EZH2 inhibition lead to better cell growth. As we presented, TCGA-based data on TNBC patients indicates low survival rate in cancers with low EZH2 expression. Thus, it seems that the regulation through PRC2/EZH2 could be subtype-specific or context-dependent. Those data suggest a novel strategy in the treatment finding: it is indeed attractive to hypothesize that the same or very similar molecular patterns within cancers or their subtypes, could offer common clinical approach for category of patients with low EZH2 expression in cancer cells (Figure 12). 


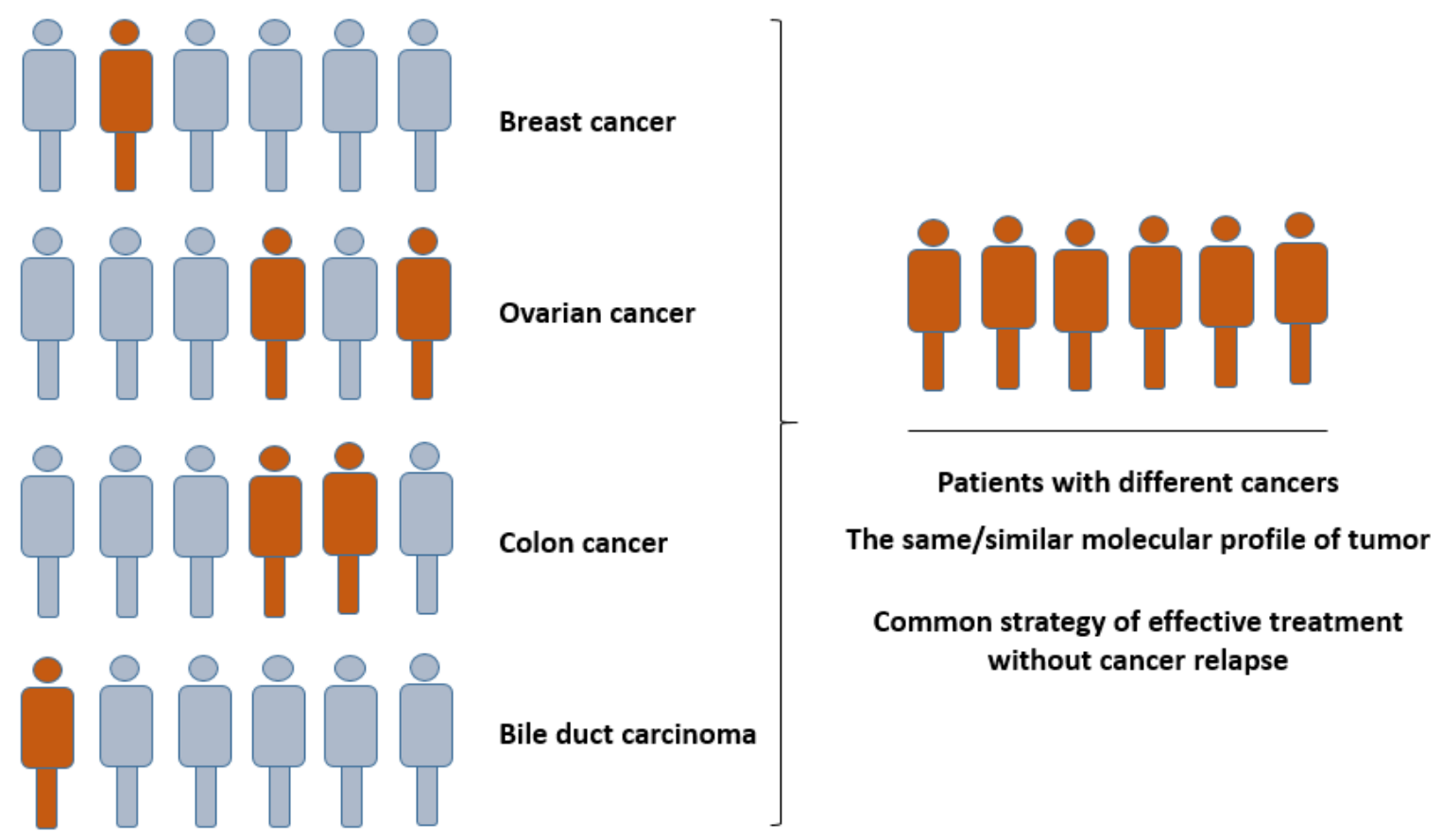

Figure 12: A scheme of the patients with distinct cancer types but similar treatment strategy and response. Orange indicates patient with common anti-cancer therapy strategy, grey reflects the patient with response to different anti-cancer treatment. Selected population of patients with distinct cancers benefited from the same clinical approach (orange, right).

\subsection{NFATc1 as a potential PRC2/EZH2-dependent driver of TNBC progression}

PRC2/EZH2-dependent activity is an important mechanism to repress gene transcription. Our data shows that $\mathrm{PRC2/EZH2} \mathrm{is} \mathrm{downregulated} \mathrm{upon} \mathrm{chemotherapy.}$ Under cytotoxic stimuli, decreased PRC2/EZH2 activity is likely to lead to the activation of previously repressed genes, being crucial for cancer cell fate. Loss of H3K27me3 and gain of $\mathrm{H} 3 \mathrm{~K} 27 \mathrm{ac}$ on promoter regions can directly activate targeted genes (Grimaldi et al., 2011). As chemotherapy is the most common way to treat TNBC displaying the highest response rate among breast cancer patients, we wanted to explore the mechanism that drive cell survival followed by chemotherapy. We identified Nfatc1, Wnt9a, Gli2 and Klf4 as the most relevant PRC2/EZH2-regulated genes that could be involved in chemotherapy resistance.

A number of studies have shown that NFATc1 favors cancer progression. For instance, overexpression or constitutively active NFATs are commonly linked with 
cancer progression involving angiogenesis and migration (Qin et al., 2014). The NFAT/calcineurin pathway is associated with increased invasion of mammary tumor cells (Yoeli-Lerner et al., 2009), as seen also in our TNBC system. Interestingly, breast cancer subtype-based gene expression analysis revealed that NFAT-related pathway is more frequent in TNBC in comparison to non-TNBC patients (Tran Quang et al., 2015). In our study, we showed that EZH2 is negatively correlated with NFATc1 in vitro and in vivo. NFATC1 loss lead to impaired cell proliferation and migration in our WAP$T$ in vitro system, indicating the involvement of NFATc1 in TNBC progression. Additionally, cell death in human TNBC cell line upon NFATC1 depletion suggests that NFATc1 plays essential role in cancer survival. In our data, we observed enrichment for calcineurin pathway in CAF-treated cells involving deregulation of NFATc1.

Intriguingly, NFATc1 was found as a promising anti-leukemia target. For instance, in Chronic Lymphocytic Leukemia (CLL), CsA- and FK-506-mediated NFATc1 inhibition induced apoptosis of CLL cells (Wolf et al., 2014). In Acute Myeloid Leukemia (AML) NFATc1 was proposed as a therapeutic target to overcome resistance (Metzelder et al., 2015). These studies and many other publications regarding blood cancers strongly support our results in TNBC (Pham et al., 2010). As mentioned previously, PRC2/EZH2 loss-of-function was indicated to enhance cancer progression in leukemia and lymphoma. This finding brings a potential into therapy of blood cancers and TNBC, where PRC2/EZH2-mediated NFATC1 regulation could play fundamental role in overcoming cancer progression (Figure 13).

Based on our results, $\mathrm{PRC2} / \mathrm{EZH} 2-$ dependent NFATC1 regulation can modulate cancer cell behavior through EMT. In previous studies, using the WAP-T model in vivo and in vitro, TGF- $ß$ pathway activation was shown to increase aggressiveness of WAPT tumor cells by inducing EMT (Maenz et al., 2015). Moreover, the group of Hessmann demonstrated a synergy between NFATC1 and TGFß1 signaling inducing pancreatic cancer progression by inhibiting apoptosis and growth arrest (Hasselluhn et al., 2019). TGFß1 signaling is also increased upon CAF treatment of G-2 cells (RNA-seq analyses, data not shown). It can be therefore hypothesized that both pathways cooperate with each other to stimulate cancer cell survival. Further studies would be necessary to prove this idea. In our studies, we determined that NFATc1 activity 
contributes to the more mesenchymal cell properties. Loss or inhibition of NFATc1 reversed this process, resulting in an increased epithelial cell fraction. These results are in line with former observations that NFATc1 is involved in the regulation of epithelial-to-mesenchymal plasticity and stemness (Gould et al., 2016) It is therefore tempting to speculate that reversal of an invasive phenotype via NFAT inhibition may represent a clinically beneficial approach for TNBC patients.

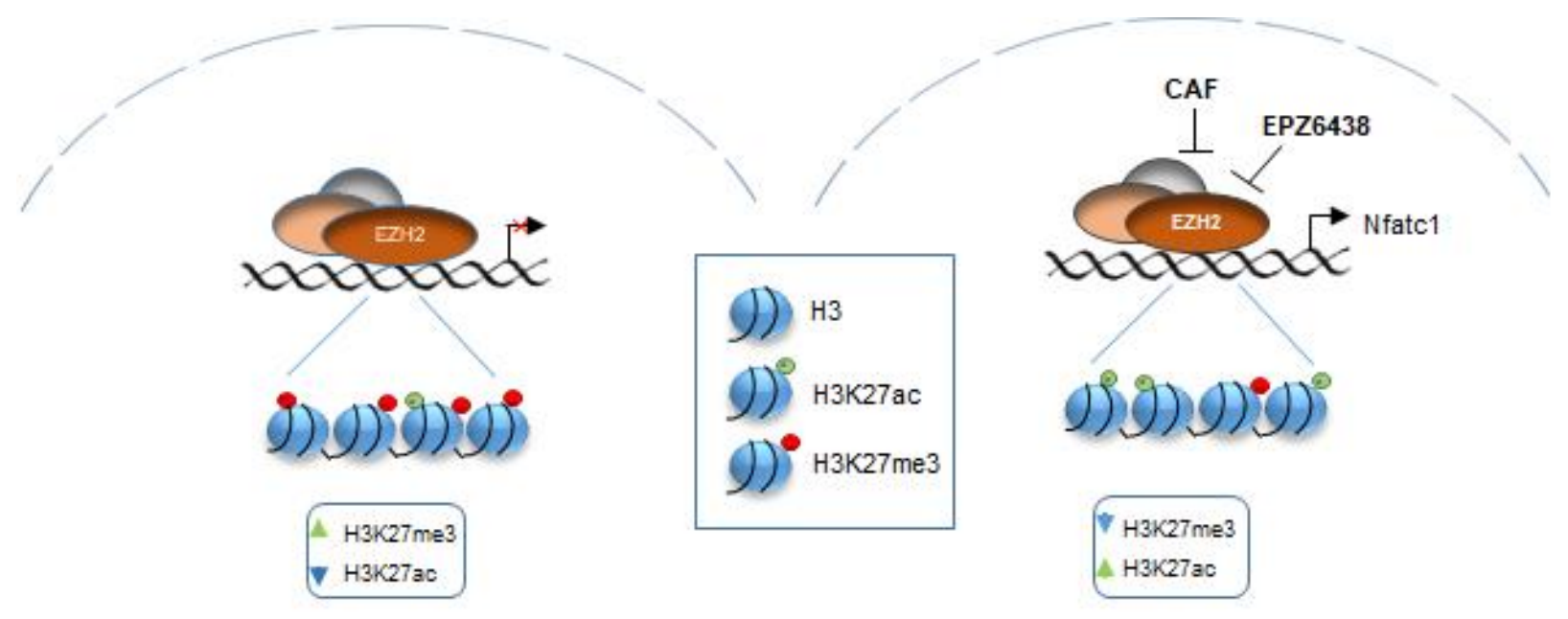

Figure 13: $\mathrm{PRC2} / \mathrm{EZH} 2$ downregulation upon CAF-chemotherapy or EPZ-6438 treatment leading to loss of H3K27me3 and gain of H3K27ac activating NFATC1.

\subsection{Gli2, KIf4, Wnt9a in cancer}

Apart of NFATc1, other factors were identified as being under control of the PRC2 repressive activity. One of these is Gli2, a transcription factor involved in hedgehog (Hh) pathway (Méthot \& Basler, 2001). Many studies support contribution of Hh signaling in cancer progression (Cannonier et al., 2016; D. Huang et al., 2018; N. $\mathrm{Li}$ et al., 2018). Gli2 leads to increased invasiveness, migration, angiogenesis and drug resistance in breast cancer (Atwood et al., 2015; Gupta et al. 2015; Han et al., 2016). Interestingly, its the most significant pro-tumorigenic function was reported in TNBC (Habib \& O'Shaughnessy, 2016). Gli2 is potential PRC2/EZH2-mediated factor involved in TNBC progression. Hh along with Wnt and TGF- $\beta$ signaling contributes to 
EMT and increased stemness capacity in breast, ovarian and pancreatic cancer (Morel et al., 2008; Noubissi et al., 2018; Scheel et al., 2011). Additionally, the Hh pathway is associated with drug resistance where its enrichment was observed in cancer cells resistant to paclitaxel and doxorubicin (Narita et al., 2008; Zhou et al., 2012). These findings support our results and suggest that PRC2-dependent Gli2 regulation could be additional or cooperating mechanism with PRC2-NFATc1 pathway leading to TNBC cell survival.

Together with hedgehog pathway, Wnt/beta-catenin signaling pathway was reported to promote cell proliferation and stemness maintenance (Mullor et al., 2001; Noubissi et al., 2018). Wnt/beta-catenin can lead to drug resistance and cell immortality through upregulation of MMP7 and hTERT, respectively. Wnt9a was identified as a major player in colon tumorigenesis (Bhattacharyya, Feferman, \& Tobacman, 2014). There is no data describing Wnt9a in TNBC progression. However, based on our data Wnt9a could be interesting gene candidate to investigate in chemotherapy resistance in TNBC.

Apart of NFATC1, Gli2 and Wnt9a, we identified Klf4 as a gene regulated upon chemotherapy. Kruppel-like factor 4 (KIf4) plays diverse functions in diseases (Ghaleb \& Yang, 2017). Klf4 activation by loss of EZH2 and H3K27me3 upon cytotoxic stimuli, could be one of the players inducing pluripotency of TNBC cells. In Mouse embryonic fibroblasts (MEFs), it was identified that calcineurin can be activated via overexpression of Klf4 (Khodeer \& Era, 2017). In TNBC, calcineurin phosphatase induction could potentially dephosphorylate NFATc1 and enhance even more its oncogenic function, additionally to PRC2 regulation.

\subsection{NFATC1 and HDAC inhibition in TNBC in the clinic}

Our studies indicated NFATc1 (Chapter I) and HDAC8 (Chapter II) as the upregulated factors upon chemotherapy treatment in TNBC cells. Based on our findings, HDAC8 appears to have prominent role in TNBC progression, where its inhibition alone impairs cancer cell growth with sensitization effect upon combination with chemotherapy. NFATC1 and HDAC8 are involved in TNBC cancer progression by 
promoting EMT. NFATC1 and HDAC8 loss or inhibition leads to decreased proliferation growth rate. Subsequently, NFATC1 and HDAC8 inhibition could bring therapeutical benefits for TNBC patients. As we showed in our project, HDAC8 inhibition via PCI34051 have anti-TNBC potential that was also observed in T-cell lymphomas where PCl-34051 treatment induced cell apoptosis (Balasubramanian et al., 2008). Additionally, we propose inhibition of HDAC8 in combination with other drug as an alternative for cytotoxic pan-HDACs inhibition. Despite several promising pan-HDAC inhibitors, FDA-approved or undergoing clinical trials, can give unwanted side effects, strongly affecting patient's life. In contrast, isoform-specific HDAC inhibitor could offer impairment of cancer progression with beneficial effect for patient survival. As epigenetic mechanisms are reversible, anti-tumorigenic effect of HDAC8 inhibition could be used as epigenetic therapy supporting NFATc1 blockade bringing effective therapeutic result. Thus, for further investigation, it would be interesting to check the effect of simultaneous inhibition of NFATc1 and HDAC8 that could synergistically impair cancer progression. This proposed therapy solution should be limited to particular category of patients. As it was discussed previously, in most of the cancers EZH2 was indicated as an oncogenic factor. The downregulated PRC2/EZH2 activity as a novel mechanism in cancer progression, should be further investigated among cancers. This information could imply molecular changes, such as NFAT pathway regulation.

In our project we propose that upregulation of NFATc1 and HDAC8 in TNBC could emerge as essential therapeutic targets. Therefore, selective anticancer drug combination involving NFATC1 and HDAC8 inhibition might be suggested as a novel anti-TNBC approach for further investigation.

In summary, we have investigated the novel landmark in TNBC survival to chemotherapy stimuli. We demonstrated that in chemotherapy-treated TNBC cells, loss of PRC2/EZH2 leads to NFATc1 activation initiating an EMT process and fostering cancer progression (Figure 13). Since many studies have shown critical function of NFATc1 in tumor progression, drug resistance and metastasis, NFATc1 inhibition may represent a potential strategy to overcome chemotherapy resistance in cancer. 
Additional investigation on NFATC1 function in TNBC progression will be needed in the future. Moreover, examination of the function of other PRC2/EZH2-dependent factors (Gli2, Wnt9a, Klf4), their importance in chemotherapy resistance and relationship with NFAT pathway could be interesting. Additionally, epigenetic PRC2/EZH2-dependent mechanism could potentially interplay with HDACs function inducing pro-tumorigenic pathways. Our findings suggest NFATc1 and HDAC8 as major regulators of TNBC progression that could be proposed for combinatory therapy, where NFATc1 and HDAC8 inhibition brings novel therapeutical approach. 


\section{Bibliography}

A Study to Investigate the Safety, Pharmacokinetics, Pharmacodynamics and Clinical Activity of GSK2816126 in Subjects With Relapsed/Refractory Diffuse Large B Cell Lymphoma, Transformed Follicular Lymphoma, Other Non-Hodgkin's Lymphomas, Solid Tumors and . (2019). https://doi.org/NCT02082977

Agger, K., Cloos, P. A. C., Christensen, J., Pasini, D., Rose, S., Rappsilber, J., ... Helin, K. (2007). UTX and JMJD3 are histone H3K27 demethylases involved in HOX gene regulation and development. Nature. https://doi.org/10.1038/nature06145

Antonysamy, S., Condon, B., Druzina, Z., Bonanno, J. B., Gheyi, T., Zhang, F., ... Luz, J. G. (2013). Structural context of disease-associated mutations and putative mechanism of autoinhibition revealed by X-Ray crystallographic analysis of the EZH2-SET domain. PLOS ONE, 8(12), 1-15. https://doi.org/10.1371/journal.pone.0084147

Aramburu, J., Yaffe, M. B., López-Rodríguez, C., Cantley, L. C., Hogan, P. G., \& Rao, A. (1999). Affinity-driven peptide selection of an NFAT inhibitor more selective than cyclosporin A. Science. https://doi.org/10.1126/science.285.5436.2129

Arents, G., Burlingame, R. W., Wang, B. C., Love, W. E., \& Moudrianakis, E. N. (1991). The nucleosomal core histone octamer at 3.1 A resolution: a tripartite protein assembly and a left-handed superhelix. Biochemistry, 88(22), 1014810152. Retrieved from

http://www.ncbi.nlm.nih.gov/pubmed/1946434\%0Ahttp://www.pubmedcentral.nih. gov/articlerender.fcgi?artid=PMC52885

Ariës, I. M., Bodaar, K., Karim, S. A., Chonghaile, T. N., Hinze, L., Burns, M. A., ... Gutierrez, A. (2018). PRC2 loss induces chemoresistance by repressing apoptosis in T cell acute lymphoblastic leukemia. Journal of Experimental Medicine. https://doi.org/10.1084/jem.20180570

Atwood, S. X., Sarin, K. Y., Whitson, R. J., Li, J. R., Kim, G., Rezaee, M., ... Tang, J. Y. (2015). Smoothened Variants Explain the Majority of Drug Resistance in Basal Cell Carcinoma. Cancer Cell. https://doi.org/10.1016/j.ccell.2015.02.002

Audia, J. E., \& Campbell, R. M. (2016). Histone Modifications and Cancer. Cold Spring Harbor Perspectives in Biology, 1-31.

B.Lehmann, J. Bauer, 1 X. Chen, M. Sanders, A. Chakravarthy, Y. S., \& Pietenpol, J. (2011). Identification of human triple-negative breast cancer subtypes and preclinical models for selection of targeted therapies. The Journal of Clinical InvestigationJournal of Clinical Investigation, 121(7). https://doi.org/10.1172/JCl45014DS1

Balasubramanian, S., Ramos, J., Luo, W., Sirisawad, M., Verner, E., \& Buggy, J. J. (2008). A novel histone deacetylase 8 (HDAC8)-specific inhibitor PCl-34051 induces apoptosis in T-cell lymphomas. Leukemia. 
https://doi.org/10.1038/leu.2008.9

Beals, C. R., Clipstone, N. A., Ho, S. N., \& Crabtree, G. R. (1997). Nuclear localization of NF-ATc by a calcineurin-dependent. Genes and Development, 11, 824-834.

Bell, D., Berchuck, A., Birrer, M., Chien, J., Cramer, D. W., Dao, F., ... Thomson, E. (2011). Integrated genomic analyses of ovarian carcinoma. Nature. https://doi.org/10.1038/nature10166

Berx, G., Nollet, F., Leeuwl, W. J. F. De, Vijverl, M. J. Van De, Cornelisse, C., \& Roy, F. Van. (1995). The prognostic significance of epithelial-mesenchymal transition in breast cancer, E-cadherin is a tumor/ invasion suppressor gene mutated in human lobular breast cancers. The EMBO Journal, 14(24), 6107-6115.

Bhattacharyya, S., Feferman, L., \& Tobacman, J. K. (2014). Increased expression of colonic Wnt9A through Sp1-mediated transcriptional effects involving arylsulfatase B, chondroitin 4-sulfate, and Galectin-3. Journal of Biological Chemistry. https://doi.org/10.1074/jbc.M114.561589

Bladt, F., Riethmacher, D., Isenmann, S., Aguzzi, A., \& Birchmeier, C. (1995). Essential role for the c-met receptor in the migration of myogenic precursor cells into the limb bud. Nature, Vol. 376, pp. 768-771. https://doi.org/10.1038/376768a0

Blanco, M. J., Moreno-Bueno, G., Sarrio, D., Locascio, A., Cano, A., Palacios, J., \& Nieto, M. A. (2002). Correlation of Snail expression with histological grade and lymph node status in breast carcinomas. Oncogene. https://doi.org/10.1038/sj.onc.1205416

Bolden, J. E., Peart, M. J., \& Johnstone, R. W. (2006). Anticancer activities of histone deacetylase inhibitors. Nature Reviews Drug Discovery. https://doi.org/10.1038/nrd2133

Bonnet, D., \& Dick, J. E. (1997). Human acute myeloid leukemia is organized as a hierarchy that originates from a primitive hematopoietic cell. Nature Medicine. https://doi.org/10.1038/nm0797-730

Boyer, L. A., Plath, K., Zeitlinger, J., Brambrink, T., Medeiros, L. A., Lee, T. I., ... Jaenisch, R. (2006). Polycomb complexes repress developmental regulators in murine embryonic stem cells. Nature. https://doi.org/10.1038/nature04733

Buisson, R., Dion-Côté, A. M., Coulombe, Y., Launay, H., Cai, H., Stasiak, A. Z., ... Masson, J. Y. (2010). Cooperation of breast cancer proteins PALB2 and piccolo BRCA2 in stimulating homologous recombination. Nature Structural and Molecular Biology. https://doi.org/10.1038/nsmb.1915

Cannonier, S. A., Gonzales, C. B., Ely, K., Guelcher, S. A., \& Sterling, J. A. (2016). Hedgehog and TGF $\beta$ signaling converge on Gli2 to control bony invasion and bone destruction in oral squamous cell carcinoma. Oncotarget. https://doi.org/10.18632/oncotarget.12584

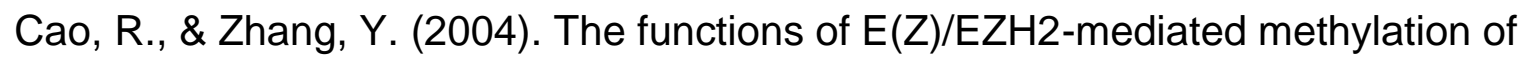


lysine 27 in histone H3. Current Opinion in Genetics and Development, 14(2), 155-164. https://doi.org/10.1016/j.gde.2004.02.001

Cardenas, H., Zhao, J., Vieth, E., Nephew, K. P., \& Matei, D. (2016). EZH2 inhibition promotes epithelial-to-mesenchymal transition in ovarian cancer cells.

Oncotarget, 7(51), 84453-84467. https://doi.org/10.18632/oncotarget.11497

Carey, L. A., Dees, E. C., Sawyer, L., Gatti, L., Moore, D. T., Collichio, F., ... Perou, C. M. (2007). The triple negative paradox: Primary tumor chemosensitivity of breast cancer subtypes. Clinical Cancer Research, 13(8), 2329-2334. https://doi.org/10.1158/1078-0432.CCR-06-1109

Chacón López-Muñiz, J. I., de la Cruz Merino, L., Gavilá Gregori, J., Martínez Dueñas, E., Oliveira, M., Seguí Palmer, M. A., ... López-Tarruella Cobo, S. (2019). SEOM clinical guidelines in advanced and recurrent breast cancer (2018). Clinical and Translational Oncology, 21(1), 31-45. https://doi.org/10.1007/s12094-018-02010-w

Chang, C., \& Hung, M. (2011). The role of EZH2 in tumour progression. 106(2), 243247. https://doi.org/10.1038/bjc.2011.551

Chang, R., You, J., \& Zhou, Q. (2013). Research advance on mechanism and application of HATs and HDACs in epithelial-mesenchymal transition of lung cancer. Chinese Journal of Lung Cancer. https://doi.org/10.3779/j.issn.10093419.2013.04.07

Chen, N. M., Neesse, A., Dyck, M. L., Steuber, B., Koenig, A. O., LubesederMartellato, C., ... Hessmann, E. (2017). Context-Dependent Epigenetic Regulation of Nuclear Factor of Activated T Cells 1 in Pancreatic Plasticity. Gastroenterology. https://doi.org/10.1053/j.gastro.2017.01.043

Chen, Y., Xie, D., Yin Li, W., Man Cheung, C., Yao, H., Chan, C. Y., ... Kung, H. F. (2010). RNAi targeting EZH2 inhibits tumor growth and liver metastasis of pancreatic cancer in vivo. Cancer Letters.

https://doi.org/10.1016/j.canlet.2010.05.003

Chien, Y.-C., Liu, L.-C., Ye, H.-Y., Wu, J.-Y., \& Yu, Y.-L. (2018). EZH2 promotes migration and invasion of triple-negative breast cancer cells via regulating TIMP2-MMP-2/-9 pathway. American Journal of Cancer Research.

Chiu, H. W., Yeh, Y. L., Wang, Y. C., Huang, W. J., Chen, Y. A., Chiou, Y. S., ... Wang, Y. J. (2013). Suberoylanilide Hydroxamic Acid, an Inhibitor of Histone Deacetylase, Enhances Radiosensitivity and Suppresses Lung Metastasis in Breast Cancer In Vitro and In Vivo. PLOS ONE. https://doi.org/10.1371/journal.pone.0076340

Chopra, V. S., Hendrix, D. A., Core, L. J., Tsui, C., Lis, J. T., \& Levine, M. (2011). The Polycomb Group Mutant esc Leads to Augmented Levels of Paused Pol II in the Drosophila Embryo. Molecular Cell. https://doi.org/10.1016/j.molcel.2011.05.009

Colzani, E., Johansson, A. L. V., Liljegren, A., Foukakis, T., Clements, M., Adolfsson, J., ... Czene, K. (2014). Time-dependent risk of developing distant metastasis in breast cancer patients according to treatment, age and tumour characteristics. 
British Journal of Cancer. https://doi.org/10.1038/bjc.2014.5

D. Koboldt, R. Fulton, M. McLellan, H. Schmidt, J. Kalicki-Veizer, J. McMichael, L.

Fulton, D. Dooling, L. Mardis, R. Wilson, A. Ally, M. Balasundaram, Y. Butterfi, R. C. (2012). Comprehensive molecular portraits of human breast tumours. Nature, 490(7418), 61-70. https://doi.org/10.1038/nature11412.Comprehensive

Dalerba, P., Cho, R. W., \& Clarke, M. F. (2007). Cancer Stem Cells: Models and Concepts. Annual Review of Medicine.

https://doi.org/10.1146/annurev.med.58.062105.204854

Davar, D., H. Beumer, J., Hamieh, L., \& Tawbi, H. (2012). Role of PARP Inhibitors in Cancer Biology and Therapy. Current Medicinal Chemistry. https://doi.org/10.2174/092986712802002464

Dawson, M. A., Prinjha, R. K., Dittmann, A., Giotopoulos, G., Bantscheff, M., Chan, W. I., ... Kouzarides, T. (2011). Inhibition of BET recruitment to chromatin as an effective treatment for MLL-fusion leukaemia. Nature. https://doi.org/10.1038/nature10509

Dean, M., Fojo, T., \& Bates, S. (2005). Tumour stem cells and drug resistance. Nature Reviews Cancer. https://doi.org/10.1038/nrc1590

Denisenko, O., Shnyreva, M., Suzuki, H., \& Bomsztyk, K. (1998). Point Mutations in the WD40 Domain of Eed Block Its Interaction with Ezh2. Molecular and Cellular Biology. https://doi.org/10.1128/mcb.18.10.5634

Dhankhar, R., Vyas, S. P., Jain, A. K., Arora, S., Rath, G., \& Goyal, A. K. (2010). Advances in novel drug delivery strategies for breast cancer therapy. Artificial Cells, Blood Substitutes, and Biotechnology. https://doi.org/10.3109/10731199.2010.494578

Di Croce, L., \& Helin, K. (2013). Transcriptional regulation by Polycomb group proteins. Nature Structural and Molecular Biology. https://doi.org/10.1038/nsmb.2669

Duvic, M., Talpur, R., Ni, X., Zhang, C., Hazarika, P., Kelly, C., ... Frankel, S. R. (2007). Phase 2 trial of oral vorinostat (suberoylanilide hydroxamic acid, SAHA) for refractory cutaneous T-cell lymphoma (CTCL). Blood. https://doi.org/10.1182/blood-2006-06-025999

Easwaran, H., Tsai, H. C., \& Baylin, S. B. (2014). Cancer Epigenetics: Tumor Heterogeneity, Plasticity of Stem-like States, and Drug Resistance. Molecular Cell. https://doi.org/10.1016/j.molcel.2014.05.015

Echeverria, G. V., Ge, Z., Seth, S., Zhang, X., Jeter-Jones, S., Zhou, X., ... PiwnicaWorms, H. (2019). Resistance to neoadjuvant chemotherapy in triple-negative breast cancer mediated by a reversible drug-tolerant state. Science Translational Medicine, 11(488), eaav0936. https://doi.org/10.1126/scitransImed.aav0936

Ferlay, J., Shin, H. R., Bray, F., Forman, D., Mathers, C., \& Parkin, D. M. (2010). Estimates of worldwide burden of cancer in 2008: GLOBOCAN 2008. International Journal of Cancer, 127(12), 2893-2917. 
https://doi.org/10.1002/ijc.25516

Fisher, B., Anderson, S., Bryant, J., Margolese, R. G., Deutsch, M., Fisher, E. R., ... Wolmark, N. (2002). Twenty-year follow-up of a randomized trial comparing total mastectomy, lumpectomy, and lumpectomy plus irradiation for the treatment of invasive breast cancer. The New England Journal of Medicine, 347(16), 12331241. https://doi.org/10.1056/NEJMoa022152

Flanagan, W. M., Corthésy, B., Bram, R. J., \& Crabtree, G. R. (1991). Nuclear association of a T-cell transcription factor blocked by FK-506 and cyclosporin A. Nature, Vol. 352, pp. 803-807. https://doi.org/10.1038/352803a0

Gan, L., Yang, Y., Li, Q., Feng, Y., Liu, T., \& Guo, W. (2018). Epigenetic regulation of cancer progression by EZH2: From biological insights to therapeutic potential. Biomarker Research, 6(1), 1-10. https://doi.org/10.1186/s40364-018-0122-2

Garmpis, N., Damaskos, C., Garmpi, A., Kalampokas, E., Kalampokas, T., Spartalis, E., ... Dimitroulis, D. (2017). Histone deacetylases as new therapeutic targets in triple-negative breast cancer: Progress and promises. Cancer Genomics and Proteomics. https://doi.org/10.21873/cgp.20041

Ghaleb, A. M., \& Yang, V. W. (2017). Krüppel-like factor 4 (KLF4): What we currently know. Gene. https://doi.org/10.1016/j.gene.2017.02.025

Gluz, O., Liedtke, C., Gottschalk, N., Pusztai, L., Nitz, U., \& Harbeck, N. (2009). Triple-negative breast cancer - Current status and future directions. Annals of Oncology, 20(12), 1913-1927. https://doi.org/10.1093/annonc/mdp492

Gluz, Oleg, Nitz, U. A., Harbeck, N., Ting, E., Kates, R., Herr, A., ... Mohrmann, S. (2008). Triple-negative high-risk breast cancer derives particular benefit from dose intensification of adjuvant chemotherapy: Results of WSG AM-01 trial. Annals of Oncology. https://doi.org/10.1093/annonc/mdm551

Gonçalves, H., Guerra, M. R., Duarte Cintra, J. R., Fayer, V. A., Brum, I. V., \& Bustamante Teixeira, M. T. (2018). Survival Study of Triple-Negative and NonTriple-Negative Breast Cancer in a Brazilian Cohort. Clinical Medicine Insights: Oncology, 12. https://doi.org/10.1177/1179554918790563

Gonzalez, M. E., Li, X., Toy, K., DuPrie, M., Ventura, A. C., Banerjee, M., ... Kleer, C. G. (2009). Downregulation of EZH2 decreases growth of estrogen receptornegative invasive breast carcinoma and requires BRCA1. Oncogene, 28(6), 843853. https://doi.org/10.1038/onc.2008.433

Gould, R., Bassen, D. M., Chakrabarti, A., Varner, J. D., \& Butcher, J. (2016). Population Heterogeneity in the Epithelial to Mesenchymal Transition Is Controlled by NFAT and Phosphorylated Sp1. PLoS Computational Biology. https://doi.org/10.1371/journal.pcbi.1005251

Gregoretti, I. V., Lee, Y. M., \& Goodson, H. V. (2004). Molecular evolution of the histone deacetylase family: Functional implications of phylogenetic analysis. Journal of Molecular Biology, 338(1), 17-31. https://doi.org/10.1016/j.jmb.2004.02.006 
Grimaldi, G., Christian, M., Steel, J. H., Henriet, P., Poutanen, M., \& Brosens, J. J. (2011). Down-regulation of the histone methyltransferase EZH2 contributes to the epigenetic programming of decidualizing human endometrial stromal cells. Molecular Endocrinology. https://doi.org/10.1210/me.2011-1139

Grossniklaus, U., \& Paro, R. (2014). Transcriptional Silencing by Polycomb-Group Proteins. Cold Spring Harbor Perspectives in Biology. https://doi.org/10.1101/cshperspect.a019331

Grunstein, M. (1997). Histone acetylation in chromatin structure and transcription. Nature. https://doi.org/10.1038/38664

Gulati, N., Béguelin, W., \& Giulino-Roth, L. (2018). Enhancer of zeste homolog 2 (EZH2) inhibitors. Leukemia and Lymphoma. https://doi.org/10.1080/10428194.2018.1430795

Gupta, P., Ranjan, A., Fofaria, N. M., \& Srivastava, S. K. (2015). Abstract 2256: GLI2 inhibition suppresses metastasis of HER2+ breast cancer. https://doi.org/10.1158/1538-7445.am2015-2256

Habib, J. G., \& O'Shaughnessy, J. A. (2016). The hedgehog pathway in triplenegative breast cancer. Cancer Medicine. https://doi.org/10.1002/cam4.833

Haffty, B. G., Yang, Q., Reiss, M., Kearney, T., Higgins, S. A., Weidhaas, J., ... Toppmeyer, D. (2019). Locoregional Relapse and Distant Metastasis in Conservatively Managed Triple Negative Early-Stage Breast Cancer. JOURNAL OF CLINICAL ONCOLOGY, 24(36). https://doi.org/10.1200/JCO.2006.06.5664

Han, B., Qu, Y., Yu-Rice, Y., Johnson, J., \& Cui, X. (2016). FOXC1-induced Gli2 activation: A non-canonical pathway contributing to stemness and anti-Hedgehog resistance in basal-like breast cancer. Molecular and Cellular Oncology. https://doi.org/10.1080/23723556.2015.1131668

Han, J., Lim, W., You, D., Jeong, Y., Kim, S., Lee, J. E., ... Park, S. (2019). Chemoresistance in the Human Triple-Negative Breast Cancer Cell Line MDAMB-231 Induced by Doxorubicin Gradient Is Associated with Epigenetic Alterations in Histone Deacetylase. Journal of Oncology. https://doi.org/10.1155/2019/1345026

Harbeck, N., Penault-Llorca, F., Cortes, J., Michael, G., Nehmat, H., Poortmans, P., ... Cardoso, F. (2019). Breast cancer. PRIMER, 5(66). https://doi.org/10.1016/S0140-6736(16)31891-8

Hasan, M., Leak, R. K., Stratford, R. E., Zlotos, D. P., \& Witt-Enderby, P. A. (2018). Drug conjugates-an emerging approach to treat breast cancer. Pharmacology Research and Perspectives, 6(4). https://doi.org/10.1002/prp2.417

Hasselluhn, M. C., Schmidt, G. E., Ellenrieder, V., Johnsen, S. A., \& Hessmann, E. (2019). Aberrant NFATc1 signaling counteracts TGF $\beta$-mediated growth arrest and apoptosis induction in pancreatic cancer progression. Cell Death and Disease. https://doi.org/10.1038/s41419-019-1682-2

He, A., Shen, X., Ma, Q., Cao, J., von Gise, A., Zhou, P., ... Pu, W. T. (2012). PRC2 
directly methylates GATA4 and represses its transcriptional activity. Genes and Development. https://doi.org/10.1101/gad.173930.111

Hennessy, B. T., Gonzalez-Angulo, A. M., Stemke-Hale, K., Gilcrease, M. Z., Krishnamurthy, S., Lee, J. S., ... Mills, G. B. (2009). Characterization of a naturally occurring breast cancer subset enriched in epithelial-to-mesenchymal transition and stem cell characteristics. Cancer Research. https://doi.org/10.1158/0008-5472.CAN-08-3441

Hennig, G., Behrens, J., Truss, M., Frisch, S., Reichmann, E., \& Birchmeier, W. (1995). PROGRESSION OF CARCINOMA-CELLS IS ASSOCIATED WITH ALTERATIONS IN CHROMATIN STRUCTURE AND FACTOR-BINDING AT THE E-CADHERIN PROMOTER IN-VIVO. Oncogene.

Hennighausen, L. (1990). The mammary gland as a bioreactor: Production of foreign proteins in milk. Protein Expression and Purification. https://doi.org/10.1016/1046-5928(90)90037-Y

Herranz, N., Pasini, D., Díaz, V. M., Francí, C., Gutierrez, A., Dave, N., ... Peiró, S. (2008). Polycomb complex 2 is required for E-cadherin repression by the Snail1 transcription factor. Molecular and Cellular Biology, 28(15), 4772-4781. https://doi.org/10.1128/MCB.00323-08

Hirukawa, A., Smith, H. W., Zuo, D., Dufour, C. R., Savage, P., Bertos, N., ... Muller, W. J. (2018). Targeting EZH2 reactivates a breast cancer subtype-specific antimetastatic transcriptional program. Nature Communications, 9(1). https://doi.org/10.1038/s41467-018-04864-8

Hogan, P. G., Hogan, P. G., Chen, L., \& Chen, L. (2003). Transcriptional regulation by calcium, calcineurin. Genes and Development, 17(18), 2205-2232. https://doi.org/10.1101/gad.1102703.GENES

Hsieh, C. L., Ma, H. P., Su, C. M., Chang, Y. J., Hung, W. Y., Ho, Y. S., ... Lin, R. K. (2016). Alterations in histone deacetylase 8 lead to cell migration and poor prognosis in breast cancer. Life Sciences. https://doi.org/10.1016/j.lfs.2016.02.092

Hu, S., Yu, L., Li, Z., Shen, Y., Wang, J., Cai, J., ... Wang, Z. (2010). Overexpression of EZH2 contributes to acquired cisplatin resistance in ovarian cancer cells in vitro and in vivo. Cancer Biology and Therapy. https://doi.org/10.4161/cbt.10.8.12913

Huang, D., Wang, Y., Xu, L., Chen, L., Cheng, M., Shi, W., ... Luo, S. (2018). GLI2 promotes cell proliferation and migration through transcriptional activation of ARHGEF16 in human glioma cells. Journal of Experimental and Clinical Cancer Research. https://doi.org/10.1186/s13046-018-0917-x

Huang, J. P., \& Ling, K. (2017). EZH2 and histone deacetylase inhibitors induce apoptosis in triple negative breast cancer cells by differentially increasing $\mathrm{H} 3$ Lys27 acetylation in the BIM gene promoter and enhancers. Oncology Letters. https://doi.org/10.3892/ol.2017.6912

Hutchinson, K. E., Nixon, M. J., Estrada, M. V, Sánchez, V., Sanders, M. E., Lee, T., 
... Arteaga, C. L. (2018). MYC and MCL1 Cooperatively Promote ChemotherapyResistant Breast Cancer Stem Cells via Regulation of Mitochondrial Oxidative Phosphorylation. Cell Metabolism, 26(4), 633-647.

https://doi.org/10.1016/j.cmet.2017.09.009.MYC

Im, J. Y., Lee, K. W., Won, K. J., Kim, B. K., Ban, H. S., Yoon, S. H., ... Won, M. (2016). DNA damage-induced apoptosis suppressor (DDIAS), a novel target of NFATc1, is associated with cisplatin resistance in lung cancer. Biochimica et Biophysica Acta - Molecular Cell Research. https://doi.org/10.1016/j.bbamcr.2015.10.011

Isakoff, S. J. (2010). Triple-negative breast cancer: Role of specific chemotherapy agents. Cancer Journal. https://doi.org/10.1097/PPO.0b013e3181d24ff7

Italiano, A., Soria, J. C., Toulmonde, M., Michot, J. M., Lucchesi, C., Varga, A., ... Ribrag, V. (2018). Tazemetostat, an EZH2 inhibitor, in relapsed or refractory Bcell non-Hodgkin lymphoma and advanced solid tumours: a first-in-human, openlabel, phase 1 study. The Lancet Oncology, 19(5), 649-659. https://doi.org/10.1016/S1470-2045(18)30145-1

Izumiya, M., Kabashima, A., Higuchi, H., Igarashi, T., Sakai, G., lizuka, H., ... Hibi, T. (2012). Chemoresistance is associated with cancer stem cell-like properties and epithelial-to-mesenchymal transition in pancreatic cancer cells. Anticancer Research, 32(9), 3847-3853.

Jannasch, K., Wegwitz, F., Lenfert, E., Maenz, C., Deppert, W., \& Alves, F. (2015). Chemotherapy of WAP-T mouse mammary carcinomas aggravates tumor phenotype and enhances tumor cell dissemination. International Journal of Cancer. https://doi.org/10.1002/ijc.29369

Jenuwein, T., \& Allis, C. D. (2001). Translating the histone code. Science, 293(5532), 1074-1080. https://doi.org/10.1126/science.1063127

Jung, H., Jun, S., Lee, M., Kim, H., Wang, X., Ji H., McCrea, P., Park, J. (2013). PAF and $\mathrm{EZH} 2$ induce $\mathrm{Wnt} / \beta$-catenin signaling hyperactivation. Molecular Cell, 52(2), 193-205. https://doi.org/10.1016/j.molcel.2013.08.028.PAF

Kalluri, R., \& Weinberg, R. A. (2009). The basics of epithelial-mesenchymal transition. Journal of Clinical Investigation. https://doi.org/10.1172/JCI39104

Kao, C., Huang, J., Wu, S. Q., Hauser, P., \& Reznikoff, C. A. (1993). Role of sv40 t antigen binding to prb and p53 in multistep transformation in vitro of human uroepithelial cells. Carcinogenesis. https://doi.org/10.1093/carcin/14.11.2297

Kashiwagi, S., Yashiro, M., Takashima, T., Aomatsu, N., Ikeda, K., Ogawa, Y., ... Hirakawa, K. (2011). Advantages of adjuvant chemotherapy for patients with triple-negative breast cancer at Stage II : usefulness of prognostic markers Ecadherin and Ki67. Breast Cancer Research, 13(6), R122. https://doi.org/10.1186/bcr3068

Katoh, N., Kuroda, K., Tomikawa, J., Ogata-kawata, H., \& Ozaki, R. (2018). Reciprocal changes of H3K27ac and H3K27me3 at the promoter regions of the critical genes for endometrial decidualization. Epigenomics, 10, 1243-1257. 
Kawahara, T., Kashiwagi, E., Ide, H., Li, Y., Zheng, Y., Miyamoto, Y., ... Miyamoto, H. (2015). Cyclosporine A and tacrolimus inhibit bladder cancer growth through down-regulation of NFATc1. Oncotarget. https://doi.org/10.18632/oncotarget.2750

Kawai, H., Li, H., Avraham, S., Jiang, S., \& Avraham, H. K. (2003). Overexpression of histone deacetylase HDAC1 modulates breast cancer progression by negative regulation of estrogen receptor $\alpha$. International Journal of Cancer. https://doi.org/10.1002/ijc. 11403

Khodeer, S., \& Era, T. (2017). Identifying the Biphasic Role of Calcineurin/NFAT Signaling Enables Replacement of Sox2 in Somatic Cell Reprogramming. Stem Cells. https://doi.org/10.1002/stem.2572

Kiesslich, T., Pichler, M., \& Neureiter, D. (2012). Epigenetic control of epithelialmesenchymal-transition in human cancer. Molecular and Clinical Oncology, 1(1), 3-11. https://doi.org/10.3892/mco.2012.28

Kikuchi, J., Koyama, D., Wada, T., Izumi, T., Hofgaard, P. O., Bogen, B., \& Furukawa, Y. (2015). Phosphorylation-mediated EZH2 inactivation promotes drug resistance in multiple myeloma. Journal of Clinical Investigation. https://doi.org/10.1172/JCl80325

Kim, E., Kim, M., Woo, D. H., Shin, Y., Shin, J., Chang, N., ... Lee, J. (2013). Phosphorylation of EZH2 Activates STAT3 Signaling via STAT3 Methylation and Promotes Tumorigenicity of Glioblastoma Stem-like Cells. Cancer Cell. https://doi.org/10.1016/j.ccr.2013.04.008

Kim, K. H., \& Roberts, C. W. M. (2016). Targeting EZH2 in cancer. Nature Medicine. https://doi.org/10.1038/nm.4036

Kleer, C. G., Cao, Q., Varambally, S., Shen, R., Ota, I., Tomlins, S. A., ... Chinnaiyan, A. M. (2003). EZH2 is a marker of aggressive breast cancer and promotes neoplastic transformation of breast epithelial cells. Proceedings of the National Academy of Sciences, 100(20), 11606-11611. https://doi.org/10.1073/pnas.1933744100

Krämer, O. H., Mahboobi, S., \& Sellmer, A. (2014). Drugging the HDAC6-HSP90 interplay in malignant cells. Trends in Pharmacological Sciences. https://doi.org/10.1016/j.tips.2014.08.001

Krishnamurthy, A., Dasari, A., Noonan, A. M., Mehnert, J. M., Lockhart, A. C., Leong, S., ... Lieu, C. H. (2018). Phase IB results of the rational combination of selumetinib and cyclosporin $A$ in advanced solid tumors with an expansion cohort in metastatic colorectal cancer. Cancer Research, 78(18), 5398-5407. https://doi.org/10.1158/0008-5472.CAN-18-0316

Krusche, C. A., Wülfing, P., Kersting, C., Vloet, A., Böcker, W., Kiesel, L., ... Alfer, J. (2005). Histone deacetylase-1 and -3 protein expression in human breast cancer: A tissue microarray analysis. Breast Cancer Research and Treatment. https://doi.org/10.1007/s10549-004-1668-2

Laemmli, U. K. (1970). Cleavage of structural proteins during the assembly of the 
head of bacteriophage T4. Nature. https://doi.org/10.1038/227680a0

Lajtha, L. G. (1967). Stem cells and their properties. Proceedings. Canadian Cancer Conference.

Le Roy, C., Deglesne, P. A., Chevallier, N., Beitar, T., Eclache, V., Quettier, M., ... Varin-Blank, N. (2012). The degree of BCR and NFAT activation predicts clinical outcomes in chronic lymphocytic leukemia. Blood. https://doi.org/10.1182/blood2011-12-397158

Lee, J. M., Lee, J. S., Kim, H., Kim, K., Park, H., Kim, J. Y., ... Baek, S. H. (2012). EZH2 Generates a Methyl Degron that Is Recognized by the DCAF1/DDB1/CUL4 E3 Ubiquitin Ligase Complex. Molecular Cell. https://doi.org/10.1016/j.molcel.2012.09.004

Lei, W., Zhang, K., Pan, X., Hu, Y., Wang, D., Yuan, X., ... Song, J. (2010). Histone deacetylase 1 is required for transforming growth factor- $\beta 1$-induced epithelialmesenchymal transition. International Journal of Biochemistry and Cell Biology. https://doi.org/10.1016/j.biocel.2010.05.006

Lenfert, E., Maenz, C., Heinlein, C., Jannasch, K., Schumacher, U., Pantel, K., ... Wegwitz, F. (2015). Mutant p53 promotes epithelial-mesenchymal plasticity and enhances metastasis in mammary carcinomas of WAP-T mice. International Journal of Cancer, 136(6), E521-E533. https://doi.org/10.1002/ijc.29186

Li, H., Handsaker, B., Wysoker, A., Fennell, T., Ruan, J., Homer, N., ... Durbin, R. (2009). The Sequence Alignment/Map format and SAMtools. Bioinformatics, 25(16), 2078-2079. https://doi.org/10.1093/bioinformatics/btp352

Li, N., Truong, S., Nouri, M., Moore, J., Al Nakouzi, N., Lubik, A. A., \& Buttyan, R. (2018). Non-canonical activation of hedgehog in prostate cancer cells mediated by the interaction of transcriptionally active androgen receptor proteins with Gli3. Oncogene. https://doi.org/10.1038/s41388-017-0098-7

$\mathrm{Li}, \mathrm{Y} .$, \& Seto, E. (2016). HDACs and HDAC inhibitors in cancer development and therapy. Cold Spring Harbor Perspectives in Medicine. https://doi.org/10.1101/cshperspect.a026831

Liedtke, C., Mazouni, C., Hess, K. R., André, F., Tordai, A., Mejia, J. A., .. Pusztai, L. (2008). Response to neoadjuvant therapy and long-term survival in patients with triple-negative breast cancer. Journal of Clinical Oncology. https://doi.org/10.1200/JCO.2007.14.4147

Liu, F., Zhu, Z., Mao, Y., Liu, M., Tang, T., \& Qiu, S. (2009). Inhibition of titanium particle-induced osteoclastogenesis through inactivation of NFATc1 by VIVIT peptide. Biomaterials. https://doi.org/10.1016/j.biomaterials.2008.12.018

Liu, X., Yang, J., Wu, N., Song, R., \& Zhu, H. (2015). Evolution and Coevolution of PRC2 Genes in Vertebrates and Mammals. In Advances in Protein Chemistry and Structural Biology. https://doi.org/10.1016/bs.apcsb.2015.06.010

Lopez, A. T., Bates, S., \& Geskin, L. (2018). Current Status of HDAC Inhibitors in Cutaneous T-cell Lymphoma. American Journal of Clinical Dermatology. 
https://doi.org/10.1007/s40257-018-0380-7

Loret, N., Denys, H., Tummers, P., \& Berx, G. (2019). The Role of Epithelial-toMesenchymal Plasticity in Ovarian Cancer Progression and Therapy Resistance. Cancers. https://doi.org/10.3390/cancers11060838

Lou, Y., Preobrazhenska, O., Auf Dem Keller, U., Sutcliffe, M., Barclay, L., McDonald, P. C., ... Dedhar, S. (2008). Epithelial-Mesenchymal Transition (EMT) is not sufficient for spontaneous murine breast cancer metastasis. Developmental Dynamics, 237(10), 2755-2768. https://doi.org/10.1002/dvdy.21658

Lu, W., \& Kang, Y. (2019). Epithelial-Mesenchymal Plasticity in Cancer Progression and Metastasis. Developmental Cell, 49(3), 361-374.

https://doi.org/10.1016/j.devcel.2019.04.010

Luger, K., Mader, A., Richmond, R., Sargent, D., Richmond, T. (1997). Crystal structure of the nucleosome core particle at $2.8 \AA$ resolution. Nature, 389, 251260.

Lukong, K. E. (2017). Understanding breast cancer - The long and winding road. BBA Clinical, 7, 64-77. https://doi.org/10.1016/j.bbacli.2017.01.001

Luo, C., Shaw, K. T., Raghavant, A., Aramburu, J., Cozar, F. G., Perrinoii, B. A., ... Rao, A. (1996). Interaction of calcineurin with a domain of the transcription factor NFAT1 that controls nuclear import. Biochemistry, 93(August), 1-6. Retrieved from papers2://publication/uuid/0B6F2D9A-014E-406F-8A8C-A25AD49871F3

Luqmani, Y. A. (2005). Mechanisms of Drug Resistance in. 14(suppl 1), 35-48. https://doi.org/10.1159/000086183

Ma, X., Ezzeldin, H. H., \& Diasio, R. B. (2009). Histone deacetylase inhibitors: Current status and overview of recent clinical trials. Drugs, 69(14), 1911-1934. https://doi.org/10.2165/11315680-000000000-00000

Macian, F. (2005). NFAT proteins: Key regulators of T-cell development and function. Nature Reviews Immunology, 5(6), 472-484. https://doi.org/10.1038/nri1632

Maenz, C., Lenfert, E., Pantel, K., Schumacher, U., Deppert, W., \& Wegwitz, F. (2015). Epithelial-mesenchymal plasticity is a decisive feature for the metastatic outgrowth of disseminated WAP-T mouse mammary carcinoma cells. BMC Cancer, 15(1), 1-10. https://doi.org/10.1186/s12885-015-1165-5

Mahara, S., Lee, P. L., Feng, M., Tergaonkar, V., Chng, W. J., \& Yu, Q. (2016). HIFI$\alpha$ activation underlies a functional switch in the paradoxical role of Ezh2/PRC2 in breast cancer. Proceedings of the National Academy of Sciences of the United States of America. https://doi.org/10.1073/pnas.1602079113

Mancini, M., \& Toker, A. (2009). NFAT proteins: Emerging roles in cancer progression. Nature Reviews Cancer, 9(11), 810-820.

https://doi.org/10.1038/nrc2735

Mani, S. A., Guo, W., Liao, M. J., Eaton, E. N., Ayyanan, A., Zhou, A. Y., ... Weinberg, R. A. (2008). The Epithelial-Mesenchymal Transition Generates Cells with Properties of Stem Cells. Cell. https://doi.org/10.1016/j.cell.2008.03.027 
Marcato, P., Dean, C. A., Giacomantonio, C. A., \& Lee, P. W. K. (2011). Aldehyde dehydrogenase its role as a cancer stem cell marker comes down to the specific isoform. Cell Cycle. https://doi.org/10.4161/cc.10.9.15486

Margueron, Raphael, Li, G., Sarma, K., Blais, A., Zavadil, J., Woodcock, C. L., ... Reinberg, D. (2008). Ezh1 and Ezh2 Maintain Repressive Chromatin through Different Mechanisms. Molecular Cell. https://doi.org/10.1016/j.molcel.2008.11.004

Margueron, Raphaël, \& Reinberg, D. (2011). The Polycomb complex PRC2 and its mark in life. Nature, 469(7330), 343-349. https://doi.org/10.1038/nature09784

Mariadason, J. M. (2008). Making Sense of HDAC2 Mutations in Colon Cancer. Gastroenterology, 135(5), 1457-1459. https://doi.org/10.1053/j.gastro.2008.09.039

Marks, P. A., \& Breslow, R. (2007). Dimethyl sulfoxide to vorinostat: Development of this histone deacetylase inhibitor as an anticancer drug. Nature Biotechnology, 25(1), 84-90. https://doi.org/10.1038/nbt1272

Martínez-Fernández, M., Dueñas, M., Feber, A., Segovia, C., García-Escudero, R., Rubio, C., ... Paramio, J. M. (2015). A Polycomb-mir200 loop regulates clinical outcome in bladder cancer. Oncotarget, 6(39), 42258-42275. https://doi.org/10.18632/oncotarget.5546

Massihnia, D., Galvano, A., Fanale, D., Perez, A., Castiglia, M., Incorvaia, L., ... Russo, A. (2016). Triple negative breast cancer: shedding light onto the role of pi3k/akt/mtor pathway. Oncotarget, 7(37). https://doi.org/10.18632/oncotarget.10858

Mathiesen, R. R., Fjelldal, R., Liestøl, K., Due, E. U., Geigl, J. B., Riethdorf, S., ... Baumbusch, L. O. (2012). High-resolution analyses of copy number changes in disseminated tumor cells of patients with breast cancer. International Journal of Cancer, 131(4), E405-15. https://doi.org/10.1002/ijc.26444

Matsen, C. B., \& Neumayer, L. A. (2013). Breast cancer: A review for the general surgeon. JAMA Surgery. https://doi.org/10.1001/jamasurg.2013.3393

Mayer, E. L., \& Burstein, H. J. (2007). Chemotherapy for Metastatic Breast Cancer. Hematology/Oncology Clinics of North America. https://doi.org/10.1016/j.hoc.2007.03.001

Méthot, N., \& Basler, K. (2001). An absolute requirement for Cubitus interruptus in Hedgehog signaling. Development. https://doi.org/10.5167/uzh-972

Metzelder, S. K., Michel, C., Von Bonin, M., Rehberger, M., Hessmann, E., Inselmann, S., ... Burchert, A. (2015). NFATc1 as a therapeutic target in FLT3ITD-positive AML. Leukemia. https://doi.org/10.1038/leu.2015.95

Metzger-Filho, O., Sun, Z., Viale, G., Price, K. N., Crivellari, D., Snyder, R. D., ... Cardoso, F. (2013). Patterns of recurrence and outcome according to breast cancer subtypes in lymph node-negative disease: Results from international breast cancer study group trials VIII and IX. Journal of Clinical Oncology, 31(25), 
3083-3090. https://doi.org/10.1200/JCO.2012.46.1574

Min, A., Im, S. A., Kim, D. K., Song, S. H., Kim, H. J., Lee, K. H., ... Bang, Y. J. (2015). Histone deacetylase inhibitor, suberoylanilide hydroxamic acid (SAHA), enhances anti-tumor effects of the poly (ADP-ribose) polymerase (PARP) inhibitor olaparib in triple-negative breast cancer cells. Breast Cancer Research, 17(1), 1-13. https://doi.org/10.1186/s13058-015-0534-y

Mohammad, H. P., Barbash, O., \& Creasy, C. L. (2019). Targeting epigenetic modifications in cancer therapy: erasing the roadmap to cancer. Nature Medicine. https://doi.org/10.1038/s41591-019-0376-8

Morel, A. P., Lièvre, M., Thomas, C., Hinkal, G., Ansieau, S., \& Puisieux, A. (2008). Generation of breast cancer stem cells through epithelial-mesenchymal transition. PLOS ONE. https://doi.org/10.1371/journal.pone.0002888

Morris, G., Naidu, S., Topham, A., Guiles, F., Xu, Y., McCue, P., Schwaartz, G., Park, P., Rosenberg, A., Brill, K., Mitchell, E. (2007). Differences in breast carcinoma characteristics in newly diagnosed African-American and Caucasian patients: a single-institution compilation compared with the National Cancer Institute's Surveillance, Epidemiology, and End Results database. CANCER, Vol. 110, pp. 876-884.

Müller, M. R., \& Rao, A. (2010). NFAT, immunity and cancer: A transcription factor comes of age. Nature Reviews Immunology, 10(9), 645-656.

https://doi.org/10.1038/nri2818

Mullor, J. L., Dahmane, N., Sun, T., \& Altaba, A. R. (2001). Wnt signals are targets and mediators of Gli function. Current Biology. https://doi.org/10.1016/S09609822(01)00229-9

Muto, T., Sashida, G., Oshima, M., Wendt, G. R., Mochizuki-Kashio, M., Nagata, Y., ... Iwama, A. (2013). Concurrent loss of Ezh2 and Tet2 cooperates in the pathogenesis of myelodysplastic disorders. Journal of Experimental Medicine. https://doi.org/10.1084/jem.20131144

Narita, S., So, A., Ettinger, S., Hayashi, N., Muramaki, M., Fazli, L., ... Gleave, M. E. (2008). GLI2 knockdown using an antisense oligonucleotide induces apoptosis and chemosensitizes cells to paclitaxel in androgen-independent Prostate Cancer. Clinical Cancer Research. https://doi.org/10.1158/1078-0432.CCR-074282

Noubissi, F. K., Yedjou, C. G., Spiegelman, V. S., \& Tchounwou, P. B. (2018). Crosstalk between Wnt and $\mathrm{Hh}$ signaling pathways in the pathology of basal cell carcinoma. International Journal of Environmental Research and Public Health. https://doi.org/10.3390/ijerph15071442

O'Reilly, E. A., Gubbins, L., Sharma, S., Tully, R., Guang, M. H. Z., Weiner-Gorzel, K., ... McCann, A. (2015). The fate of chemoresistance in triple negative breast cancer (TNBC). BBA Clinical. https://doi.org/10.1016/j.bbacli.2015.03.003

Oehme, I., Deubzer, H. E., Wegener, D., Pickert, D., Linke, J. P., Hero, B., ... Witt, O. (2009). Histone deacetylase 8 in neuroblastoma tumorigenesis. Clinical Cancer 
Research. https://doi.org/10.1158/1078-0432.CCR-08-0684

Otto, B., Gruner, K., Heinlein, C., Wegwitz, F., Nollau, P., Ylstra, B., ... Tolstonog, G. V. (2013). Low-grade and high-grade mammary carcinomas in WAP-T transgenic mice are independent entities distinguished by Met expression. International Journal of Cancer, 132(6), 1300-1310. https://doi.org/10.1002/ijc.27783

Otto, B., Streichert, T., Wegwitz, F., Gevensleben, H., Klätschke, K., Wagener, C., ... Tolstonog, G. V. (2013). Transcription factors link mouse WAP-T mammary tumors with human breast cancer. International Journal of Cancer, 132(6), 13111322. https://doi.org/10.1002/ijc.27941

Pan, M.-G., Xiong, Y., \& Chen, F. (2013). NFAT Gene Family in Inflammation and Cancer. Current Molecular Medicine. https://doi.org/10.2174/1566524011313040007

Parsons, X. (2014). Embedding the Future of Regenerative Medicine into the Open Epigenomic. Annual Research \& Review in Biology, 3(4), 323-349.

Pasini, D., Bracken, A. P., Jensen, M. R., Denchi, E. L., \& Helin, K. (2004). Suz12 is essential for mouse development and for $\mathrm{EZH} 2$ histone methyltransferase activity. EMBO Journal. https://doi.org/10.1038/sj.emboj.7600402

Paznekas, W. A., Okajima, K., Schertzer, M., Wood, S., \& Jabs, E. W. (1999). Genomic organization, expression, and chromosome location of the human SNAIL gene (SNAI1) and a related processed pseudogene (SNAI1P). Genomics. https://doi.org/10.1006/geno.1999.6010

Perou, C. M., Sørile, T., Eisen, M. B., Van De Rijn, M., Jeffrey, S. S., Ress, C. A., ... Botstein, D. (2000). Molecular portraits of human breast tumours. Nature. https://doi.org/10.1038/35021093

Pham, L. V., Tamayo, A. T., Li, C., Bueso-Ramos, C., \& Ford, R. J. (2010). An epigenetic chromatin remodeling role for NFATc1 in transcriptional regulation of growth and survival genes in diffuse large B-cell lymphomas. Blood. https://doi.org/10.1182/blood-2009-12-257378

Pineda, B., Diaz-Lagares, A., Pérez-Fidalgo, J. A., Burgués, O., González-Barrallo, I., Crujeiras, A. B., ... Eroles, P. (2019). A two-gene epigenetic signature for the prediction of response to neoadjuvant chemotherapy in triple-negative breast cancer patients. Clinical Epigenetics. https://doi.org/10.1186/s13148-019-0626-0

Prakriya, M., Feske, S., Gwack, Y., Srikanth, S., Rao, A., \& Hogan, P. G. (2006). Orai1 is an essential pore subunit of the CRAC channel. Nature, 443(7108), 230233. https://doi.org/10.1038/nature05122

Prasad, A. M., \& Inesi, G. (2009). Effects of thapsigargin and phenylephrine on calcineurin and protein kinase $\mathrm{C}$ signaling functions in cardiac myocytes. American Journal of Physiology-Cell Physiology. https://doi.org/10.1152/ajpcell.00594.2008

Prat, A., Fan, C., Fernández, A., Hoadley, K. A., Martinello, R., Vidal, M., ... Perou, C. M. (2015). Response and survival of breast cancer intrinsic subtypes following 
multi-agent neoadjuvant chemotherapy. BMC Medicine, 13(1), 1-11. https://doi.org/10.1186/s12916-015-0540-z

Prat, A., Pineda, E., Adamo, B., Galván, P., Fernández, A., Gaba, L., ... Muñoz, M. (2015). Clinical implications of the intrinsic molecular subtypes of breast cancer. Breast, 24, S26-S35. https://doi.org/10.1016/j.breast.2015.07.008

Qin, J. J., Nag, S., Wang, W., Zhou, J., Zhang, W. D., Wang, H., \& Zhang, R. (2014). NFAT as cancer target: Mission possible? Biochimica et Biophysica Acta Reviews on Cancer. https://doi.org/10.1016/j.bbcan.2014.07.009

Raman, J. D., Mongan, N. P., Tickoo, S. K., Boorjian, S. A., Scherr, D. S., \& Gudas, L. J. (2005). Increased expression of the polycomb group gene, EZH2, in transitional cell carcinoma of the bladder. Clinical Cancer Research. https://doi.org/10.1158/1078-0432.CCR-05-1047

Rao, A., Luo, C., \& Hogan, P. G. (1997). TRANSCRIPTION FACTORS OF THE NFAT FAMILY:Regulation and Function. Annual Review of Immunology, 15(1), 707-747. https://doi.org/10.1146/annurev.immunol.15.1.707

Reik, W. (2007). Stability and flexibility of epigenetic gene regulation in mammalian development. Nature. https://doi.org/10.1038/nature05918

Rettig, I., Koeneke, E., Trippel, F., Mueller, W. C., Burhenne, J., Kopp-Schneider, A., ... Oehme, I. (2015). Selective inhibition of HDAC8 decreases neuroblastoma growth in vitro and in vivo and enhances retinoic acid-mediated differentiation. Cell Death and Disease. https://doi.org/10.1038/cddis.2015.24

Richon, V. M., Sandhoff, T. W., Rifkind, R. A., \& Marks, P. A. (2000). Histone deacetylase inhibitor selectively induces p21WAF1 expressjon and geneassociated histone acetylation. Proceedings of the National Academy of Sciences of the United States of America. https://doi.org/10.1073/pnas.180316197

Robbs, B. K., Cruz, A. L. S., Werneck, M. B. F., Mognol, G. P., \& Viola, J. P. B. (2008). Dual Roles for NFAT Transcription Factor Genes as Oncogenes and Tumor Suppressors. Molecular and Cellular Biology, 28(23), 7168-7181. https://doi.org/10.1128/MCB.00256-08

Robson, M. (2011). Poly(ADP-ribose) polymerase inhibitors in triple-negative breast cancer. In Advances in the Management of Triple-Negative Breast Cancer. https://doi.org/10.2217/EBO.11.100

Rosato, R. R. (2005). The Histone Deacetylase Inhibitor LAQ-824 Induces Human Leukemia Cell Death through a Process Involving XIAP Down-Regulation, Oxidative Injury, and the Acid Sphingomyelinase-Dependent Generation of Ceramide. Molecular Pharmacology, 69(1), 216-225. https://doi.org/10.1124/mol.105.017145

Roth, S. Y., Denu, J. M., \& Allis, C. D. (2001). Histone Acetyltransferases. Annual Review of Biochemistry. https://doi.org/10.1146/annurev.biochem.70.1.81

Rottenberg, S., Jaspers, J. E., Kersbergen, A., van der Burg, E., Nygren, A. O. H., 
Zander, S. A. L., ... Jonkers, J. (2008). High sensitivity of BRCA1-deficient mammary tumors to the PARP inhibitor AZD2281 alone and in combination with platinum drugs. Proceedings of the National Academy of Sciences. https://doi.org/10.1073/pnas.0806092105

Sakamoto, K. M., \& Aldana-Masangkay, G. I. (2011). The role of HDAC6 in cancer. Journal of Biomedicine and Biotechnology. https://doi.org/10.1155/2011/875824

Sakamoto, T., Kobayashi, S., Yamada, D., Nagano, H., Tomokuni, A., Tomimaru, Y., ... Mori, M. (2016). A Histone deacetylase inhibitor suppresses epithelialmesenchymal transition and attenuates chemoresistance in biliary tract cancer. PLOS ONE, 11(1). https://doi.org/10.1371/journal.pone.0145985

San-Miguel, J. F., Richardson, P. G., Günther, A., Sezer, O., Siegel, D., Bladé, J., ... Anderson, K. C. (2013). Phase lb study of panobinostat and bortezomib in relapsed or relapsed and refractory multiple myeloma. Journal of Clinical Oncology. https://doi.org/10.1200/JCO.2012.46.7068

Saotome, K., Morita, H., \& Umeda, M. (1989). Cytotoxicity test with simplified crystal violet staining method using microtitre plates and its application to injection drugs. Toxicology in Vitro. https://doi.org/10.1016/0887-2333(89)90039-8

Scheel, C., Eaton, E. N., Li, S. H. J., Chaffer, C. L., Reinhardt, F., Kah, K. J., ... Weinberg, R. A. (2011). Paracrine and autocrine signals induce and maintain mesenchymal and stem cell states in the breast. Cell. https://doi.org/10.1016/j.cell.2011.04.029

Scheel, C., \& Weinberg, R. A. (2011). Phenotypic plasticity and epithelialmesenchymal transitions in cancer and normal stem cells? International Journal of Cancer. https://doi.org/10.1002/ijc.26311

Schulze-Garg, C., Löhler, J., Gocht, A., \& Deppert, W. (2000). A transgenic mouse model for the ductal carcinoma in situ (DCIS) of the mammary gland. Oncogene. https://doi.org/10.1038/sj.onc.1203281

Sengupta, S., Jana, S., Biswas, S., Mandal, P. K., \& Bhattacharyya, A. (2013). Cooperative involvement of NFAT and SnoN mediates transforming growth factor- $\beta$ (TGF- $\beta$ ) induced EMT in metastatic breast cancer (MDA-MB 231) cells. Clinical and Experimental Metastasis. https://doi.org/10.1007/s10585-013-9600-y

Serresi, M., Gargiulo, G., Proost, N., Siteur, B., Cesaroni, M., Koppens, M., ... van Lohuizen, M. (2016). Polycomb Repressive Complex 2 Is a Barrier to KRASDriven Inflammation and Epithelial-Mesenchymal Transition in Non-Small-Cell Lung Cancer. Cancer Cell. https://doi.org/10.1016/j.ccell.2015.12.006

Seto, E., \& Yoshida, M. (2014). Erasers of histone acetylation: The histone deacetylase enzymes. Cold Spring Harbor Perspectives in Biology. https://doi.org/10.1101/cshperspect.a018713

Shackleton, M., Quintana, E., Fearon, E. R., \& Morrison, S. J. (2009). Heterogeneity in Cancer: Cancer Stem Cells versus Clonal Evolution. Cell. https://doi.org/10.1016/j.cell.2009.08.017 
Shan, W., Jiang, Y., Yu, H., Huang, Q., Liu, L., Guo, X., ... Yang, Z. (2017). HDAC2 overexpression correlates with aggressive clinicopathological features and DNAdamage response pathway of breast cancer. American Journal of Cancer Research.

Shaw, J. P., Utz, P. J., Durand, D. B., Toole, J. J., Emmel, E. A., \& Crabtree, G. R. (1988). Identification of a putative regulator of early T cell activation genes. Journal of Immunology, 185(9), 4972-4975.

https://doi.org/10.1126/science.3260404

Shaw, J. P., Utz, P. J., Durand, D. B., Toole, J. J., Emmel, E. A., \& Crabtree, G. R. (2010). Identification of a putative regulator of early $T$ cell activation genes. Journal of Immunology, 185(9), 4972-4975. https://doi.org/10.1126/science.3260404

Shi, B., Liang, J., Yang, X., Wang, Y., Zhao, Y., Wu, H., ... Shang, Y. (2007). Integration of Estrogen and Wnt Signaling Circuits by the Polycomb Group Protein EZH2 in Breast Cancer Cells. Molecular and Cellular Biology. https://doi.org/10.1128/mcb.00162-07

Shibue, T., \& Weinberg, R. A. (2017). EMT, CSCs, and drug resistance: The mechanistic link and clinical implications. Nature Reviews Clinical Oncology. https://doi.org/10.1038/nrclinonc.2017.44

Sihto, H., Lundin, J., Lundin, M., Lehtimäki, T., Ristimäki, A., Holli, K., ... Joensuu, H. (2011). Breast cancer biological subtypes and protein expression predict for the preferential distant metastasis sites: A nationwide cohort study. Breast Cancer Research, 13(5), R87. https://doi.org/10.1186/bcr2944

Sikov, W. M., Berry, D. A., Perou, C. M., Singh, B., Cirrincione, C. T., Tolaney, S. M., ... Winer, E. P. (2015). Impact of the addition of carboplatin and/or bevacizumab to neoadjuvant once-per-week paclitaxel followed by dose-dense doxorubicin and cyclophosphamide on pathologic complete response rates in stage II to III triple-negative breast cancer: CALGB 40603 (A. Journal of Clinical Oncology. https://doi.org/10.1200/JCO.2014.57.0572

Simõ-Riudalbas, L., \& Esteller, M. (2015). Targeting the histone orthography of cancer: Drugs for writers, erasers and readers. British Journal of Pharmacology. https://doi.org/10.1111/bph.12844

Simon, J. A., \& Kingston, R. E. (2013). Occupying Chromatin: Polycomb Mechanisms for Getting to Genomic Targets, Stopping Transcriptional Traffic, and Staying Put. Molecular Cell. https://doi.org/10.1016/j.molcel.2013.02.013

Singh, A. K., Bishayee, A., \& Pandey, A. K. (2018). Targeting histone deacetylases with natural and synthetic agents: An emerging anticancer strategy. Nutrients. https://doi.org/10.3390/nu10060731

Sirohi, B., Arnedos, M., Popat, S., Ashley, S., Nerurkar, A., Walsh, G., ... Smith, I. E. (2008). Platinum-based chemotherapy in triple-negative breast cancer. Annals of Oncology. https://doi.org/10.1093/annonc/mdn395

Skrypek, N., Goossens, S., De Smedt, E., Vandamme, N., \& Berx, G. (2017). 
Epithelial-to-Mesenchymal Transition: Epigenetic Reprogramming Driving Cellular Plasticity. Trends in Genetics, 33(12), 943-959.

https://doi.org/10.1016/j.tig.2017.08.004

Slingerland, M., Guchelaar, H. J., \& Gelderblom, H. (2014). Histone deacetylase inhibitors: An overview of the clinical studies in solid tumors. Anti-Cancer Drugs. https://doi.org/10.1097/CAD.0000000000000040

Smith, B., \& Bhowmick, N. (2016). Role of EMT in Metastasis and Therapy Resistance. Journal of Clinical Medicine. https://doi.org/10.3390/jcm5020017

Solomon, E. I., Augustine, A. J., \& Yoon, J. (2015). A class I histone deacetylase inhibitor, entinostat, enhances lapatinib efficacy in HER2-overexpressing breast cancer cells through FOXO3-mediated Bim1 expression. Breast Cancer Research and Treatment, 146(30), 259-272. https://doi.org/10.1007/s10549-0143014-7

Song, X., Gao, T., Wang, N., Feng, Q., You, X., Ye, T., ... Yu, L. (2016). Selective inhibition of EZH2 by ZLD1039 blocks H3K27methylation and leads to potent anti-tumor activity in breast cancer. Scientific Reports, 6(January), 1-13. https://doi.org/10.1038/srep20864

Stahl, M., Kohrman, N., Gore, S. D., Kim, T. K., Zeidan, A. M., \& Prebet, T. (2016). Epigenetics in Cancer: A Hematological Perspective. PLOS Genetics. https://doi.org/10.1371/journal.pgen.1006193

Stark, R., \& Brown, G. (2011). DiffBind : differential binding analysis of ChIP-Seq peak data. Bioconductor.

Stiff, J., \& Marrow, B. (1995). High-Dose Cyclosporin for the A Phase Chemotherapy $A$ and I Clinical Treatment Combined with Bone Solid Escalating Marrow Tumors : Doses Transplant of an Autologous of Drug-resistant Trial. 1(December), 1495-1502.

Su, Y., Hopfinger, N. R., Nguyen, T. D., Pogash, T. J., Santucci-Pereira, J., \& Russo, J. (2018). Epigenetic reprogramming of epithelial mesenchymal transition in triple negative breast cancer cells with DNA methyltransferase and histone deacetylase inhibitors. Journal of Experimental and Clinical Cancer Research. https://doi.org/10.1186/s13046-018-0988-8

Subramanian, S., Bates, S. E., Wright, J. J., Espinoza-Delgado, I., \& Piekarz, R. L. (2010). Clinical toxicities of histone deacetylase inhibitors. Pharmaceuticals. https://doi.org/10.3390/ph3092751

Takebe, N., Miele, L., Harris, P. J., Jeong, W., Bando, H., Kahn, M., ... Ivy, S. P. (2015). Targeting Notch, Hedgehog, and Wnt pathways in cancer stem cells: Clinical update. Nature Reviews Clinical Oncology, 12(8), 445-464. https://doi.org/10.1038/nrclinonc.2015.61

Teng, Y., Bai, F., Elankumaran, S., Li, R., Zhang, H., Xing, J., ... Zhang, J. (2014). TGF- -induced epithelial-to-mesenchymal transition proceeds through stepwise activation of multiple feedback loops. Science Signaling, 7(345), 1-12. https://doi.org/10.1126/scisignal.2005304 
Thiery, J. P. (2002). Epithelial-mesenchymal transitions in tumour progression. Nature Reviews Cancer, 2(6), 442-454. https://doi.org/10.1038/nrc822

Tie, F., Banerjee, R., Stratton, C. A., Prasad-Sinha, J., Stepanik, V., Zlobin, A., ... Harte, P. J. (2009). CBP-mediated acetylation of histone H3 lysine 27 antagonizes Drosophila Polycomb silencing. Development. https://doi.org/10.1242/dev.037127

Toft, D. J., \& Cryns, V. L. (2010). Minireview: Basal-Like Breast Cancer: From Molecular Profiles to Targeted Therapies. Molecular Endocrinology, 25(2), 199211. https://doi.org/10.1210/me.2010-0164

Toker, A., Yoeli-Lerner, M., Chin, Y. R., \& Hansen, C. K. (2009). The AKT/PKB and GSK-3 $\beta$ Signaling Pathway regulates Cell Migration through the Nfat1 Transcription Factor. Molecular Cancer Research, 7(3), 425-432. https://doi.org/10.1158/1541-7786.MCR-08-0342.THE

Towbin, H., Staehelin, T., \& Gordon, J. (1979). Electrophoretic transfer of proteins from polyacrylamide gels to nitrocellulose sheets: procedure and some applications. Proceedings of the National Academy of Sciences. https://doi.org/10.1073/pnas.76.9.4350

Tran Quang, C., Leboucher, S., Passaro, D., Fuhrmann, L., Nourieh, M., VincentSalomon, A., \& Ghysdael, J. (2015). The calcineurin/NFAT pathway is activated in diagnostic breast cancer cases and is essential to survival and metastasis of mammary cancer cells. Cell Death and Disease, 6(2), e1658-10. https://doi.org/10.1038/cddis.2015.14

Trivers, K. F., Lund, M. J., Porter, P. L., Liff, J. M., Flagg, E. W., Coates, R. J., \& Eley, J. W. (2009). The epidemiology of triple-negative breast cancer, including race. Cancer Causes and Control. https://doi.org/10.1007/s10552-009-9331-1

Vaeth, M., \& Feske, S. (2018). NFAT control of immune function: New Frontiers for an Abiding Trooper. F1000Research, 7(0), 260. https://doi.org/10.12688/f1000research.13426.1

Vallejos, C., Gómez, H., Cruz, W., Pinto, J., Dyer, R., Velarde, R., ... Vigil, C. (2010). Breast cancer classification according to immunohistochemistry markers:

Subtypes and association with clinicopathologic variables in a peruvian hospital database. Clinical Breast Cancer, 10(4), 294-300.

https://doi.org/10.3816/CBC.2010.n.038

Varambally, S., Dhanasekaran, S. M., Zhou, M., Barrette, T. R., Kumar-Sinha, C., Sanda, M. G., ... Chinnaiyan, A. M. (2002). The polycomb group protein EZH2 is involved in progression of prostate cancer. Nature.

https://doi.org/10.1038/nature01075

Veronesi, U., Cascinelli, N., Mariani, L., Greco, M., Saccozzi, R., Luini, A., ... Marubini, E. (2002). Twenty-year follow-up of a randomized study comparing breast-conserving surgery with radical mastectomy for early breast cancer. The New England Journal of Medicine, 347(16), 1227-1232. https://doi.org/10.1056/NEJMoa020989 
Ververis, K., Hiong, A., Karagiannis, T. C., \& Licciardi, P. V. (2013). Histone deacetylase inhibitors (HDACls): multitargeted anticancer agents. Biologics:

Targets and Therapy, 7, 47-60. https://doi.org/10.2147/BTT.S29965

Vizán, P., Beringer, M., Ballaré, C., \& Di Croce, L. (2015). Role of PRC2-associated factors in stem cells and disease. The FEBS Journal, 282(9), 1723-1735. https://doi.org/10.1111/febs.13083

Vo, B. H. T., Li, C., Morgan, M. A., Theurillat, I., Finkelstein, D., Wright, S., ... Roussel, M. F. (2017). Inactivation of Ezh2 Upregulates Gfi1 and Drives Aggressive Myc-Driven Group 3 Medulloblastoma. Cell Reports. https://doi.org/10.1016/j.celrep.2017.02.073

Vogelauer, M., Krall, A. S., McBrian, M. A., Li, J. Y., \& Kurdistani, S. K. (2012). Stimulation of histone deacetylase activity by metabolites of intermediary metabolism. Journal of Biological Chemistry, 287(38), 32006-32016. https://doi.org/10.1074/jbc.M112.362467

Völkel, P., Dupret, B., Le Bourhis, X., \& Angrand, P. O. (2015). Diverse involvement of EZH2 in cancer epigenetics. American Journal of Translational Research.

Waddington, C. H. (1957). The strategy of the genes. A discussion of some aspects of theoretical biology. With an appendix by H. Kacser. The Strategy of the Genes A Discussion of Some .... https://doi.org/10.1007/3-540-32786-X_7

Wahba, H. A., \& El-Hadaad, H. A. (2015). Current approaches in treatment of triplenegative breast cancer. Cancer Biology and Medicine. https://doi.org/10.7497/j.issn.2095-3941.2015.0030

Wainwright, E. N., \& Scaffidi, P. (2017). Epigenetics and Cancer Stem Cells: Unleashing, Hijacking, and Restricting Cellular Plasticity. Trends in Cancer, 3(5), 372-386. https://doi.org/10.1016/j.trecan.2017.04.004

Wang, Z. C., Birkbak, N. J., Culhane, A. C., Drapkin, R., Fatima, A., Tian, R., ... Matulonis, U. A. (2012). Profiles of genomic instability in high-grade serous ovarian cancer predict treatment outcome. Clinical Cancer Research. https://doi.org/10.1158/1078-0432.CCR-12-0857

Wassef, M., Rodilla, V., Teissandier, A., Zeitouni, B., Gruel, N., Sadacca, B., ... Margueron, R. (2015). Impaired PRC2 activity promotes transcriptional instability and favors breast tumorigenesis. Genes and Development. https://doi.org/10.1101/gad.269522.115

Wathieu, H., Issa, N. T., Fernandez, A. I., Mohandoss, M., Tiek, D. M., Franke, J. L., ... Dakshanamurthy, S. (2017). Differential prioritization of therapies to subtypes of triple negative breast cancer using a systems medicine method. Oncotarget, 8(54). https://doi.org/10.18632/oncotarget.21669

Wegwitz, F., Kluth, M. A., Mänz, C., Otto, B., Gruner, K., Heinlein, C., ... Tolstonog, G. V. (2010). Tumorigenic WAP-T mouse mammary carcinoma cells: A model for a self-reproducing homeostatic cancer cell system. PLOS ONE. https://doi.org/10.1371/journal.pone.0012103 
Weichert, W., Röske, A., Gekeler, V., Beckers, T., Stephan, C., Jung, K., ... Kristiansen, G. (2008). Histone deacetylases 1, 2 and 3 are highly expressed in prostate cancer and HDAC2 expression is associated with shorter PSA relapse time after radical prostatectomy. British Journal of Cancer, 98(3), 604-610. https://doi.org/10.1038/sj.bjc.6604199

Wen, Y., Cai, J., Hou, Y., Huang, Z., \& Wang, Z. (2017). Role of EZH2 in cancer stem cells: from biological insight to a therapeutic target. Oncotarget, 8(23), 3797437990. https://doi.org/10.18632/oncotarget.16467

Wolf, C., Filarsky, K., Garding, A., Claus, R., Oakes, C., Idler, I., ... Mertens, D. (2014). NFATc1 Is Transcriptionally Activated in Chronic Lymphocytic Leukemia (CLL) By Promotor DNA-Hypomethylation Which Correlates with in-Vitro Vulnerability to Calcineurin Inhibitors. Blood, 124(21).

Wong, Eric, Chaudhry, Sultan, Rossi, M. (2012). BREAST CANCER PATHOGENESIS AND HISTOLOGIC VS. MOLECULAR SUBTYPES. Retrieved from http://www.pathophys.org/breast-cancer/breastcancer-copy/

World Health Organisation. (2018). Global cancer data. International Agency for Research on Cancer, (September), 13-15. Retrieved from http://gco.iarc.fr/,

World Health Organization. (2006). Guidelines for managements of breast cancer. In World Health Organization.

Wouters, B. J., \& Delwel, R. (2016). Epigenetics and approaches to targeted epigenetic therapy in acute myeloid leukemia. Blood. https://doi.org/10.1182/blood-2015-07-604512

Xu, K., Wu, Z. J., Groner, A. C., He, H. H., Cai, C., Lis, R. T., ... Brown, M. (2012). EZH2 Oncogenic Activity in Castration-Resistant Prostate Cancer Cells Is Polycomb-Independent. Science. https://doi.org/10.1126/science.1227604

Ye, X., Brabletz, T., Kang, Y., Longmore, G. D., Nieto, M. A., Stanger, B. Z., ... Weinberg, R. A. (2017). Upholding a role for EMT in breast cancer metastasis. Nature. https://doi.org/10.1038/nature22816

Ye, X., \& Weinberg, R. A. (2015). Epithelial-Mesenchymal Plasticity: A Central Regulator of Cancer Progression. Trends in Cell Biology. https://doi.org/10.1016/j.tcb.2015.07.012

Young, M. D., Willson, T. A., Wakefield, M. J., Trounson, E., Hilton, D. J., Blewitt, M. E., ... Majewski, I. J. (2011). ChIP-seq analysis reveals distinct H3K27me3 profiles that correlate with transcriptional activity. Nucleic Acids Research. https://doi.org/10.1093/nar/gkr416

Yu, H., Van Berkel, T. J. C., \& Biessen, E. A. L. (2007). Therapeutic potential of VIVIT, a selective peptide inhibitor of nuclear factor of activated T cells, in cardiovascular disorders. Cardiovascular Drug Reviews. https://doi.org/10.1111/j.1527-3466.2007.00011.x

Zeng, L. S., Yang, X. Z., Wen, Y. F., Mai, S. J., Wang, M. H., Zhang, M. Y., ... Wang, H. Y. (2016). Overexpressed HDAC4 is associated with poor survival and 
promotes tumor progression in esophageal carcinoma. Aging.

https://doi.org/10.18632/aging.100980

Zhang, P., Xiao, Z., Wang, S., Zhang, M., Wei, Y., Hang, Q., ... Ma, L. (2018). ZRANB1 Is an EZH2 Deubiquitinase and a Potential Therapeutic Target in Breast Cancer. Cell Reports. https://doi.org/10.1016/j.celrep.2018.03.078

Zhang, Yi, Liu, G., Lin, C., Liao, G., \& Tang, B. (2013). Silencing the EZH2 gene by RNA interference reverses the drug resistance of human hepatic multidrugresistant cancer cells to 5-Fu. Life Sciences. https://doi.org/10.1016/j.lfs.2013.03.010

Zhang, Yong, Liu, T., Meyer, C. A., Eeckhoute, J., Johnson, D. S., Bernstein, B. E., ... Shirley, X. S. (2008). Model-based analysis of ChIP-Seq (MACS). Genome Biology. https://doi.org/10.1186/gb-2008-9-9-r137

Zhao, J. H., Luo, Y., Jiang, Y. G., He, D. L., \& Wu, C. T. (2011). Knockdown of $\beta$ catenin through shRNA cause a reversal of EMT and metastatic phenotypes induced by HIF-1a. Cancer Investigation, 29(6), 377-382. https://doi.org/10.3109/07357907.2010.512595

Zhou, H., Zhang, Z., Liu, C., Jin, C., Zhang, J., Miao, X., \& Jia, L. (2012). B4GALT1 gene knockdown inhibits the hedgehog pathway and reverses multidrug resistance in the human leukemia K562/adriamycin-resistant cell line. IUBMB Life. https://doi.org/10.1002/iub.1080

Zhu, C., Chen, Q., Xie, Z., Ai, J., Tong, L., Ding, J., \& Geng, M. (2011). The role of histone deacetylase 7 (HDAC7) in cancer cell proliferation: Regulation on c-Myc. Journal of Molecular Medicine. https://doi.org/10.1007/s00109-010-0701-7 